\title{
Frantz FANON
}

Psychiatre et militant de l'indépendance algérienne dans le FLN

\section{(2001)}

\section{Pour la révolution africaine Écrits politiques}

Un document produit en version numérique par Émilie Tremblay, bénévole, Doctorante en sociologie à l'Université de Montréal Courriel: emiliet82@yahoo.fr

Page web dans Les Classiques des sciences sociales.

Dans le cadre de: "Les classiques des sciences sociales" Une bibliothèque numérique fondée et dirigée par Jean-Marie Tremblay, professeur de sociologie au Cégep de Chicoutimi

Site web: http://classiques.uqac.ca/

Une collection développée en collaboration avec la Bibliothèque Paul-Émile-Boulet de l'Université du Québec à Chicoutimi Site web: http://bibliotheque.uqac.ca/ 


\section{Politique d'utilisation de la bibliothèque des Classiques}

Toute reproduction et rediffusion de nos fichiers est interdite, même avec la mention de leur provenance, sans l'autorisation formelle, écrite, du fondateur des Classiques des sciences sociales, Jean-Marie Tremblay, sociologue.

Les fichiers des Classiques des sciences sociales ne peuvent sans autorisation formelle:

- être hébergés (en fichier ou page web, en totalité ou en partie) sur un serveur autre que celui des Classiques.

- servir de base de travail à un autre fichier modifié ensuite par tout autre moyen (couleur, police, mise en page, extraits, support, etc...),

Les fichiers (.html, .doc, .pdf, .rtf, .jpg, .gif) disponibles sur le site Les Classiques des sciences sociales sont la propriété des Classiques des sciences sociales, un organisme à but non lucratif composé exclusivement de bénévoles.

Ils sont disponibles pour une utilisation intellectuelle et personnelle et, en aucun cas, commerciale. Toute utilisation à des fins commerciales des fichiers sur ce site est strictement interdite et toute rediffusion est également strictement interdite.

L'accès à notre travail est libre et gratuit à tous les utilisateurs. C'est notre mission.

Jean-Marie Tremblay, sociologue

Fondateur et Président-directeur général, LES CLASSIQUES DES SCIENCES SOCIALES. 


\section{REMARQUE}

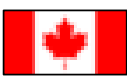

Ce livre est du domaine public au Canada parce qu'une œuvre passe au domaine public 50 ans après la mort de l'auteur(e).

Cette œuvre n'est pas dans le domaine public dans les pays où il faut attendre 70 ans après la mort de l'auteur(e).

Respectez la loi des droits d'auteur de votre pays. 
Cette édition électronique a été réalisée par Émilie Tremblay, bénévole, doctorante en sociologie à l'Université de Montréal

Courriel : emiliet82@yahoo.fr

à partir de :

Frantz FANON

Pour la révolution africaine.

Écrits politiques.

Paris: Éditions La Découverte, 2001, 228 pp. Collection $[R e]$ découverte, documents et témoignages.

Polices de caractères utilisée:

Pour le texte: Comic Sans, 12 points.

Pour les citations : Comic Sans, 12 points.

Pour les notes de bas de page : Comic Sans, 10 points.

Édition électronique réalisée avec le traitement de textes Microsoft Word 2008 pour Macintosh.

Mise en page sur papier format : LETTRE US, $8.5^{\prime \prime} \times 11^{\prime \prime}$ )

Édition numérique réalisée le 6 décembre 2011 à Chicoutimi, Ville de Saguenay, Québec.

\section{E Fait avec}

Macintosh 


\section{Frantz FANON}

Psychiatre, intellectuel antillais

et militant de l'indépendance algérienne dans le FLN

\section{Pour la révolution africaine. Écrits politiques.}

Frantz Fanon

Pour la révolution africaine

Écrits politiques

2: Une reflexion sur le pbinomène colonial, oficu de l'imte-

rieur s: Des texter qui denoncent à la fois le colonialisme ef

les prieges de la décolonisation - la a grande erreur

blamche s et le a grand minage noir * \$t Le colonisé en

question * Racisme et culture * Pour l'Algérie t:

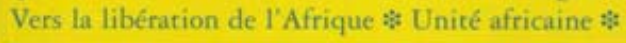

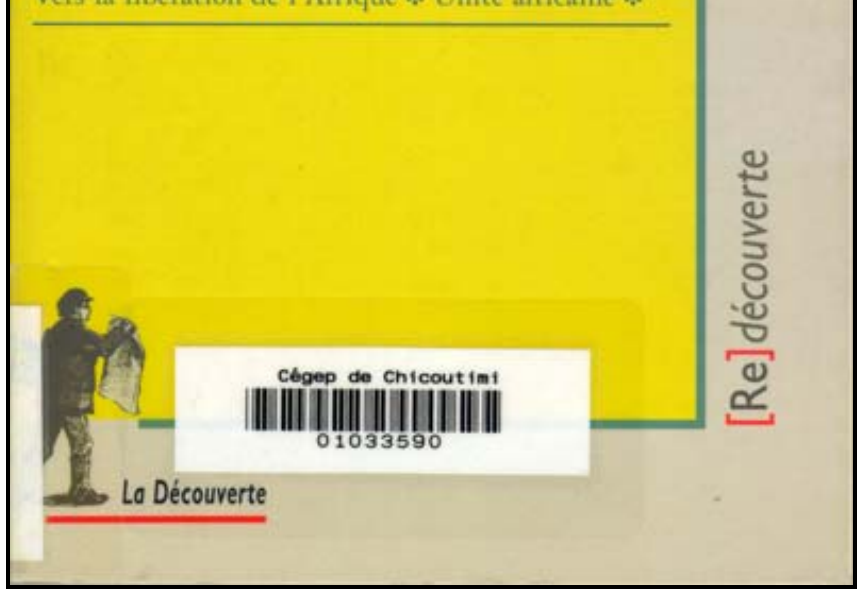

Paris: Éditions La Découverte, 2001, 228 pp. Collection $[R e]$ découverte, documents et témoignages. 
[6]

Cet ouvrage a été précédemment publié en 1964 dans la collection «Cahiers libres» (Maspero) et réédité dans la «Petite collection Maspero» en 1969. 


\section{Table des matières}

Quatrième de couverture

Note de l'éditeur

\section{Le colonisé en question}

1. Le "syndrome nord-africain»

2. Antillais et Africains

II. Racisme et culture

\section{Pour l'Algérie}

1. Lettre à un Français

2. Lettre au Ministre-Résident

IV. Vers la libération de l'Afrique

1. Déceptions et illusions du colonialisme français

2. L'Algérie face aux tortionnaires français

3. À propos d'un plaidoyer

4. Les intellectuels et les démocrates français devant la Révolution Algérienne

5. Aux Antilles, naissance d'une nation?

6. Le sang maghrébin ne coulera pas en vain

7. La farce qui change de camp

8. Décolonisation et indépendance

9. Une crise continuée

10. Lettre à la jeunesse africaine

11. Vérités premières à propos du problème colonial

12. La leçon de Cotonou

13. Appel aux Africains 
14. Lendemains d'un plébiscite en Afrique

15. La guerre d'Algérie et la libération des hommes

16. L'Algérie à Accra

17. Accra: l'Afrique affirme son unité et définit sa stratégie

18. Les tentatives désespérées de M. Debré

19. Fureur raciste en France

20. Le sang coule aux Antilles sous domination française

21. Unité et solidarité effective sont les conditions de la libération africaine

V. Unité africaine

1. Cette Afrique à venir

2. La mort de Lumumba : Pouvions-nous faire autrement 


\section{Pour la révolution africaine}

Écrits politiques

\section{QUATRIÈME DE COUVERTURE}

\section{Retour à la table des matières}

Les textes politiques de Frantz Fanon publiés dans ce volume couvrent la période la plus active de sa vie, de la publication de Peau noire, masques blancs en 1952 - il avait alors vingt-huit ans - à celle des Damnés de la terre en 1961 qui devait coïncider, à quelques jours près, avec la date de sa mort. Retraçant le fil d'une réflexion en constante évolution sur le phénomène colonial, vécu de l'intérieur, ces textes dénoncent à la fois le colonialisme et les pièges de la décolonisation, - la « grande erreur blanche » et le « grand mirage noir ».

Explorant tour à tour la situation du colonisé, dont il peut rendre compte scientifiquement par son expérience médicale quotidienne, l'attitude des intellectuels de gauche face à la guerre d'Algérie, les perspectives de conjonction de la lutte de tous les colonisés et les conditions d'une alliance de l'ensemble du continent africain, Frantz Fanon gardait la certitude de la prochaine libération totale de l'Afrique. Son analyse et la clarté de sa vision nous donnent aujourd'hui les clés nécessaires pour comprendre la réalité africaine actuelle.

Né antillais, mort algérien à l'âge de 36 ans, psychiatre, militant FLN, Frantz Fanon (1925-1961) est notamment l'auteur de Peau noire, masques blancs, L'an $V$ de la révolution algérienne et Les damnés de la terre. 
Cet ouvrage a été précédemment publié en 1964 dans la collection « Cahiers libres» (Maspero) et réédité dans la «Petite collection Maspero » en 1969. 
Pour la révolution africaine Écrits politiques

\section{NOTE DE L'ÉDITEUR}

\section{Retour à la table des matières}

Les textes politiques de Frantz Fanon publiés dans ce volume couvrent la période la plus active de sa vie, de la publication de Peau noire, Masques blancs en 1952 - il avait alors vingt-huit ans - à celle des Damnés de la terre en 1961 qui devait coïncider à quelques jours près avec la date de sa mort.

La plupart de ces textes ne sont pas inédits. Ils ont été publiés dans diverses revues et périodiques dont nous donnons à chaque fois la référence et la date. Mais ils y restaient épars et difficiles à retrouver. Ceux d'El Moudjahid, en particulier, ne sont guère accessibles aujourd'hui, et ne l'ont été, à l'époque même, que pour une partie limitée du public.

Regroupés ainsi dans l'ordre chronologique, ces textes font jaillir une unité singulièrement vivante. Ils marquent les étapes successives d'un même combat, qui évolue et s'élargit, mais dont l'objectif et les moyens ont été vus et fixés depuis le début. Les trois livres publiés jusqu'ici nous donnaient trois analyses cristallisées à des moments précis de l'évolution de F. Fanon. Les textes qui suivent sont un fil conducteur plus quotidien, l'itinéraire d'une pensée en perpétuelle évo- 
lution, qui va constamment s'élargissant et s'enrichissant, tout en restant toujours fidèle à elle-même.

Les deux premiers articles, Le syndrome Nord-Africain et Antillais et Africains publiés en 1952 et 1955 peuvent marquer les premières étapes. A ce moment, Frantz Fanon a terminé ses études de psychiatrie: il peut ainsi, d'une part rendre [8] compte scientifiquement, de par son expérience médicale quotidienne de la situation du colonisé ; et d'autre part cette situation, il l'a historiquement vécue, il la vit encore, elle est pour lui une expérience personnelle dont il peut juger également de l'intérieur. Ayant décidé de s'écarter à la fois de la « grande erreur blanche » et du « grand mirage noir » il amorce une voie nouvelle, révolutionnaire: pour poser la question du colonisé et pour la résoudre, il occupe une place privilégiée : la conscience qu'il en a, la clarté de sa vision, renforcent la dureté de son engagement.

F. Fanon va choisir d'exercer en Algérie, pays du colonialisme par excellence, pour vivre et lutter parmi des colonisés comme lui. Le thème est repris et amplifié dans Racisme et Culture, conférence prononcée en 1956 au $1^{\text {er }}$ Congrès des Ecrivains Noirs. Cette fois, l'analyse devient plus aiguë, la mise en cause radicale, l'engagement ouvert et précis. Son diagnostic du racisme qui «n'est pas une découverte accidentelle» mais «entre dans un ensemble caractérisé, celui de l'exploitation d'un groupe d'hommes par un autre » implique une seule solution: «La fin logique de cette volonté de lutte est la libération totale du territoire national « «... La lutte est d'emblée totale, absolue ». Cette lutte n'est pas verbale. Depuis qu'il est médecin psychiâtre à I'hôpital de Blida, et plus encore après le déclenchement de l'insurrection, F. Fanon milite concrètement dans l'organisation, révolutionnaire algérienne. Dans le même temps il accomplit un remarquable travail médical, novateur sur tous les plans, profondément, viscéralement proche de ses malades en qui il voit avant tout les victimes du système qu'il combat. Il accumule les notes cliniques et les analyses sur les phénomènes de l'aliénation colonialiste vue au travers des maladies mentales. Il explore les traditions locales et ses rapports à la colonisation. Ce matériel capital est intact, mais lui aussi dispersé, et nous espérons pouvoir le réunir en un volume à part. 
Son travail de militant F.L.N. le fait bientôt repérer par la police française. A la fin de 1956, avant de rejoindre Tunis, il consacre par sa lettre de démission, un engagement total beaucoup plus ancien. C'est avec la lettre à un français, inédite, le seul texte qui témoigne de cette période dont nous avons fait le chapitre Pour l'Algérie. De l'expérience ainsi accumulée [9] au cœur même du combat, devait nâ̂tre plus tard l'Au $V$ de la Révolution algérienne.

À Tunis, F, Fanon est appelé à participer aux Services de Presse du F.L.N. Il est dans l'équipe des animateurs d'El Moudjahid dont ce sont les premiers numéros. Sans répit il s'attache à dénoncer la totalité, l'unité sans faille du système colonialiste, la solidarité qui, bon gré mal gré, lie ceux qui sont de son côté, tandis que s'exécute le génocide d'un million d'Algériens. Son analyse sur les intellectuels de gauche et la guerre d'Algérie outre la gauche française. Il y dénonce l'hypocrisie de ceux qui ne voient dans le colonialisme et ses suites, guerre, torture, qu'une excroissance monstrueuse qu'il suffit de circonscrire et de réprouver, alors qu'il s'agit d'un ensemble parfaitement logique, parfaitement cohérent, qui rend irrémédiablement complices tous ceux qui vivent en son sein.

Fanon a dès lors le moyen d'amplifier l'un de ses premiers thèmes: la conjonction de la lutte de tous les colonisés. L'un des premiers à envisager de manière concrète - non pas comme une «vision prophétique » mais comme un objectif de combat immédiat - l'unité de l'Afrique, il lie constamment le sort de la Révolution algérienne à celui de l'ensemble du continent, faisant de celle-ci l'avant-garde de la Révolution africaine. El Moudjahid développe constamment cette ligne : La Révolution algérienne et la libération de l'Afrique, ce titre donné à la brochure d'articles et de documents du F.L.N. la plus diffusée à cette époque indique bien l'importance que les révolutionnaires algériens lui accordent alors.

Les articles d'El Moudjahid n'étaient jamais signés. L'anonymat y était total. Les articles publiés ici, sous le contrôle de Madame F. Fanon, sont seulement ceux dont nous avons la certitude irréfutable qu'ils ont été écrits par F. Fanon. Certes sa collaboration ne s'est pas limitée à ces textes précis. Mais comme dans toute équipe, et particulièrement dans cette révolution en plein jaillissement, c'était un perpétuel travail d'osmose, d'interaction, de stimulations réciproques. Au moment même où la pensée de $F$. Fanon atteignait de nouvelles dimen- 
sions au contact du noyau créateur de la Révolution algérienne, elle transmettait à celle-ci de nouvelles impulsions. Nous avons groupé les textes ainsi reproduits sous le titre Libération de l'Afrique.

[10]

L'idée que Fanon formait de l'Afrique en marche se concrétise dans la mission qu'il mena dans les pays d'Afrique occidentale, après avoir été ambassadeur à Accra. Il devait notamment étudier les conditions d'une alliance plus étroite entre Africains, la levée de volontaires noirs, l'ouverture d'un nouveau front au Sud du Sahara... Les pages que nous publions dans ce dernier chapitre - Unité Africaine - sont celles d'un carnet de route inédit où ce plan prend toute sa clarté et sa violence.

F. Fanon rentra épuisé de cette mission : il était atteint de leucémie. Il consacra ses derniers efforts à rédiger Les damnés de la terre. Il devait mourir un an après avoir assisté à la chute de Lumumba dont il était l'ami et qui fut le leader africain dont la vision africaine était la plus proche de la sienne. Il gardait la certitude de la prochaine libération totale de l'Afrique, convaincu, comme il l'avait écrit dans l'An V de la Révolution algérienne que la révolution africaine avait créé « une situation irréversible». 
Pour la révolution africaine Écrits politiques

Première partie Le colonisé en question 


\author{
Pour la révolution africaine \\ Écrits politiques
}

Première partie. Le colonisé en question.

\title{
Chapitre 1
}

\section{Le « syndrome nord africain »1}

\section{Retour à la table des matières}

On dit volontiers que l'homme est sans cesse en question pour luimême, et qu'il se renie lorsqu'il prétend ne plus l'être. Or il semble qu'il doit être possible de décrire une dimension première de tous les problèmes humains. Plus précisément : que tous les problèmes que se pose l'homme au sujet de l'homme peuvent se ramener à cette question:

«N'ai-je pas, du fait de mes actes ou des mes abstentions, contribué à une dévalorisation de la réalité humaine? »

1 Texte publié dans la revue Esprit de février 1952. 
Question qui pourrait se formuler encore:

«Ai-je en toute circonstance réclamé, exigé l'homme qui est en moi?»

Je veux montrer dans ces lignes que, dans le cas particulier du Nord-Africain émigré en France, une théorie de l'inhumanité risque de trouver ses lois et ses corollaires.

Tous ces hommes qui ont faim, tous ces hommes qui ont froid, tous ces hommes qui ont peur...

Tous ces hommes qui nous font peur, qui écrasent l'émeraude jalouse de nos rêves, qui bousculent la fragile courbe de nos sourires, tous ces hommes en face de nous, qui ne [14] nous posent point de questions, mais à qui nous en posons d'étranges.

Quels sont-ils?

Je vous le demande, je me le demande. Quelles sont-elles, ces créatures affamées d'humanité qui s'arc-boutent aux frontières impalpables (mais je les sais d'expérience terriblement nettes) de la reconnaissance intégrale?

Quelles sont-elles, en vérité, ces créatures, qui se dissimulent, qui sont dissimulées par la vérité sociale sous les attributs de bicot, bounioule, arabe, raton, sidi, mon z'ami?

THĖSE I. - Que le comportement du Nord-Africain provoque souvent de la part du personnel médical une attitude de défiance quant à la réalité de sa maladie.

À l'exception des cas d'urgence: occlusion intestinale, blessures, accidents, le Nord-Africain se présente baigné de vague.

Il a mal dans le ventre, dans la tête, dans le dos, il a mal partout. Il souffre atrocement, son visage est éloquent, c'est une souffrance qui en impose. 
- Qu'est-ce que c'est, mon ami?

- Je vais mourir, monsieur le docteur.

La voix est cassée imperceptible.

- Où as-tu mal ?

- Partout, monsieur le docteur.

Surtout, n'exigez aucune précision: vous n'en obtiendriez pas. Par exemple, dans les douleurs d'allure ulcéreuse, il est important de connaître l'horaire des algies. Cette conformité aux catégories du temps, le Nord-Africain semble y être hostile. Ce n'est pas incompréhension, car souvent il vient accompagné d'un interprète. On dirait qu'il lui coûte de retourner là où il n'est plus. Le passé, pour lui, est un passé cuisant. Ce qu'il espère, c'est de ne jamais plus souffrir, de ne jamais plus être face à face avec ce passé. Cette douleur présente, qui mobilise ainsi les muscles de son visage, lui suffit. Il ne comprend pas qu'on veuille lui imposer, même par le souvenir, celle qui n'est déjà plus. Il ne comprend pas pourquoi le médecin lui pose tant de questions.

- Où as-tu mal ?

- Dans le ventre. (II montre alors thorax et abdomen.)

- À quel moment?

- Tout le temps.

[15]

- Même la nuit?

- Surtout la nuit.

- Tu as davantage mal la nuit que le jour, hein?

- Non, tout le temps.

- Mais plus la nuit que le jour?

- Non, tout le temps.

- Et où ça fait le plus mal ?

- Là. (Il montre alors thorax et abdomen.) 
Voilà, dehors des malades attendent et, chose grave, on a l'impression que le temps ne changerait rien à l'affaire. On part donc avec un diagnostic de probabilité et on institue corrélativement une thérapeutique approximative.

- Suis ce traitement pendant un mois. Si tu n'es pas amélioré, reviens me voir.

Alors deux solutions

$1^{\circ}$ Le consultant n'est pas immédiatement soulagé, et il retourne à la consultation trois ou quatre jours après. Cette démarche nous dresse contre lui, car nous savons qu'il y a un délai de prise en charge (nous nous excusons de cette expression) de la lésion par le médicament prescrit.

On le lui fait comprendre: plus exactement, on le lui dit. Mais notre malade ne nous a pas entendu. Il est sa douleur et il refuse de comprendre tout langage et le chemin n'est pas loin, qui conduit à cette proposition:

C'est parce que je suis Arabe qu'ils ne me soignent pas comme les autres.

$2^{\circ}$ Le consultant n'est pas immédiatement soulagé, mais il ne retourne pas dans le même service, ni chez le même médecin.

Il va ailleurs. Il part de ce principe qu'il faut, avant d'obtenir satisfaction, frapper à toutes les portes, et il frappe. Il frappe avec acharnement. Avec douceur. Avec naïveté. Avec rage.

Il frappe. On lui ouvre. Toujours on lui ouvre. Et il raconte sa douleur. Qui devient de plus en plus sienne. Il l'expose avec volubilité maintenant. Il la saisit dans l'espace, la met sous le nez du médecin. Il la prend, la touche de ses dix doigts, la développe, l'expose. Elle grossit à vue d'œil. Il la ramasse sur toute la surface de son corps et après quinze [16] minutes d'explications gestuelles, l'interprète (déroutant comme il se doit) nous traduit : il dit qu'il a mal au ventre. 
Toutes ces incursions dans l'espace, tous ces spasmes de la face, tous ces écarquillements ne voulaient exprimer qu'une douleur vague. Nous ressentons une sorte de frustration dans le domaine de l'explication. La comédie, ou le drame, recommence : diagnostic et thérapeutique approximatifs.

Il n'y a pas de raison pour que la roue s'arrête. Un jour, on lui fera une radiographie qui montrera un ulcère ou une gastrite. Ou qui ne montrera le plus souvent rien du tout. On dira de son algie qu'elle est «onctionnelle».

Cette notion est d'importance et mérite qu'on s'y arrête. Une chose est dite vague quand elle manque de consistance, de réalité objective. La douleur du Nord-Africain, à laquelle nous ne trouvons pas de base lésionnelle, est jugée inconsistante, irréelle. Or, le Nord-Africain est celui-qui-n'aime-pas-le-travail. De sorte que toutes ses démarches seront interprétées à partir de cet a priori.

Un Nord-Africain entre dans le service pour lassitude, asthénie, faiblesse. On lui institue un traitement actif à base de reconstituants. Au bout de vingt jours on décide sa sortie. Il se découvre alors une autre maladie.

- C'est le cour qui voltige là-dedans.

- C'est la tête qui éclate.

Devant cette crainte de la sortie, on arrive à se demander si la faiblesse pour laquelle il fut traité ne répondait pas à quelque vertige. On arrive à se demander si l'on ne fut pas le jouet de ce malade qu'on n'a jamais très bien compris. La suspicion fait son apparition. Dorénavant, on se méfiera des symptômes allégués.

La chose est nette en hiver: aussi certains services sont-ils littéralement submergés de Nord-Africains au moment des grands froids. Il fait si bon dans une salle d'hôpital.

Dans un service, un médecin tançait un Européen atteint de sciatique et qui circulait toute la journée dans les salles. Il lui expliquait que le repos représentait dans ce cas particulier la moitié de la thérapeutique. Avec les Nord-Africains ajouta-t-il à notre intention, le problè- 
me est différent : pas besoin de leur conseiller le repos, ils sont toujours au lit.

Devant cette douleur sans lésion, cette maladie répartie dans et sur tout le corps, cette souffrance continue, l'attitude [17] la plus facile et à laquelle on est plus ou moins rapidement conduit, est la négation de toute morbidité. À l'extrême, le Nord-Africain est un simulateur, un menteur, un tire-au-flanc, un fainéant, un feignant, un voleur ${ }^{2}$.

THĖSE II. - Que l'attitude du personnel médical est très souvent a prioriste. Le Nord-Africain n'arrive pas avec un fond commun à sa race, mais sur un fond bâti par l'Européen. Autrement dit, le NordAfricain, spontanément, du fait de son apparition, entre dans un cadre préexistant.

Depuis quelques années se manifeste une orientation médicale qu'on pourrait, très rapidement, dénommer néo-hypocratisme. Cette tendance veut qu'en face du malade, les médecins se soucient moins de porter un diagnostic d'organe qu'un diagnostic de fonction. Mais ce courant d'idées ne s'est pas encore imposé dans les chaires où la pathologie est enseignée. Il y a un vice de construction dans la pensée du praticien. Un vice extrêmement dangereux.

Nous allons le saisir sur le fait.

Je suis appelé en consultation d'urgence auprès d'un malade. Il est deux heures du matin. La chambre est sale, le malade est sale. Ses parents sont sales. Tout le monde pleure. Tout le monde crie. L'impression étrange que la mort n'est pas loin. Le jeune médecin expulse toute déroute de son âme. Il se penche «objectivement » sur ce ventre d'allure si chirurgicale.

Il touche, il palpe, il percute, il interroge, mais il n'obtient que des gémissements, il repalpe, derechef percute, et le ventre se contracte, se défend... Il ne « voit rien». Pourtant, si c'était chirurgical ? S'il laissait passer quelque chose? Son examen est négatif mais il n'ose repartir. Après pas mal d'hésitations, il dirigera son malade sur un cen-

2 Cf. La Sécurité sociale? C'est nous qui payons! 
tre avec le diagnostic de ventre chirurgical. Trois jours après, il voit arriver à son cabinet le « ventre chirurgical » souriant, complètement guéri. Et ce que le malade ignore, c'est qu'il y a une pensée médicale exigeante et que cette pensée, il l'a bafouée.

La pensée médicale va du symptôme à la lésion. Dans les illustres assemblées, dans les congrès internationaux de médecine, [18] on s'est mis d'accord sur l'importance des systèmes neurovégétatifs, du diencéphale, des glandes endocrines, des liaisons psychosomatiques, des sympathalgies, mais on continue à enseigner aux médecins que tout symptôme réclame sa lésion. Le malade est celui qui alléguant des céphalées, des bourdonnements d'oreille, des vertiges, présentera en même temps une hypertension artérielle. Mais, qu'à l'occasion de ces mêmes symptômes on ne trouve ni hypertension, ni tumeur intracrânienne, en tout cas rien de positif, le médecin alors verra la pensée médicale en défaut; et comme toute pensée est pensée de quelque chose, il verra le malade en défaut, - un malade indocile, indiscipliné, qui ignore la règle du jeu. Cette règle, pourtant si rigoureuse, qui s'énonce ainsi : Tout symptôme suppose une lésion.

Ce malade, que vais-je en faire? Du service où je l'avais envoyé pour intervention probable, il me revient avec un diagnostic de «syndrome nord-africain ». Et il est vrai que le jeune hypo de première arrivée entrera en contact avec Molière à travers les Nord-Africains de son service. Malade imaginaire! Si Molière (je vais dire une idiotie, mais toutes ces lignes ne font qu'expliciter, ne font que rendre flagrante une plus vaste idiotie), si Molière avait eu le privilège de vivre au $X X^{e}$ siècle, il n'eût certainement pas écrit Le Malade imaginaire, car il ne fait de doute pour personne qu'Argan est malade. Malade, il l'est activement :

Comment, coquine! Si je suis malade! Si je suis malade, impudente!

Syndrome nord-africain. Aujourd'hui, le Nord-Africain qui se présente à une consultation supporte le poids mort de tous ses compatriotes. Tous ceux qui n'avaient que des symptômes, tous ceux à propos de qui l'on disait : «Rien à se mettre sous la dent. » (Entendez: pas de lésion.) Mais le malade qui est là, en face de moi, ce corps que je suis forcé de supposer balayé par une conscience, ce corps qui n'est plus 
tout à fait corps ou du moins qui est doublement corps puisque ahuri d'épouvante, - ce corps qui me demande de l'écouter sans toutefois m'y attarder, - provoquera en moi une révolte.

- Où as-tu mal ?

- L'estomac. (Et il montre le foie.)

Je me fâche. Je lui dis que l'estomac est à gauche, que ce [19] qu'il montre c'est le siège du foie. Il ne se démonte point, il parcourt de la paume de la main ce ventre mystérieux:

- Tout ça fait mal.

Moi je sais que dans «tout ça», il y a trois organes; en toute rigueur cinq ou six. Que chaque organe a sa pathologie. Cette pathologie inventée par l'Arabe ne nous intéresse pas. C'est une pseudopathologie. L'Arabe est un pseudo-malade.

Tout Arabe est un malade imaginaire. Le jeune médecin ou le jeune étudiant qui n'a jamais vu un Arabe malade, sait (cf. la vieille tradition médicale) que « ces types sont des farceurs». Il y a quelque chose qui pourrait donner lieu à réflexion. En face d'un Arabe, l'étudiant ou le médecin est enclin à employer la deuxième personne du singulier. C'est gentil, nous dira-t-on... pour les mettre à l'aise... ils ont l'habitude... Je m'excuse, je me sens incapable d'analyser ce phénomène sans abandonner l'attitude objective que je me suis imposée.

C'est plus fort que moi, me disait un interne, je ne puis les aborder de la même façon que les autres malades.

Eh oui! c'est plus fort que moi. Si vous saviez ce qui dans ma vie est plus fort que moi. Si vous saviez ce qui dans ma vie me harcèle aux heures où les autres engourdissent leur cerveau. Si vous saviez... mais vous ne saurez pas.

Le personnel médical découvre l'existence d'un syndrome nordafricain. Non pas expérimentalement, mais selon une tradition orale. Le Nord-Africain prend place dans ce syndrome asymptomatique et se situe automatiquement sur un plan d'indiscipline ( $c f$. discipline médicale), d'inconséquence (par rapport à la loi : tout symptôme suppose une lésion), d'insincérité (il dit souffrir alors que nous savons ne pas exis- 
ter de raisons de souffrir). Il y a une idée mobile qui est là, à la limite de ma mauvaise foi, et quand l'Arabe se dévoilera à travers son langage:

«Monsieur le docteur, je vais mourir.»

Cette idée, après avoir parcouru quelques sinuosités, s'imposera, m'en imposera.

Décidément, ces types ne sont pas sérieux.

THĖSE III. - Que les meilleures volontés, les plus pures intentions demandent à être éclairées. - De la nécessité de porter un diagnostic de situation.

Le docteur Stern, dans un article sur la médecine psychosomatique, [20] reprenant les travaux de Heinrich Meng, écrit : « Il ne faut pas seulement trouver quel est l'organe atteint, de quelle nature sont les lésions organiques, si elles existent et quel microbe a envahi l'organisme; il ne suffit pas de connaître la « constitution somatique » du malade, mais il faut essayer de connaître ce que Meng appelle sa «situation», c'est-à-dire ses relations avec son entourage, ses occupations et ses préoccupations, sa sexualité, sa tension intérieure, son sentiment de sécurité ou d'insécurité, les dangers qui le menacent; et ajoutons aussi son évolution, l'histoire de sa vie. Il faut faire un « diagnostic de situation ${ }^{3}$.

Le docteur Stern nous propose un plan magnifique, nous le suivrons.

$1^{\circ}$ Relations avec l'entourage. - Faut-il vraiment en parler. N'y a-til pas un certain comique à parler des relations du Nord-Africain avec son entourage, en France? A-t-il des relations? A-t-il un entourage? N'est-il pas seul? Ne sont-ils pas seuls? Ne nous paraissent-ils pas absurdes, c'est-à-dire sans fondement, dans les trains ou dans les trolleybus? D'où viennent-ils? Où vont-ils? De temps à autre on les aperçoit travaillant à quelque édifice, mais on ne les voit pas, on les

3 Dr E. Stern. Médecine psycho-somatique, Psyché, janv.-févr. 1949, p. 128. 
aperçoit, on les entrevoit. Entourage? Relations? Il n'y a pas de contacts. Il n'y a que des heurts. Sait-on que ce mot contact renferme de doux et de poli (pas de policé, de poli) ? Y a-t-il contacts ? Y a-t-il relations?

$2^{\circ}$ Occupations et préoccupations. - Il travaille, il est occupé, il s'occupe, on l'occupe. Ses préoccupations? Je crois que le mot n'existe pas dans sa langue. Se préoccuper de quoi? En France on dit : il se préoccupe de trouver du travail ; en Afrique du Nord : il s'occupe à trouver du travail.

- Pardon madame, selon vous, quelles sont les préoccupations du Nord-Africain?

$3^{\circ}$ Sexualité. - Je vous entends, elle est faite de viol. Pour montrer à quel point une étude scotomisante peut être préjudiciable au dévoilement authentique d'un phénomène, je voudrais [21] reproduire quelques lignes d'une thèse de doctorat en médecine soutenue à Lyon en 1951 par le docteur Léon Mugniery:

«Dans la région stéphanoise, huit sur dix ont épousé des prostituées. La plupart des autres vivent en collage accidentel et de courte durée, quelquefois maritalement. Souvent ils hébergent pendant quelques jours une ou plusieurs prostituées auxquelles ils conduisent leurs amis.

« Car la prostitution semble jouer un rôle important dans le milieu nord-africain ${ }^{4}$... Elle découle du fort appétit sexuel qui est l'apanage de ces méridionaux au sang chaud. »

Plus loin :

4 Souligné par moi (p. 20). 
"Sans doute peut-on faire de nombreuses objections et montrer par des exemples multiples que les essais entrepris pour loger convenablement les Nord-Africains sont autant d'échecs.

«Il s'agit d'hommes jeunes pour la plupart (25 à 35 ans), avec de gros besoins sexuels, que les liens d'un mariage mixte ne peuvent fixer que temporairement, pour lesquels l'homosexualité est un penchant désastreux...

«Il existe peu de solutions à ce problème : ou bien, malgré les risques ${ }^{5}$ que comporte un certain envahissement par la famille arabe, on doit favoriser le regroupement de cette famille en France et faire venir des jeunes filles et des femmes arabes ; ou bien il faut tolérer pour eux des maisons closes...

«Si l'on ne devait pas tenir compte de ces facteurs, on risquerait de s'exposer de plus en plus à des tentatives de viol dont les journaux nous citent des exemples constants. La morale publique a sans doute plus à craindre de l'existence de ces faits que de l'existence des maisons de tolérance. »

Et pour terminer, le docteur Mugniery dénonce l'erreur du gouvernement français en écrivant en lettres capitales dans sa thèse cette phrase: «L'OCTROI DE LA CITOYENNETÉ FRANÇAISE CONFÉRANT L'ÉGALITÉ DES DROITS, SEMBLE AVOIR ÉTÉ TROP PRÉCOCE ET BASÉ SUR DES RAISONS SENTIMENTALES ET POLITIQUES, PLUS QUE SUR LE FAIT DE L'ÉVOLUTION SOCIALE ET INTELLECTUELLE D'UNE RACE À CIVILISATION PARFOIS RAFFINÉE, MAIS À COMPORTEMENT [22] SOCIAL, FAMILIAL ET SANITAIRE ENCORE PRIMITIF. » (p. 45).

Faut-il ajouter quelque chose, faut-il reprendre les unes après les autres ces phrases absurdes, faut-il rappeler au docteur Mugniery que si les Nord-Africains en France se contentent de prostituées, c'est parce que, premièrement, ils y trouvent des prostituées, et qu'ensuite

5 Souligné par moi (p. 20). 
ils n'y trouvent pas de femmes arabes (qui pourraient envahir la nation).

$4^{\circ}$ Sa tension intérieure. - Aucune réalité ? Autant parler de la tension intérieure d'une pierre. Tension intérieure! Cette blague!

$5^{\circ}$ Son sentiment de sécurité ou d'insécurité. - Le premier terme est à éliminer. Le Nord-Africain est en perpétuelle insécurité. Insécurité plurisegmentaire.

Je me demande à certains moments s'il ne serait pas bon de révéler au Français moyen que c'est un malheur d'être Nord-Africain. Le NordAfricain n'est jamais sûr. Il a des droits, me direz-vous, mais il ne les connaît pas. Ah! Ah! Il n'a qu'à les connaître. La connaissance. Eh oui! nous retombons sur nos pieds. Droits, Devoirs, Citoyenneté, Egalité, que de belles choses! Le Nord-Africain au seuil de la Nation française - qui est, nous dit-on, la sienne - vit dans le domaine politique, sur le plan civique, un imbroglio que personne ne veut voir en face. Quel rapport avec le Nord-Africain en milieu hospitalier? Justement, il y a un rapport.

\section{$6^{\circ}$ Les dangers qui le menacent.}

Menacé dans son affectivité,

Menacé dans son activité sociale,

Menacé dans son appartenance à la cité, le Nord-Africain réunit toutes les conditions qui font un homme malade.

Sans famille, sans amour, sans relations humaines, sans communion avec la collectivité, la première rencontre avec lui-même se fera sur un mode névrotique, sur un mode pathologique, il se sentira vidé, sans vie, en corps à corps avec la mort, une mort en deçà de la mort, une mort dans la vie, et qu'y a-t-il de plus pathétique que cet homme aux muscles robustes qui nous dit de sa voix véritablement cassé : «Docteur, je vais mourir. » 
$7^{\circ}$ Son évolution et l'histoire de sa vie. - Il vaudrait mieux dire I'histoire de sa mort. Une mort quotidienne.

Une mort dans le tram,

une mort à la consultation,

une mort avec les prostituées,

une mort au chantier,

une mort au cinéma,

une mort multiple dans les journaux,

une mort dans la crainte de tous les honnêtes gens de sortir après minuit.

Une mort,

oui une MORT.

Tout cela est bien beau, nous dira-t-on, mais quelle solution proposez-vous?

Vous le reconnaissez, ils sont vagues, amorphes

« Il faut qu'on soit dans leur dos. »

« Il faut les pousser hors de l'hôpital. »

«Si on les écoutait on prolongerait indéfiniment leur convalescence. »

«Ils ne savent pas s'expliquer. »

Et ils sont menteurs

et puis ils sont voleurs (voleur comme un Arabe)

et puis, et puis, et puis

l'Arabe est un voleur

tous les Arabes sont des voleurs. 
C'est une race feignante

sale

dégueulasse

$Y$ a rien à en faire

rien à en tirer

bien sûr, c'est dur pour eux d'être ainsi

d'être comme ça

mais, enfin, admettez que la faute ne vient pas de nous.

- Justement, la faute vient de nous.

Justement, la faute est TA faute.

Comment, des hommes vont et viennent le long d'un couloir que tu as construit pour eux, où tu n'as ménagé aucun banc où ils puissent se reposer, où tu as cristallisé un tas [24] d'épouvantails qui leur giflent rageusement le visage, où ils se blessent la face, la poitrine, le cœur.

Où ils ne trouvent pas de place

où tu ne leur fais pas de place

où il n'y a absolument pas place pour eux

et tu oses me dire que cela ne t'intéresse pas!

Que ce n'est pas ta faute!

Comment, cet homme que tu chosifies en l'appelant systématiquement Mohammed, que tu reconstruis, ou plutôt que tu dissous, à partir d'une idée, une idée que tu sais dégueulasse (tu sais bien, tu lui enlèves quelque chose, ce quelque chose pour lequel il n'y a pas bien longtemps tu étais prêt à tout quitter, même la vie) eh bien! cet homme-ci, tu n'as pas l'impression de le vider de sa substance?

Ils n'ont qu'à rester chez eux!

Eh oui! Voici le drame: ils n'ont qu'à rester chez eux. Seulement on leur a dit qu'ils étaient Français. Ils l'ont appris à l'école. Dans la rue. 
Dans les casernes. (Où il y avait des chaussures à leur pied). Sur les champs de bataille. On leur a introduit la France partout où, dans leur corps et dans leur «âme», il y avait place pour quelque chose d'apparemment grand.

Maintenant, on leur répète sur tous les tons qu'ils sont chez «nous ». Que s'ils ne sont pas contents, ils n'ont qu'à retourner dans leur kasbah. Car là encore, il y a un problème.

Quelles que soient les vicissitudes rencontrées par lui en France, prétendent certains, le Nord-Africain sera plus heureux chez lui...

On s'est aperçu en Angleterre que des enfants magnifiquement nourris, ayant chacun deux nurses entièrement à leur service, mais vivant en dehors du milieu familial, présentaient une morbidité deux fois plus importante que ceux moins bien nourris mais vivant au milieu de leurs parents. Sans aller si loin, qu'on pense à tous ceux qui mènent une vie sans avenir dans leur pays et qui refusent de belles situations à l'étranger. A quoi sert la belle situation quand elle n'aboutit pas à un milieu familial ou parental, quand elle ne permet pas l'épanouissement du « milieu »?

La science psychanalytique tient l'expatriement pour un phénomène morbide. Ce en quoi elle a parfaitement raison.

[25]

Ces considérations nous permettent de conclure :

$1^{\circ}$ Le Nord-Africain ne sera jamais plus heureux en Europe que chez lui car il lui est demandé de vivre sans la matière même de son affectivité. Coupé de ses origines et coupé de ses fins, il est une chose jetée dans le grand fracas, courbée sous la loi de l'inertie.

$2^{\circ}$ Il y a dans ce propos une mauvaise foi manifeste et abjecte. Si le standard de vie (?) mis à la disposition du Nord-Africain en France est supérieur à celui auquel il était habitué chez lui, cela veut dire qu'il reste beaucoup à faire dans son pays, dans cette « autre partie de la France ».

Qu'il y a des immeubles à construire, des écoles à ouvrir, des routes à tracer, des taudis à démolir, des villes à faire surgir de terre, des hommes et des femmes, des enfants et des enfants à garnir de sourires. 
Cela veut dire qu'il y a du travail là-bas, du travail humain, c'est-àdire du travail qui soit la signification d'un foyer. Pas celle d'une chambre ou d'un casernement. Cela veut dire que sur tout le territoire de la nation française (métropole et Union française), il y a des pleurs à sécher, des attitudes inhumaines à combattre, des mon z'ami à rendre inadmissibles, des hommes à humaniser, des rues Moncey 6 à rendre à la circulation.

Votre solution, monsieur?

Ne me poussez pas à bout. Ne m'obligez pas à vous dire ce que vous devriez savoir, monsieur. Si TU ne réclames pas l'homme qui est en face de toi, comment veux-tu que je suppose que tu réclames l'homme qui est en toi?

Si TU ne veux pas l'homme qui est en face de toi, comment croiraije à l'homme qui est peut-être en toi ?

Si TU n'exiges pas l'homme, si TU ne sacrifies pas l'homme qui est en toi pour que l'homme qui est sur cette terre soit plus qu'un corps, plus qu'un Mohammed, par quel tour de passepasse faudra-t-il que j'acquière la certitude que, toi aussi, tu es digne de mon amour?

6 Rue d'un quartier pauvre de Lyon qu'habitent de nombreux Nord-Africains. 


\author{
Pour la révolution africaine \\ Écrits politiques
}

Première partie. Le colonisé en question.

\title{
Chapitre 2
}

\section{Antillais et Africains ?}

\section{Retour à la table des matières}

Il y a deux ans, je terminais un ouvrage 8 sur le problème de I'homme de couleur dans le monde blanc. Je savais qu'il ne fallait absolument pas amputer la réalité. Je n'ignorais point qu'au sein même du «peuple noir», cette entité, se pouvaient distinguer des mouvements malheureusement fort inesthétiques. Je veux dire, par exemple, que souvent l'ennemi du nègre n'est pas le blanc, mais son congénère. C'est pourquoi je signalais la possibilité d'une étude qui contribuerait à la dissolution des complexes affectifs susceptibles d'opposer Antillais et Africains.

7 Texte publié dans la Revue Esprit de février 1955.

8 Peau noire et masques blancs (coll. Esprit, éd. du Seuil). [Livre disponible dans Les Classiques des sciences sociales. JMT.] 
Avant d'entrer dans le débat nous voudrions faire remarquer que cette histoire de nègre est une sale histoire. Une histoire à vous soulever l'estomac. Une histoire en face de laquelle on se trouve totalement démuni si on accepte les prémisses des salauds. Et quand je dis que l'expression: «peuple noir » est une entité, j'indique par là que, les influences culturelles exclues, il ne reste plus rien. Il y a autant de différence entre un Antillais et un Dakarien qu'entre un Brésilien et un Madrilène. Ce qu'on cherche en englobant tous les nègres sous le terme «peuple noir » c'est à leur enlever toute possibilité d'expression individuelle. Ce qu'on cherche [28] ainsi, c'est à les mettre dans l'obligation de répondre à l'idée qu'on se fait d'eux. Que serait le « peuple blanc »? Ne voit-on donc pas qu'il ne peut y avoir qu'une race blanche? Faut-il donc que j'explique la différence qui existe entre nation, peuple, patrie, communauté ? Quand on dit «peuple noir», on suppose systématiquement que tous les noirs sont d'accord sur certaines choses: qu'il existe, entre eux, un principe de communion. La vérité, est qu'il n'y a rien, a priori, qui puisse laisser supposer l'existence d'un peuple noir. Qu'il y ait un peuple africain, je le crois ; qu'il y ait un peuple antillais, je le crois. Mais quand on me parle de «ce peuple noir » j'essaie de comprendre. Alors, malheureusement, je comprends qu'il y a là une source de conflits. Alors j'essaie de détruire cette source.

On me verra employer des termes comme: culpabilité métaphysique ou folie de pureté. Je demanderai au lecteur de ne point s'en étonner : ce sera exact dans la mesure où l'on comprendra que l'important ne pouvant être atteint ou, plus précisément, l'important n'étant pas souhaité, c'est sur le contingent que l'on se rabat. C'est une des lois de la récrimination et de la mauvaise foi. Retrouver l'important sous le contingent, telle est l'urgence.

De quoi est-il question ici? Je dis qu'en quinze ans il s'est produit une révolution dans les relations antillo-africaines. Je veux montrer en quoi consiste cet événement.

En Martinique, il est rare de constater des positions raciales tenaces. Le problème racial est recouvert par une discrimination économique et, dans une classe sociale déterminée, il est surtout producteur d'anecdotes. Les relations ne sont pas altérées par les accentuations épidermiques. En dépit de la plus ou moins grande charge de mélanine, il existe un accord tacite permettant aux uns et aux autres de se re- 
connaître comme médecins, commerçants, ouvriers. Un noir ouvrier sera du côté du mulâtre ouvrier contre le noir bourgeois. On a ici la preuve que les histoires raciales ne sont qu'une superstructure, qu'un manteau, qu'une sourde émanation idéologique devêtant une réalité économique. 9

[29]

Là-bas, quand on remarque que tel individu est tout de même très noir on le fait sans mépris, sans haine. Il faut être habitué à ce qu'on appelle l'esprit martiniquais pour saisir ce qui se passe. Jankelevitch a montré que l'ironie était une des formes de la bonne conscience. Il est exact que l'ironie aux Antilles est un mécanisme de défense contre la névrose. Un Antillais, principalement un intellectuel qui ne se trouve plus sur le plan de l'ironie, découvre sa négritude. Ainsi donc, alors qu'en Europe l'ironie protège de l'angoisse existentielle en Martinique elle protège de la prise de conscience de la négritude. La mission consiste à déplacer le problème, à mettre le contingent à sa place et à laisser au Martiniquais le choix des valeurs suprêmes. On voit tout ce qu'on pourrait dire en envisageant cette situation à partir des étapes kierkegaardiennes. On voit aussi qu'une étude de l'ironie aux Antilles est capitale pour la sociologie de cette région. Presque toujours l'agressivité là-bas est ouatée d'ironie 10.

Pour faciliter notre exposé, il nous semble intéressant de distinguer dans l'histoire antillaise deux périodes: avant et après la guerre de 1939-1945.

9 Disons que les concessions que nous avons faites sont fictives. Philosophiquement et politiquement il n'y a pas de peuple africain, mais un monde africain. De même un monde antillais. Par contre, on peut dire qu'il existe un peuple juif ; mais pas de race juive.

10 Voir par exemple le Carnaval et les chansons composées à cette occasion. 


\section{Avant la guerre}

Avant 1939, l'Antillais se disait heureux 11 , tout au moins croyait l'être. Il votait, allait à l'école quant il le pouvait, suivait les processions, aimait le rhum et dansait la biguine. Ceux qui avaient le privilège d'aller en France parlaient de Paris, de Paris, enfin de la France. Et ceux qui n'avaient pas le privilège de connaître Paris se laissaient bercer.

Il y avait aussi les fonctionnaires travaillant en Afrique. A travers eux on voyait un pays de sauvages, de barbares, d'indigènes, de boys. Il faut dire certaines choses si l'on ne veut pas fausser le problème. Le fonctionnaire métropolitain, revenant d'Afrique, nous a habitués à des clichés: sorciers, féticheurs, [30] tam-tam, bonhomie, fidélité, respect du blanc, arriération. Le drame c'est que le fonctionnaire antillais ne parle pas autrement de l'Afrique et, comme le fonctionnaire c'est non seulement l'administrateur des colonies, mais le gendarme, le douanier, le greffier, le militaire, à tous les échelons de la société antillaise se forme, se systématise, se durcit un irréductible sentiment de supériorité sur l'Africain. Chez tout Antillais, avant la guerre de 1939, il n'y avait pas seulement la certitude d'une supériorité sur l'Africain, mais celle d'une différence fondamentale. L'Africain était un nègre et l'Antillais un Européen.

Ces choses, tout le monde a l'air de ne les point ignorer. mais en vérité on n'en tient absolument pas compte.

Avant 1939, l'Antillais engagé volontaire dans la Coloniale, illettré ou sachant lire et écrire, servait dans une unité européenne, alors que l'Africain, à l'exception des originaires des cinq territoires, servait dans une unité indigène. Le résultat sur lequel nous voulons attirer l'attention c'est que quel que fût le domaine considéré, l'Antillais était supérieur à l'Africain, d'une autre essence, assimilé au métropolitain. Mais comme à l'extérieur il était un tout petit peu africain puisque, ma

11 On pourrait dire : comme la petite bourgeoise française à cette époque, mais ce n'est pas là notre perspective. Ce que nous voulons ici c'est étudier le changement d'attitude de l'Antillais vis-à-vis de la négritude. 
foi, noir, il était obligé - réaction normale dans l'économie psychologique - de durcir ses frontières afin d'être à l'abri de toute méprise.

Disons que, non content d'être supérieur à l'Africain, l'Antillais le méprisait, et si le blanc pouvait se permettre certaines libertés avec l'indigène, l'Antillais, lui, ne le pouvait absolument pas. C'est que, entre blancs et Africains, il n'y avait pas besoin de rappel à l'ordre, ça crevait les yeux. Mais quel drame si tout à coup l'Antillais était pris pour un Africain! ...

Disons aussi que cette position de l'Antillais était authentifiée par l'Europe. L'Antillais n'était pas un nègre, c'était un Antillais, c'est-àdire un quasi-métropolitain. Par cette attitude le blanc donnait raison à l'Antillais dans son mépris de l'Africain. En somme le nègre habitait l'Afrique.

En France, avant 1940, quand on présentait un Antillais dans une société bordelaise ou parisienne on ajoutait toujours : originaire de la Martinique. Je dis Martinique car, s'en est-on aperçu? la Guadeloupe, on ne saura jamais pourquoi, était considérée comme un pays de sauvages. Aujourd'hui encore il nous arrive, en 1952, d'entendre un Martini [31] quais nous affirmer qu'ils (les Guadeloupéens) sont plus sauvages que nous.

L'Africain, lui, était en Afrique le représentant réel de la race nègre. D'ailleurs, lorsqu'un patron réclamait un trop lourd effort à un Martiniquais il se voyait répondre : «Si vous voulez un nègre, allez le chercher en Afrique », entendant par là que les esclaves et les travailleurs de force se recrutaient ailleurs. Là-bas, chez les nègres.

L'Africain, lui, infériorisé, méprisé, à part quelques rares «évolués », croupissait dans le labyrinthe de son épiderme. Comme ont le voit, les positions étaient nettes: d'un côté le nègre, l'Africain; de l'autre l'Européen et l'Antillais. L'Antillais était un noir, mais le nègre était en Afrique.

En 1939, aucun Antillais aux Antilles ne se déclarait nègre, ne se réclamait nègre. Quand il le faisait, c'était toujours dans ses relations avec un blanc. C'est le blanc, le « mauvais blanc » qui l'obligeait à revendiquer sa couleur, plus véritablement à la défendre. Mais on peut affirmer qu'aux Antilles en 1939, aucune revendication spontanée de la négritude ne jaillissait. 
C'est alors que successivement vont se produire trois événements.

Et d'abord l'arrivée de Césaire.

Pour la première fois, on verra un professeur de lycée, donc apparemment un homme digne, simplement dire à la société antillaise « qu'il est beau et bon d'être nègre ». Pour sûr, c'était un scandale. On a raconté à cette époque qu'il était un peu fou et ses camarades de promotion se faisaient fort de donner des détails sur sa prétendue maladie.

Quoi de plus grotesque, en effet, qu'un homme instruit, un diplômé, ayant donc compris pas mal de choses, entre autres que "c'était un malheur d'être nègre», clamant que sa peau est belle et que le " grand trou noir » est source de vérité? Ni les mulâtres, ni les nègres ne comprirent ce délire. Les mulâtres parce qu'ils s'étaient échappés de la nuit, les nègres parce qu'ils aspiraient à en sortir. Deux siècles de vérité blanche donnaient tort à cet homme. Il fallait qu'il fût fou car il ne pouvait être question qu'il eût raison.

L'émoi apaisé, tout sembla reprendre son allure première... Et Césaire allait avoir tort quand le deuxième événement se produisit : je veux parler de la défaite française.

La France vaincue, I'Antillais, en un sens, assistait au [32] meurtre du père. Cette défaite nationale aurait pu être vécue comme elle le fut dans la métropole, mais une bonne partie de la flotte française resta bloquée aux Antilles pendant les quatre années de l'occupation allemande. Ici, je voudrais attirer l'attention du lecteur. Je crois qu'il est nécessaire de saisir l'importance historique de ces quatre années.

Avant 1939, il y avait en Martinique environ deux mille Européens. Ces Européens avaient des fonctions définies, étaient intégrés à la vie sociale, intéressés à l'économie du pays. Or, du jour au lendemain, la seule ville de Fort-de-France fut submergée par près de dix mille Européens à mentalité raciste certaine mais jusqu'alors latente. Je veux dire que les marins du Béarn ou de l'Emile-Bertin, auparavant à Fortde-France pendant huit jours n'avaient pas le temps de manifester leurs préjugés raciaux. Les quatre années pendant lesquelles ils furent obligés de vivre fermés sur eux-mêmes, inactifs, en proie à l'angoisse quand ils songaient à leurs parents laissés en France, victimes souvent du désespoir quand à l'avenir, leur permirent de jeter bas un masque, tout compte fait assez superficiel et de se comporter en « authentiques racistes ». 
Ajoutons que l'économie antillaise subit un rude coup car il fallut trouver, là encore sans transition, alors qu'aucune importation n'est possible, de quoi nourrir dix mille hommes. De plus, beaucoup de ces marins et militaires purent faire venir leur femme et leurs enfants qu'il fallut loger. La Martinique eut sa crise de logement après sa crise économique. Le Martiniquais tint pour responsables de tout cela ces blancs racistes. L'Antillais, devant ces hommes qui le méprisaient, se mit à douter de ses valeurs. L'Antillais faisait sa première expérience métaphysique.

Et puis ce fut la France libre. De Gaulle, à Londres, parlait de trahison, de militaires qui rendaient leur épée avant même de l'avoir tirée. Tout cela contribua à persuader les Antillais que la France, la leur, n'avait pas perdu la guerre mais que des traîtres l'avaient vendue. Et ces traîtres où étaient-ils, sinon camouflés aux Antilles? Et l'on vit cette chose extraordinaire: des Antillais refusant de se découvrir pendant l'exécution de la Marseillaise. Quel Antillais ne se rappelle ces jeudis soirs où, sur l'esplanade de la Savane, des patrouilles de marins armés réclamaient le silence et le [33] garde-à-vous quand on jouait l'hymne national ? Que s'était-il donc passé ?

Par un processus qui est facile à comprendre, les Antillais avaient assimilé la France des marins à la mauvaise France et la Marseillaise que respectaient ces hommes n'était pas la leur. Il ne faut pas oublier que ces militaires étaient racistes. Or « il ne fait de doute pour personne que le véritable Français n'est pas raciste, c'est-à-dire ne considère pas l'Antillais comme un nègre ». Puisqu'eux le faisaient c'est qu'ils n'étaient pas de véritables Français. Qui sait, peut-être des Allemands? Et de fait, systématiquement, le marin fut considéré comme un Allemand. Mais la conséquence qui nous intéresse est la suivante: devant dix mille racistes, l'Antillais se trouva obligé de se défendre. Sans Césaire cela lui aurait été difficile. Or, Césaire était là et l'on entonna avec lui ce chant, autrefois odieux, qu'il est beau et bon et bien d'être nègre !...

Pendant deux ans l'Antillais défendit pied à pied sa « couleur vertueuse » et sans s'en douter dansait au-dessus du précipice. Car enfin, si la couleur noire est vertueuse, je serai d'autant plus vertueux que je serai noir! Alors sortirent de l'ombre les très noirs, les «bleus», les purs. Et Césaire, chantre fidèle, répétait : « qu'on a beau peindre blanc le tronc de l'arbre, les racines en-dessous demeurent noires ». Alors il 
devint réel que non seulement le noir-couleur était valorisé, mais le noir fiction, le noir-idéal, le noir dans l'absolu, le noir-primitif, le nègre. Qu'était-ce, sinon provoquer chez l'Antillais une refonte totale de son monde, une métamorphose de son corps? Qu'était-ce, sinon exiger de lui une activité axiologique inversée, une valorisation du rejeté ?

Mais I'histoire continuait. En 1943, lassés par un ostracisme auquel ils n'étaient pas habitués, irrités, affamés, les Antillais, autrefois répartis en groupes sociologiques fermés brisaient toutes barrières, se mettaient d'accord sur certaines choses, entre autres que ces Allemands avaient dépassé les limites et arrachaient, appuyés par l'armée locale, le ralliement à la France libre. L'amiral Robert, « cet autre Allemand », cédait. C'est ici que se situe le troisième événement.

On peut dire que les manifestations de la Libération, qui eurent lieu aux Antilles, en tout cas en Martinique, dans les mois de juillet et d'août 1943, furent la conséquence de la naissance du prolétariat. La Martinique systématisait pour la [34] première fois sa conscience politique. Il est logique que les élections qui suivirent la Libération aient délégué deux députés communistes sur trois. En Martinique, la première expérience métaphysique, ou si l'on préfère ontologique, coïncida avec la première expérience politique. Comte faisait du prolétaire un philosophe systématique, le prolétaire martiniquais, lui, est un nègre systématisé.

\section{Après la guerre}

Ainsi donc l'Antillais, après 1945, a changé ses valeurs. Alors qu'avant 1939 il avait les yeux fixés sur l'Europe blanche, alors que pour lui le bien était l'évasion hors de sa couleur, il se découvre en 1945, non seulement un noir, mais un nègre et c'est vers la lointaine Afrique qu'il lancera désormais ses pseudopodes. L'Antillais en France rappelait à tout instant qu'il n'était pas un nègre: à partir de 1945, l'Antillais, en France, rappellera à tout instant qu'il est un nègre.

Pendant ce temps l'Africain continuait sa route. Il n'était pas déchiré, il n'avait pas à se situer simultanément vis-à-vis de l'Antillais et vis-à-vis de l'Européen. Ces derniers étaient à mettre dans le même sac, le sac des affameurs, des exploiteurs, des salauds. Il y avait eu 
bien sûr Eboué, Antillais cependant, qui, à la conférence de Brazzaville, avait parlé aux Africains en leur disant: « Mes chers frères. » Et cette fraternité n'était pas évangélique, elle était basée sur la couleur. Les Africains avaient adopté Eboué. Il était leur. Les autres Antillais pouvaient venir, leurs prétentions, de toubabs étaient connues. Or, à leur grand étonnement, les Antillais arrivant en Afrique après 1945, se présentèrent les mains suppliantes, le dos courbé, effondrés. Ils arrivaient en Afrique le cœur chargé d'espoir, désireux de retrouver la source, de se nourrir aux authentiques mamelles de la terre africaine. Les Antillais, fonctionnaires et militaires, avocats et médecins, débarquant à Dakar, étaient malheureux de n'être pas assez noirs. Il y a quinze ans, ils disaient aux Européens : «Ne faites pas attention à ma peau noire, c'est le soleil qui m'a brûlé, mon âme est blanche comme la vôtre. » A partir de 1945, ils changent de propos. Ils disent aux Africains : «Ne faites pas attention à ma peau blanche, mon âme est noire comme la vôtre et c'est ce qui importe.»

[35]

Mais les Africains leur en voulaient trop pour que le bouleversement fût aussi facile. Reconnus dans leur noirceur, dans leur obscurité, dans ce qui, il y a quinze ans, était la faute, ils dénièrent à l'Antillais toute velléité dans ce domaine. Ils se découvraient enfin possesseurs de la vérité, porteurs séculaires, d'une inaltérable pureté. Ils renvoyèrent l'Antillais de l'autre côté en lui rappelant qu'eux n'avaient pas déserté, qu'eux n'avaient pas trahi, qu'ils avaient peiné, souffert, lutté sur la terre africaine. L'Antillais avait dit non au blanc, l'Africain disait non à l'Antillais.

Ce dernier faisait sa deuxième expérience métaphysique. Il ressentit alors le désespoir. Hanté par l'impureté, accablé par la faute, sillonné par la culpabilité, il vécut le drame de n'être ni blanc ni nègre.

Il cria, composa des poèmes, chanta l'Afrique, l'Afrique terre dure et belle, l'Afrique explosion de colère, affairement tumultueux, éclaboussement, l'Afrique terre de vérité. A I'Institut des Langues Orientales à Paris il apprit le Bambara. L'Africain, dans sa majesté, condamnait toutes démarches. L'Africain prenait sa revanche et l'Antillais payait...

Si nous essayons maintenant d'expliquer et de résumer la situation, nous pouvons dire qu'en Martinique, avant 1939, il n'y avait pas d'un cô- 
té le nègre et de l'autre côté le blanc, mais des gammes colorées dont il était facile de franchir rapidement les intervalles. Il suffisait d'avoir des enfants avec un moins noir que soi. Il n'y avait pas de barrière raciale, pas de discriminations. Il y avait ce piment ironique, si caractéristique de la mentalité martiniquaise.

Mais en Afrique, la discrimination était réelle. Là-bas le nègre, l'Africain, l'indigène, le noir, le sale était rejeté, méprisé, maudit. Làbas il y avait amputation, il y avait méconnaissance d'humanité.

Jusqu'en 1939 l'Antillais vivait, pensait, rêvait (nous l'avon montré, dans notre essai Peau noire, masques blancs) composait des poèmes, écrivait des romans exactement comme l'aurait fait un blanc. On comprend maintenant pourquoi il ne lui était pas possible de chanter comme les poètes africains la nuit noire, «La femme noire aux talons roses». Avant Césaire la littérature antillaise est une littérature d'Européens. L'Antillais s'identifiait au blanc, adoptait une attitude de blanc, « était un blanc».

Après que l'Antillais fut obligé, sous la pression d'Européens [36] racistes, d'abandonner des positions somme toute fragiles, puisque absurdes, puisque inexactes, puisque aliénantes, va naître une nouvelle génération. L'Antillais 1945 est un nègre...

Il y a dans Cahier d'un retour au pays natal une période africaine car :

À force de penser au Congo

Je suis devenu un Congo bruissant de forêts et de fleuve 12

Alors, tourné vers l'Afrique, l'Antillais va la héler. Il se découvre fils d'esclaves transplanté, il sent la vibration de l'Afrique au plus profond de son corps et n'aspire qu'à une chose: qu'à plonger dans le grand « trou noir ».

Il semble donc que l'Antillais, après la grande erreur blanche, soit en train de vivre maintenant dans le grand mirage noir.

12 Cahier d'un retour au pays natal, page 49. 
[37]

\author{
Pour la révolution africaine \\ Écrits politiques
}

Deuxième partie

Racisme et culture 13

Retour à la table des matières

13 Texte de l'intervention de Frantz Fanon au $1^{\text {er }}$ Congrès des Ecrivains et Artistes Noirs à Paris, septembre 1956. Publié dans le N Spécial de Présence Africaine, juin-novembre 1956. 
La réflexion sur la valeur normative de certaines cultures décrétée unilatéralement mérite de retenir l'attention. L'un des paradoxes rapidement rencontré est le choc en retour de définitions égocentristes, sociocentristes.

Est affirmée d'abord l'existence de groupes humains sans culture: puis de cultures hiérarchisées ; enfin la notion de relativité culturelle.

De la négation globale à la reconnaissance singulière et spécifique. C'est précisément cette histoire morcelée et sanglante qu'il nous faut esquisser au niveau de l'anthropologie culturelle.

Il existe, pouvons-nous dire, certaines constellations d'institutions, vécues par des hommes déterminés, dans le cadre d'aires géographiques précises qui à un moment donné ont subi l'assaut direct et brutal de schèmes culturels différents. Le développement technique, généralement élevé, du groupe social ainsi apparu l'autorise à installer une domination organisée. L'entreprise de déculturation se trouve être le négatif d'un plus gigantesque travail d'asservissement économique voire biologique.

La doctrine de la hiérarchie culturelle n'est donc qu'une modalité de la hiérarchisation systématisée poursuivie de façon implacable.

La théorie moderne de l'absence d'intégration corticale des peuples coloniaux en est le versant anatomo-physiologique. L'apparition du racisme n'est pas fondamentalement déterminante. Le racisme n'est pas un tout mais l'élément le plus visible, le plus quotidien, pour tout dire, à certains moments, le plus grossier d'une structure donnée.

Étudier les rapports du racisme et de la culture c'est se poser la question de leur action réciproque. Si la culture est l'ensemble des comportements moteurs et mentaux né de la rencontre de l'homme 
avec la nature et avec son semblable on doit dire que le racisme est bel et bien un élément culturel. [40] Il y a donc des cultures avec racisme et des cultures sans racisme.

Cet élément culturel précis ne s'est cependant pas enkysté. Le racisme n'a pas pu se scléroser. Il lui a fallu se renouveler, se nuancer, changer de physionomie. Il lui a fallu subir le sort de l'ensemble culturel qui l'informait.

Le racisme vulgaire, primitif, simpliste prétendait trouver dans le biologique, les Ecritures s'étant révélées insuffisantes la base matérielle de la doctrine. Il serait fastidieux de rappeler les efforts entrepris alors: forme comparée du crâne, quantité et configuration des sillons de l'encéphale, caractéristiques des couches cellulaires de l'écorce, dimensions des vertèbres, aspect microscopique de l'épiderme, etc...

Le primitivisme intellectuel et émotionnel apparaissait comme une conséquence banale, une reconnaissance d'existence.

De telles affirmations, brutales et massives, cèdent la place à une argumentation plus fine. Ça et là toutefois se font jour quelques résurgences. C'est ainsi que la «labilité émotionnelle du Noir», «l'intégration sous-corticale de l'Arabe « «a culpabilité quasi générique du Juif » sont des données que l'on retrouve chez quelques écrivains contemporains. La monographie de J. Carothers, par exemple, patronnée par l'O.M.S. fait état à partir d'« arguments scientifiques » d'une lobotomie physiologique du Noir d'Afrique.

Ces positions séquellaires tendent en tous cas à disparaître. Ce racisme qui se veut rationnel, individuel, déterminé génotypique et phénotypique se transforme en racisme culturel. L'objet du racisme n'est plus l'homme particulier mais une certaine forme d'exister. A l'extrême on parle de message, de style culturel. Les "valeurs occidentales» rejoignent singulièrement le déjà célèbre appel à la lutte de la « croix contre le croissant $»$.

Certes l'équation morphologique n'a pas disparu totalement, mais les événements des trente dernières années ont ébranlé les convictions les plus encapsulées, bouleversé l'échiquier, restructuré un grand nombre de rapports. 
Le souvenir de nazisme, la commune misère d'hommes différents, le commun asservissement de groupes sociaux importants, l'apparition de « colonies européennes » c'est-à-dire l'institution d'un régime colonial en pleine terre d'Europe, la prise de conscience des travailleurs des pays colonisateurs et [41] racistes, l'évolution des techniques, tout cela a modifié profondément l'aspect du problème.

Il nous faut chercher, au niveau de la culture, les conséquences de ce racisme.

Le racisme, nous l'avons vu, n'est qu'un élément d'un plus vaste ensemble: celui de l'oppression systématisée d'un peuple. Comment se comporte un peuple qui opprime? Ici des constantes sont retrouvées.

On assiste à la destruction des valeurs culturelles, des modalités d'existence. Le langage, l'habillement, les techniques sont dévalorisées. Comment rendre compte de cette constante? Les psychologues qui ont tendance à tout expliquer par des mouvements de l'âme, prétendent retrouver ce comportement au niveau des contacts entre particuliers: critique d'un chapeau original, d'une façon de parler, de marcher...

De pareilles tentatives ignorent volontairement le caractère incomparable de la situation coloniale. En réalité les nations qui entreprennent une guerre coloniale ne se préoccupent pas de confronter des cultures. La guerre est une gigantesque affaire commerciale et toute perspective doit être ramenée à cette donnée. L'asservissement, au sens le plus rigoureux, de la population autochtone est la première nécessité.

Pour cela il faut briser ses systèmes de référence. L'expropriation, le dépouillement, la razzia, le meurtre objectif se doublent d'une mise à sac des schèmes culturels ou du moins conditionnent cette mise à sac. Le panorama social est destructuré, les valeurs bafouées, écrasées, vidées.

Les lignes de forces, écroulées, n'ordonnent plus. En face un nouvel ensemble, imposé, non pas proposé mais affirmé, pesant de tout son poids de canons et de sabres.

La mise en place du régime colonial n'entraîne pas pour autant la mort de la culture autochtone. Il ressort au contraire de l'observation historique que le but recherché est davantage une agonie continuée 
qu'une disparition totale de la culture pré-existante. Cette culture, autrefois vivante et ouverte sur l'avenir, se ferme, figée dans le statut colonial, prise dans le carcan de l'oppression. A la fois présente et momifiée elle atteste contre ses membres. Elle les définit en effet sans appel. La momification culturelle entraîne une momification de la pensée individuelle. L'apathie si universellement signalée des peuples coloniaux n'est que la conséquence logique de cette opération. Le reproche de l'inertie constamment adressé [42] à «l'indigène» est le comble de la mauvaise foi. Comme s'il était possible à un homme d'évoluer autrement que dans le cadre d'une culture qui le reconnaît et qu'il décide d'assumer.

C'est ainsi que l'on assiste à la mise en place d'organismes archaïques, inertes, fonctionnant sous la surveillance de l'oppresseur et calqués caricaturalement sur des institutions autrefois fécondes...

Ces organismes traduisent apparemment le respect de la tradition, des spécificités culturelles, de la personnalité du peuple asservi. Ce pseudo respect s'identifie en fait au mépris le plus conséquent, au sadisme le plus élaboré. La caractéristique d'une culture est d'être ouverte, parcourue de lignes de force spontanées, généreuses, fécondes. L'installation «d'hommes sûrs » chargés d'exécuter certains gestes est une mystification qui ne trompe personne. C'est ainsi que les djemaas Kabyles nommées par l'autorité française ne sont pas reconnues par les autochtones. Elles sont doublées d'une autre djemaa élue démocratiquement. Et naturellement la deuxième dicte la plupart du temps sa conduite à la première.

Le souci constamment affirmé de « respecter la culture des populations autochtones $\gg$ ne signifie donc pas la prise en considération des valeurs portées par la culture, incarnées par les hommes. Bien plutôt on devine dans cette démarche une volonté d'objectiver, d'encapsuler, d'emprisonner, d'enkyster. Des phrases telles que: « je les connais », «ils sont comme cela » traduisent cette objectivation maximum réussie. Ainsi je connais les gestes, les pensées qui définissent ces hommes.

L'exotisme est une des formes de cette simplification. Dès lors, aucune confrontation culturelle ne peut exister. Il y a d'une part une culture à qui l'on reconnaît des qualités de dynamisme, d'épanouissement, de profondeur. Une culture en mouvement, en per- 
pétuel renouvellement. En face on trouve des caractéristiques, des curiosités, des choses, jamais une structure.

Ainsi dans une première phase l'occupant installe sa domination, affirme massivement sa supériorité. Le groupe social, asservi militairement et économiquement est déshumanisé selon une méthode polydimensionnelle.

Exploitation, tortures, razzias, racisme, liquidations collectives, oppression rationnelle se relayent à des niveaux différents [43] pour littéralement faire de l'autochtone un objet entre les mains de la nation occupante.

Cet homme objet, sans moyens d'exister, sans raison d'être, est brisé, au plus profond de sa substance. Le désir de vivre, de continuer, se fait de plus en plus indécis, de plus en plus fantomatique. C'est à ce stade qu'apparaît le fameux complexe de culpabilité. Wright dans ses premiers romans en donne une description très détaillée.

Progressivement cependant, l'évolution des techniques de production, l'industrialisation, d'ailleurs limitée, des pays asservis, l'existence de plus en plus nécessaire de collaborateurs, imposent à l'occupant une nouvelle attitude. La complexité des moyens de production, l'évolution des rapports économiques entraînant bon gré mal gré celle des idéologies, déséquilibrent le système. Le racisme vulgaire dans sa forme biologique correspond à la période d'exploitation brutale des bras et des jambes de l'homme. La perfection des moyens de production provoque fatalement le camouflage des techniques d'exploitation de l'homme, donc des formes du racisme.

Ce n'est donc pas à la suite d'une évolution des esprits que le racisme perd de sa virulence. Nulle révolution intérieure n'explique cette obligation pour le racisme de se nuancer, d'évoluer. De partout des hommes se libèrent bousculant la léthargie à laquelle oppression et racisme les avaient condamnés.

En plein cœur des «nations civilisatrices» les travailleurs découvrent enfin que l'exploitation de l'homme, base d'un système, emprunte des visages divers. À ce stade le racisme n'ose plus sortir sans fards. Il se conteste. Le raciste dans un nombre de plus en plus grand de circonstances se cache. Celui qui prétendait les « sentir», les « deviner» se découvre visé, regardé, jugé. Le projet du raciste est alors un projet hanté par la mauvaise conscience. Le salut ne peut lui venir que d'un 
engagement passionnel tel qu'on en rencontre dans certaines psychoses. Et ce n'est pas l'un des moindres mérites du Professeur Baruk que d'avoir précisé la sémiologie de ces délires passionnels.

Le racisme n'est jamais un élément surajouté découvert au hasard d'une recherche au sein des données culturelles d'un groupe. La constellation sociale, l'ensemble culturel sont profondément remaniés par l'existence du racisme.

On dit couramment que le racisme est une plaie de l'humanité. Mais il ne faut se satisfaire d'une telle phrase. Il faut [44] inlassablement rechercher les répercussions du racisme à tous les niveaux de sociabilité. L'importance du problème raciste dans la littérature américaine contemporaine est significative. Le nègre au cinéma, le nègre et le folklore, le juif et les histoires pour enfants, le juif au bistrot, sont des thèmes inépuisables.

Le racisme, pour revenir à l'Amérique, haute et vicie la culture américaine. Et cette gangrène dialectique est exacerbée par la prise de conscience et la volonté de lutte de millions de noirs et de juifs visés par ce racisme.

Cette phase passionnelle, irrationnelle, sans justification, présente à l'examen un visage effrayant. La circulation des groupes, la libération, dans certaines parties du monde d'hommes antérieurement infériorisés, rendent de plus en plus précaire l'équilibre. Assez inattendûment le groupe raciste dénonce l'apparition d'un racisme chez les hommes opprimés. Le «primitivisme intellectuel» de la période d'exploitation fait place au « fanatisme moyen-âgeux, voire préhistorique » de la période de libération.

$\grave{A}$ un certain moment on avait pu croire à la disparition du racisme. Cette impression euphorisante, déréelle, était simplement la conséquence de l'évolution des formes d'exploitation. Les psychologues parlent alors d'un préjugé devenu inconscient. La vérité est que la rigueur du système rend superflue l'affirmation quotidienne d'une supériorité. La nécessité de faire appel à des degrés divers à l'adhésion, à la collaboration de l'autochtone modifie les rapports dans un sens moins brutal, plus nuancé, plus « cultivé ». Il n'est d'ailleurs pas rare de voir apparaître à ce stade une idéologie "démocratique et humaine». L'entreprise commerciale d'asservissement, de destruction culturelle cède le pas progressivement à une mystification verbale. 
L'intérêt de cette évolution c'est que le racisme est pris comme thème de méditation, quelquefois même comme technique publicitaire.

C'est ainsi que le blues «plainte des esclaves noirs » est présenté à l'admiration des oppresseurs. C'est un peu d'oppression stylisée qui revient à l'exploitant et au raciste. Sans oppression et sans racisme pas de blues. La fin du racisme sonnerait le glas de la grande musique noire...

Comme dirait le trop célèbre Tomynbee, le blues est une réponse de l'esclave au défi de l'oppression.

[45]

Actuellement encore, pour beaucoup d'hommes, même de couleur, la musique d'Armstrong n'a de véritable sens que dans cette perspective.

Le racisme boursouffle et défigure le visage de la culture qui le pratique. La littérature, les arts plastiques, les chansons pour midinettes, les proverbes, les habitudes, les patterns, soit qu'ils se proposent d'en faire le procès ou de le banaliser, restituent le racisme. C'est dire qu'un groupe social, un pays, une civilisation, ne peuvent être racistes inconsciemment.

Nous le disons encore une fois, le racisme n'est pas une découverte accidentelle. Ce n'est pas un élément caché, dissimulé. Il n'est pas exigé d'efforts surhumains pour le mettre en évidence.

Le racisme crève les yeux car précisément il entre dans un ensemble caractérisé : celui de l'exploitation éhonté d'un groupe d'hommes par un autre parvenu à un stade de développement technique supérieur. C'est pourquoi l'oppression militaire et économique précède la plupart du temps, rend possible, légitime le racisme.

L'habitude de considérer le racisme comme une disposition de l'esprit, comme une tare psychologique doit être abandonnée.

Mais l'homme visé par ce racisme, le groupe social asservi, exploité, désubstantialité, comment se comportent-ils? Quels sont leurs mécanismes de défense?

Quelles attitudes découvrons-nous ici?

Dans une première phase on a vu l'occupant légitimer sa domination par des arguments scientifiques, la « race inférieure » se nier en tant que race. Parce que nulle autre solution ne lui est laissée, le groupe 
social racialisé essaie d'imiter l'oppresseur et par là de se déracialiser. La « race inférieure » se nie en tant que race différente. Elle partage avec la « race supérieure » les convictions, doctrines, et autres attendus la concernant.

Ayant assisté à la liquidation de ses systèmes de référence à l'écroulement de ses schèmes culturels il ne reste plus à l'autochtone qu'à reconnaître avec l'occupant que «Dieu n'est pas de son côté». L'oppresseur, par le caractère global et effrayant de son autorité en arrive à imposer à l'autochtone de nouvelles façons de voir, singulièrement un jugement péjoratif à l'égard de ses formes originales d'exister.

[46]

Cet événement désigné communément aliénation est naturellement très important. On le trouve dans les textes officiels sous le nom d'assimilation.

Or cette aliénation n'est jamais totalement réussie. Soit parce que l'oppresseur quantitativement et qualitativement limite l'évolution, des phénomènes imprévus, hétéroclites, font leur apparition.

Le groupe infériorisé avait admis, la force de raisonnement étant implacable, que ses malheurs procédaient directement de ses caractéristiques raciales et culturelles.

Culpabilité et infériorité sont les conséquences habituelles de cette dialectique. L'opprimé tente alors d'y échapper d'une part en proclamant son adhésion totale et inconditionnelle aux nouveaux modèles culturels, d'autre part en prononçant une condamnation irréversible de son style culturel propre ${ }^{14}$.

Pourtant la nécessité pour l'oppresseur, à un moment donné, de dissimuler les formes d'exploitation, n'entraîne pas la disparition de cette dernière. Les rapports économiques plus élaborés, moins grossiers,

14 Un phénomène, peu étudié apparaît quelquefois à ce stade. Des intellectuels, chercheurs, du groupe dominant étudient «scientifiquement» la société dominée, son esthétique, son univers éthique.

Dans les Universités les rares intellectuels colonisés se voient révéler leur système culturel. Il arrive même que les savants des pays colonisateurs s'enthousiasment pour tel trait spécifique. Les concepts de pureté, naïveté, innocence apparaissent. La vigilance de l'intellectuel indigène doit redoubler ici. 
exigent un revêtement quotidien mais l'aliénation à ce niveau demeure épouvantable.

Ayant jugé, condamné, abandonné ses formes culturelles, son langage, son alimentation, ses démarches sexuelles, sa façon de s'asseoir, de se reposer, de rire, de se divertir, l'opprimé, avec l'énergie et la ténacité du naufragé se rue sur la culture imposée.

Développant ses connaissances techniques au contact de machines de plus en plus perfectionnées, entrant dans le circuit dynamique de la production industrielle, rencontrant des hommes de régions éloignées dans le cadre de la concentration des capitaux donc des lieux de travail, découvrant la chaîne, l'équipe, le « temps » de production, c'est-àdire le rendement à l'heure, l'opprimé constate comme un scandale, le maintien à son égard, du racisme et du mépris.

[47]

C'est à ce niveau que l'on fait du racisme une histoire de personnes. «Il existe quelques racistes indécrottables mais avouez que dans l'ensemble la population aime... »

Avec le temps tout cela disparaîtra.

Ce pays est le moins raciste...

Il existe à l'O.N.U. une commission chargée de lutter contre le racisme.

Des films sur le racisme, des poèmes sur le racisme, des messages sur le racisme...

Les condamnations spectaculaires et inutiles du racisme. La réalité est qu'un pays colonial est un pays raciste. Si en Angleterre, en Belgique ou en France, en dépit des principes démocratiques affirmés par ces nations respectives, il se trouve encore des racistes, ce sont ces racistes qui, contre l'ensemble du pays, ont raison.

Il n'est pas possible d'asservir des hommes sans logiquement les inférioriser de part en part. Et le racisme n'est que l'explication émotionnelle, affective, quelquefois intellectuelle de cette infériorisation.

Le raciste dans une culture avec racisme est donc normal. L'adéquation des rapports économiques et de l'idéologie est chez lui parfaite. Certes l'idée que l'on se fait de l'homme n'est jamais totalement dépendante des rapports économiques c'est-à-dire, ne l'oublions 
pas, des rapports existant historiquement et géographiquement entre les hommes et les groupes. Des membres de plus en plus nombreux appartenant à des sociétés racistes prennent position. Ils mettent leur vie au service d'un monde où le racisme serait impossible. Mais ce recul, cette abstraction cet engagement solennel ne sont pas à la portée de tous. On ne peut exiger sans dommage qu'un homme soit contre les «préjugés de son groupe ».

Or, redisons-le, tout groupe colonialiste est raciste.

À la fois «acculturé » et déculturé l'opprimé continue à buter contre le racisme. Il trouve illogique cette séquelle. Inexplicable ce qu'il a dépassé, sans motif, inexact. Ses connaissances, l'appropriation de techniques précises et compliquées, quelquefois sa supériorité intellectuelle eu égard à un grand nombre de racistes, l'amènent à qualifier le monde raciste de passionnel. Il s'aperçoit que l'atmosphère raciste imprègne tous les éléments de la vie sociale. Le sentiment d'une injustice accablante est alors très vif. Oubliant le racismeconséquence on s'acharne sur le racisme-cause. Des campagnes de [48]

désintoxication sont entreprises. On fait appel au sens de l'humain, à l'amour, au respect des valeurs suprêmes...

En fait le racisme obéit à une logique sans faille. Un pays qui vit, tire sa substance de l'exploitation de peuples différents, infériorise ces peuples. Le racisme appliqué à ces peuples est normal.

Le racisme n'est donc pas une constante de l'esprit humain.

Il est, nous l'avons vu, une disposition inscrite dans un système déterminé. Et le racisme juif n'est pas différent du racisme noir. Une société est raciste ou ne l'est pas. Il n'existe pas de degrés du racisme. Il ne faut pas dire que tel pays est raciste mais qu'on n'y trouve pas de lynchages ou de camps d'extermination. La vérité est que tout cela et autre chose existe en horizon. Ces virtualités, ces latences circulent dynamiques, prises dans la vie des relations psycho-affectives, économiques...

Découvrant l'inutilité de son aliénation, l'approfondissement de son dépouillement, l'infériorisé, après cette phase de déculturation, d'extranéisation, retrouve ses positions originales. 
Cette culture, abandonnée, quittée, rejetée, méprisée, l'infériorisé s'y engage avec passion. Il existe une surenchère très nette s'apparentant psychologiquement au désir de se faire pardonner.

Mais derrière cette analyse simplifiante il y a bel et bien l'intuition par l'infériorisé d'une vérité spontanée apparue. Cette histoire psychologique débouche sur l'Histoire et sur la Vérité.

Parce que l'infériorisé retrouve un style autrefois dévalorisé on assiste à une culture de la culture. Une telle caricature de l'existence culturelle signifierait s'il en était besoin que la culture se vit mais ne se morcelle pas. Elle ne se met pas entre lame et lamelle.

Cependant l'opprimé s'extasie à chaque redécouverte. L'émerveillement est permanent. Autrefois émigré de sa culture, l'autochtone l'explore aujourd'hui avec fougue. Il s'agit alors d'épousailles continuées. L'ancien infériorisé est en état de grâce.

Or, on ne subit pas impunément une domination. La culture du peuple asservi est sclérosée, agonisante. Aucune vie n'y circule. Plus précisément la seule vie existante est dissimulée. La population qui normalement assume çà et là quelques [49] morceaux de vie, qui maintient des significatives dynamiques aux institutions est une population anonyme. En régime colonial ce sont les traditionalistes.

L'ancien émigré, par l'ambigüité soudaine de son comportement introduit le scandale. À l'anonymat du traditionaliste il oppose un exhibitionnisme véhément et agressif.

État de grâce et agressivité sont deux constantes retrouvées à ce stade. L'agressivité étant le mécanisme passionnel permettant d'échapper à la morsure du paradoxe.

Parce que l'ancien émigré possède des techniques précises, parce que son niveau d'action se situe dans le cadre de rapports déjà complexes, ces retrouvailles revêtent un aspect irrationnel. Il existe un fossé, un écart entre le développement intellectuel, l'appropriation technique, les modalités de pensée et de logique hautement différenciées et une base émotionnelle « simple, pure », etc...

Retrouvant la tradition, la vivant comme mécanisme de défense, comme symbole de pureté, comme salut, le déculturé laisse l'impression que la médiation se venge en se substantialisant. Ce reflux sur des positions archaiques sans rapport avec le développement tech- 
nique est paradoxal. Les institutions ainsi valorisées ne correspondent plus aux méthodes élaborées d'action déjà acquises.

La culture encapsulée, végétative, depuis la domination étrangère est revalorisée. Elle n'est pas repensée, reprise, dynamisée de l'intérieur. Elle est clamée. Et cette revalorisation d'emblée, non structurée, verbale, recouvre des attitudes paradoxales.

C'est à ce moment qu'il est fait mention du caractère indécrottable des infériorisés. Les médecins arabes dorment par terre, crachent n'importe où, etc...

Les intellectuels noirs consultent le sorcier avant de prendre une décision, etc...

Les intellectuels «collaborateurs » cherchent à justifier leur nouvelle attitude. Les coutumes, traditions, croyances. autrefois niées et passées sous silence sont violemment valorisées et affirmées.

La tradition n'est plus ironisée par le groupe. Le groupe ne se fuit plus. On retrouve le sens du passé, le culte des ancêtres...

Le passé, désormais constellation de valeurs, s'identifie à la Vérité.

[50]

Cette redécouverte, cette valorisation absolue d'allure quasi déréelle, objectivement indéfendable, revêt une importance subjective incomparable. Au sortir de ces épousailles passionnées, l'autochtone aura décidé, en « connaissance de cause», de lutter contre toutes les formes d'exploitation et d'aliénation de l'homme. Par contre l'occupant à cette époque multiplie les appels à l'assimilation, puis à l'intégration, à la communauté.

Le corps à corps de l'indigène avec sa culture est une opération trop solennelle, trop abrupte, pour tolérer une quelconque faille. Nul néologisme ne peut masquer la nouvelle évidence: la plongée dans le gouffre du passé est condition et source de liberté.

La fin logique de cette volonté de lutte est la libération totale du territoire national. Afin de réaliser cette libération l'infériorisé met en jeu toutes ses ressources, toutes ses acquisitions, les anciennes et les nouvelles, les siennes et celles de l'occupant.

La lutte est d'emblée totale, absolue. Mais alors, on ne voit guère apparaître de racisme. 
Au moment d'imposer sa domination, pour justifier l'esclavage, l'oppresseur avait fait appel à des argumentations scientifiques. Ici rien de pareil.

Un peuple qui entreprend une lutte de libération, légitime rarement le racisme. Même au cours des périodes aiguës de lutte armée insurrectionnelle on n'assiste jamais à la prise en masse de justifications biologiques.

La lutte de l'infériorisé se situe à un niveau nettement plus humain. Les perspectives sont radicalement nouvelles. C'est l'opposition désormais classique des luttes de conquête et de libération.

En cours de lutte la nation dominatrice essaie de rééditer des arguments racistes mais l'élaboration du racisme se révèle de plus en plus inefficace. On parle de fanatisme, d'attitudes primitives en face de la mort mais encore une fois, le mécanisme désormais effondré, ne répond plus. Les anciens immobiles, les lâches constitutionnels, les peureux, les infériorises de toujours s'arc-boutent et émergent hérissés.

L'occupant ne comprends plus.

La fin du racisme commence avec une soudaine incompréhension.

[51]

La culture spasmée et rigide de l'occupant, libérée s'ouvre enfin à la culture du peuple devenu réellement frère. Les deux cultures peuvent s'affronter, s'enrichir.

En conclusion, l'universalité réside dans cette décision de prise en charge du relativisme réciproque de cultures différentes une fois exclu irréversiblement le statut colonial. 
[53]

Pour la révolution africaine Écrits politiques

Troisième partie

Pour l'Algérie

Retour à la table des matières 
[55]

Pour la révolution africaine

Écrits politiques

Troisième partie. Pour l'Algérie.

Chapitre 1

Lettre à un Français

Retour à la table des matières

Quand tu m'as dit ton désir de quitter l'Algérie, mon amitié soudain s'est faite silencieuse. Certes des images surgies, tenaces et décisives étaient à l'entrée de ma mémoire.

Je te regardais et ta femme à côté.

Tu te voyais déjà en France... De nouveaux visages autour de toi, très loin de ce pays où depuis quelques jours les choses décidément ne vont pas bien.

Tu m'as dit, l'atmosphère se gâte, il faut que je m'en aille. Ta décision sans être irrévocable parce que tu l'avais exprimée, progressivement prenait forme. 
Ce pays inexplicablement hérissé! Les routes qui ne sont plus sûres. Les champs de blé transformés en brasiers. Les Arabes qui se font méchants.

On raconte. On raconte.

Les femmes seront violées. Les testicules seront coupés et fichés entre les dents.

Rappelez-vous Sétif! Voulez-vous un autre Sétif?

Ils l'auront mais pas nous.

Tu m'as dit tout cela en riant.

Mais ta femme ne riait pas.

Et derrière ton rire j'ai vu.

J'ai vu ton essentielle ignorance des choses de ce pays.

Des choses car je t'expliquerai.

Peut-être partiras-tu, mais dis-moi, quand on te demandera: «Que se passe-t-il en Algérie? » Que répondras-tu?

Quand tes frères te demanderont: qu'est-il arrivé en Algérie? Que leur répondras-tu?

Plus précisément quand on voudra comprendre pourquoi [56] tu as quitté ce pays, comment feras-tu pour éteindre cette honte que déjà tu traînes?

Cette honte (de n'avoir pas compris, de n'avoir pas voulu comprendre ce qui autour de toi s'est passé tous les jours.

Huit ans durant tu fus dans ce pays.

Et pas un morceau de cette énorme plaie qui t'ait empêché !

Et pas un morceau de cette énorme plaie qui t'ait obligé !

De te découvrir enfin tel.

Inquiet de l'Homme mais singulièrement pas de l'Arabe. Soucieux, angoissé, tenaillé.

Mais en plein champ, ton immersion dans la même boue, dans la même lèpre. 
Car pas un Européen qui ne se révolte, ne s'indigne, ne s'alarme de tout, sauf du sort fait à l'Arabe.

Arabes inaperçus.

Arabes ignorés.

Arabes passés sous silence.

Arabes subtilisés, dissimulés.

Arabes quotidiennement niés, transformés en décor saharien. Et toi mêlé à ceux :

Qui n'ont jamais serré la main à un Arabe.

Jamais bu le café.

Jamis parlé du temps qu'il fait avec un Arabe.

À tes côtés les Arabes.

Écartés les Arabes.

Sans effort rejetés les Arabes.

Confinés les Arabes.

Ville indigène écrasée.

Ville d'indigènes endormis.

Il n'arrive jamais rien chez les Arabes.

Toute cette lèpre sur ton corps.

Tu partiras. Mais toutes ces questions, ces questions sans réponse. Le silence conjugué de 800.000 Français, ce silence ignorant, ce silence innocent.

Et 9.000.000 d'hommes sous ce linceul de silence.

Je t'offre ce dossier afin que nul ne meure, ni les morts d'hier, ni les ressucités d'aujourd'hui.

Je veux ma voix brutale, je ne la veux pas belle, je ne la veux pas pure, je ne la veux pas de toutes dimensions.

Je la veux de part en part déchirée, je ne veux qu'elle s'amuse car enfin, je parle de l'homme et de son refus, de la [57] quotidienne pourriture de l'homme, de son épouvantable démission.

Je veux que tu racontes. 
Que je dise par exemple: il existe une crise de la scolarisation en Algérie, pour que tu penses : c'est dommage il faut y remédier.

Que je dise: un Arabe sur trois cents qui sache signer son nom, pour que tu penses: c'est triste, il faut que cela cesse.

Écoute plus avant :

Une directrice d'école se plaignant devant moi, se plaignant à moi d'être obligée chaque année d'admettre dans son école de nouveaux petits Arabes.

Une directrice d'école se plaignant, une fois tous les Européens inscrits, d'être obligée de scolariser quelques petits Arabes.

L'analphabétisme de ces petits bicots qui croît à la mesure même de notre silence.

Instruire les Arabes, mais vous n'y pensez pas.

Vous voulez donc nous compliquer la vie.

Ils sont bien comme ils sont.

Moins ils comprennent, mieux cela vaut.

Et où prendre les crédits.

Cela va vous coûter les deux yeux de la tête.

D'ailleurs ils n'en demandent pas tant.

Une enquête faite auprès des Caïds montre que l'Arabe ne réclame pas d'écoles.

Millions de petits cireurs. Millions de « porter madame».

Millions de donne-moi un morceau de pain. Millions d'illettrés «ne sachant pas signer, ne signe, signons ».

Millions d'empreintes digitales sur les procès-verbaux qui conduisent en prisons.

Sur les actes de Monsieur le Cadi.

Sur les engagements dans les régiments de tirailleurs algériens.

Millions de fellahs exploités, trompés, volés.

Fellahs agrippés à quatre heures du matin, abandonnés à huit heures du soir. Du soleil à la lune. 
Fellahs gorgés d'eau, gorgés de feuilles, gorgés de vieille galette qui doit faire tout le mois.

Fellah immobile et tes bras bougent et ton dos courbé mais ta vie arrêtée.

[58]

Les voitures passent et vous ne bougez pas. On vous passerait sur le ventre que vous ne bougeriez pas.

Arabes sur les routes.

Bâtons passés dans l'anse du panier.

Panier vide, espoir vide, toute cette mort du fellah.

Deux cent cinquante francs par jour.

Fellah sans terre.

Fellah sans raison.

Si vous n'êtes pas contents vous n'avez qu'à partir. Des enfants pleins la case. Des femmes pleines dans les cases.

Fellah essoré.

Sans rêve.

Six fois deux cent cinquante francs par jour.

Et rien ici ne vous appartient.

On est gentil avec vous, de quoi vous plaignez-vous?

Sans nous que feriez-vous? Ah, il serait joli ce pays si nous nous en allions?

Transformé en marais au bout de peu de temps, oui !

Vingt-quatre fois deux cent cinquante francs par jour.

Travaille fellah. Dans ton sang l'éreintement prosterné de toute une vie.

Six mille francs par mois. 
Sur ton visage le désespoir.

Dans ton ventre la résignation...

Qu'importe fellah si ce pays est beau. 
[59]

\author{
Pour la révolution africaine \\ Écrits politiques
}

Troisième partie. Pour l'Algérie.

Chapitre 2

\title{
Lettre au Ministre Résident 1956
}

Retour à la table des matières

\author{
Monsieur le Docteur Frantz FANON \\ Médecin des Hôpitaux Psychiatriques \\ Médecin-Chef de service à \\ l'Hôpital Psychiatrique de \\ BLIDA-JOINVILLE \\ à Monsieur le Ministre Résident, \\ Gouverneur Général de l'Algérie
}

ALGER

Monsieur le Ministre,

Sur ma demande et par arrêté en date du 22 octobre 1953, Monsieur le Ministre de la Santé Publique et de la Population a bien voulu me mettre à la disposition de Monsieur le Gouverneur Général de l'Algérie pour être affecté à un Hôpital Psychiatrique de l'Algérie. 
Installé, à l'Hôpital Psychiatrique de Blida-Joinville le 23 novembre 1953, j'y exerce depuis cette date les fonctions de Médecin-Chef de service.

Bien que les conditions objectives de la pratique psychiatrique en Algérie fussent déjà un défi au bon sens, il m'était apparu que des efforts devaient être entrepris pour rendre moins vicieux un système dont les bases doctrinales s'opposaient quotidiennement à une perspective humaine authentique.

[60]

Pendant près de trois ans je me suis mis totalement au service de ce pays et des hommes qui l'habitent. Je n'ai ménagé ni mes efforts, ni mon enthousiasme. Pas un morceau de mon action qui n'ait exigé comme horizon l'émergence unanimement souhaitée d'un monde valable.

Mais que sont l'enthousiasme et le souci de l'homme si journellement la réalité est tissée de mensonges, de lâchetés, du mépris de l'homme?

Que sont les intentions si leur incarnation est rendue impossible par l'indigence du cœur, la stérilité de l'esprit, la haine des autochtones de ce pays?

La Folie est l'un des moyens qu'a l'homme de perdre sa liberté. Et je puis dire, que placé à cette intersection, j'ai mesuré avec effroi l'ampleur de l'aliénation des habitants de ce pays.

Si la psychiatrie est la technique médicale qui se propose de permettre à l'homme de ne plus être étranger à son environnement, je me dois d'affirmer que l'Arabe, aliéné permanent dans son pays, vit dans un état de dépersonnalisation absolue.

Le statut de l'Algérie? Une déshumanisation systématisée.

Or le pari absurde était de vouloir coûte que coûte faire exister quelques valeurs alors que le non-droit, l'inégalité, le meurtre multiquotidien de l'homme étaient érigés en principes législatifs.

La structure sociale existant en Algérie s'opposait à toute tentative de remettre l'individu à sa place.

Monsieur le Ministre, il arrive un moment où la ténacité devient persévération morbide. L'espoir n'est plus alors la porte ouverte sur 
l'avenir mais le maintien illogique d'une attitude subjective en rupture organisée avec le réel.

Monsieur le Ministre, les événements actuels qui ensanglantent l'Algérie ne constituent pas aux yeux de l'observateur un scandale. Ce n'est ni un accident, ni une panne du mécanisme.

[61]

Les événements d'Algérie sont la conséquence logique d'une tentative avortée de décérébraliser un peuple.

Il n'était point exigé d'être psychologue pour deviner sous la bonhomie apparente de l'Algérien, derrière son humilité dépouillée, une exigence fondamentale de dignité. Et rien ne sert, à l'occasion de manifestations non simplifiables, de faire appel à un quelconque civisme.

La fonction d'une structure sociale est de mettre en place des institutions traversées par le souci de l'homme. Une société qui accule ses membres à des solutions de désespoir est une société non viable, une société à remplacer.

Le devoir du citoyen est de le dire. Aucune morale professionnelle, aucune solidarité de classe, aucun désir de laver le linge en famille ne prévaut ici. Nulle mystification pseudo-nationale ne trouve grâce devant l'exigence de la pensée.

Monsieur le Ministre, la décision de sanctionner les grévistes du 5 juillet 1956 est une mesure qui, littéralement, me paraît irrationnelle.

Ou les grévistes ont été terrorisés dans leur chair et celle de leur famille, alors il fallait comprendre leur attitude, la juger normale, compte tenu de l'atmosphère.

Ou leur abstention traduisait un courant d'opinion unanime, une conviction inébranlable, alors toute attitude sanctionniste était superflue, gratuite, inopérante.

Je dois à la vérité de dire que la peur ne m'a pas paru être le trait dominant des grévistes. Bien plutôt il y avait le vou inéluctable de susciter dans le calme et le silence une ère nouvelle toute de paix et de dignité.

Le travailleur dans la cité doit collaborer à la manifestation sociale. Mais il faut qu'il soit convaincu de l'excellence de cette société vécue. Il arrive un moment où le silence devient mensonge. 
Les intentions maîtresses de l'existence personnelle s'accommodent mal des atteintes permanentes aux valeurs les plus banales.

Depuis de longs mois ma conscience est le siège de débats impardonnables. Et leur conclusion est la volonté de ne pas désespérer de I'homme, c'est-à-dire de moi-même.

Ma décision est de ne pas assurer une responsabilité coûte [62] que coûte, sous le fallacieux prétexte qu'il n'y a rien d'autre à faire.

Pour toutes ces raisons, j'ai l'honneur, Monsieur le Ministre, de vous demander de bien vouloir accepter ma démission et de mettre fin à ma mission en Algérie, avec l'assurance de ma considération distinguée. 
[63]

Pour la révolution africaine

Écrits politiques

Quatrième partie

Vers la libération

de l'Afrique 


\author{
Pour la révolution africaine \\ Écrits politiques
}

Quatrième partie. Vers la libération de l'Afrique.

\title{
Chapitre 1 \\ Déceptions et illusions du colonialisme français 15
}

\section{Retour à la table des matières}

Depuis vingt ans les peuples coloniaux disloquent la domination étrangère et prennent pied sur la scène internationale. Les unes après les autres et à des allures différentes, les vieilles métropoles se retirent de leurs possessions. Si les expéditions coloniales obéissent à un schéma donné et connu - nécessité de faire régner l'ordre chez les barbares, protection des concessions et intérêts des pays européens, apport généreux de la civilisation occidentale -, on n'a pas suffisam-

El Moudjahid, $N^{0} 10$, septembre 1957. En règle générale, dans cette série d'articles de l'organe central du F.L.N., les événements auxquels se réfère F. Fanon sont suffisamment présent encore dans toutes les mémoires, et l'auteur luimême est allé suffisamment loin dans son souci de clarté et de didactisme, pour que l'on n'ait pas jugé utile d'alourdir ces textes de notices historiques explicatives. 
ment montré la stéréotypie des moyens utilisés par les métropoles pour s'accrocher à leurs colonies.

La guerre franco-algérienne, par ses proportions et par son acuité, permet de voir en gros plan, à cause même de leur échec successif, les tentatives faites par la France pour maintenir sa domination.

\section{L'impossible collaboration}

La première tactique des pays colonialistes consiste à s'appuyer sur les collaborateurs officiels et les féodaux. Les Algériens, [66] qu'une série de compromissions désigne particulièrement, sont regroupés, et priés de condamner publiquement « ce mouvement séditieux qui trouble la paix de la cité ». En 1954 et au cours des premiers mois de 1955, la France procède au recensement et à la mobilisation de ses fidèles et loyaux serviteurs. Déclaration, condamnations, appels à la sagesse sont rédigés, publiée ou lus à la radio.

Les autorités colonialistes attendent avec confiance, puis avec anxiété, enfin sans espoir les résultats de ces messages. Sollicités à nouveau, les serviteurs prennent l'habitude jusqu'alors inconnue de décliner les invitations, fuient les mises en scènes officielles et adoptent souvent un vocabulaire nouveau.

C'est que l'engagement révolutionnaire se révèle de plus en plus total et les collaborateurs ont conscience du gigantesque réveil d'un peuple en armes.

\section{L'argument économique}

Devant la défection d'hommes qu'elle avait pourtant utilisés et déshonorés aux yeux du peuple algérien et devant l'hostilité active des élites, les autorités françaises lancent la deuxième opération.

Elle se ramène fondamentalement à couper la population prétendue «saine » du mouvement révolutionnaire. 
Incapable d'appréhender la signification réelle du combat libérateur, la France, dans un premier temps, reconnaît l'existence d'un problème qu'elle déclare économique et social. Dans l'espoir de faire taire la voix de la dignité nationale, elle « s'engage solennellement à combattre la misère et à régler les problèmes du logement». Les salaires sont symboliquement augmentés et des programmes d'investissement annoncés. Cette assimilation d'une revendication nationale à une jacquerie ou à un mécontentement social obéit à une double mystification: il n'existe pas de conscience nationale algérienne et les promesses d'amélioration du niveau de vie des populations doivent suffire à ramener l'ordre et la paix.

Mais les autorités françaises, utilisant des indicateurs de plus en plus rares et de plus en plus coûteux, apprennent avec un certain désarroi que le mouvement est solide, enraciné dans la masse et animé par elle.

[67]

\section{Contre un front uni, des méthodes inhumaines et cyniques}

Dans un deuxième temps, et avec une rare duplicité, l'administration française organise l'opération Mozabites, l'opération Kabyles, l'opération Juifs, l'opération Harkas. Ce qui est concrètement recherché, c'est l'apparition au sein de la population de courants intérieurs contradictoires, donc contre-révolutionnaires. L'exploitation d'un certain nombre d'hostilités locales créées par le colonialisme, l'entretien et l'intensification provoquée de différences culturelles transformées en lutte de clans ou, quelquefois, de « races » caractérisent ces opérations.

Mélouza et Wagram portent à un point ultime de cruauté des méthodes où viols, massacres ostensiblement signés F.L.N. ratissages de douars entiers ont pour but de provoquer la révolte de la population et la condamnation du mouvement révolutionnaire. La commune erreur de ces différentes mancuures réside dans le fait que les autorités françaises oublient singulièrement que le F.L.N. s'identifie au peuple algé- 
rien. Les maris des femmes violées se trouvaient dans le groupe F.L.N. local. Le soir, ils descendaient de leurs secteurs opérationnels pour embrasser leurs enfants. Et les maisons du douar détruit avaient été construites par les moudjahidines qui tenaient la montagne environnante.

L'état-major, victime d'une politique inactuelle et de son absence de renseignements sur la structure du F.L.N., s'imagine que dans les montagnes tout peut se passer.

Or, rien ne se passe qui ne soit prévu et décidé.

Les déplacements des groupes obéissent à un programme stratégique fixé par l'état-major de l'A.L.N. Chaque unité a un secteur précis et un P.C. qui la coordonne.

Il n'existe pas d'unité du F.L.N. en migration plus ou moins cohérente qui pourrait massacrer ici ou là. Quand une compagnie ou un bataillon se déplace en dehors de son secteur ou de sa région, c'est à la suite d'un ordre de l'état-major de Wilaya. Communication en est auparavant donnée aux différents P.C. régionaux ou zonaux, et la progression est couverte par les unités locales.

Parce qu'elles ignoraient cela, les autorités françaises ont lâché leurs soldats et leurs harkas sur les populations civiles algériennes.

[68]

Chaque fois, la volonté d'indépendance s'est faite plus irréductible.

L'opération Mozabites dura peu de jours. Commerçants pour la plupart, ces Algériens reçurent de nombreuses lettres de menaces. Des raids furent entrepris sur leurs magasins. Une atmosphère d'allure raciste fut déclenchée. Cette tentative grossière devait assez rapidement échouer à la suite d'une mise au point du F.L.N.

L'opération Juifs se situait pareillement dans une perspective raciste. Elle devait être dénoncée dans la célèbre lettre du F.L.N. à la communauté juive d'Algérie.

Toutefois la carte maîtresse du colonialisme était représentée par le M.N.A. Inexistant sur le territoire national, le messalisme bénéficiait en France de l'appui inconditionnel de l'ennemi. Les Français ont à plusieurs reprises facilité le transport de centaines de messalistes et procédé à leur armement. Identifiés rapidement à leur arrivée sur le 
territoire national par le service de renseignements du F.L.N., ils rejoignaient nos rangs ou étaient condamnés à mort et exécutés pour trahison envers la cause nationale et collaboration avec l'ennemi.

\section{Une explication classique}

Il ne restait plus à la France qu'une troisième et dernière opération à mener. Ses deux moments sont généralement jumelés: découverte d'une inspiration étrangère et particulièrement communiste des mouvements de libération nationale.

La première phase, spectaculaire, illustre parfaitement le degré d'inconscience atteint par les gouvernements français. L'expédition de Suez se proposait de frapper la Révolution algérienne au sommet. L'Egypte, accusée de diriger la lutte du peuple algérien, était criminellement bombardée. La paix internationale, un moment en danger, devait être sauvée par l'attitude vigoureuse et sans équivoque de l'Organisation des Nations Unies.

Or, dans le même temps, les opérations militaires s'intensifient en Algérie. Le F.L.N. prend l'initiative sur toute l'étendue du territoire. La grande grève de 8 jours réaffirme l'unanimité nationale dans la lutte et le maintien des objectifs.

La deuxième phase commencée, abandonnée et reprise ne fut jamais menée à son terme. L'épouvantail communiste fut [69] peu exploité. Confusément, les colonialistes français en sentaient l'incohérence. Ils n'étaient pas convaincus par cette thèse.

Trois opérations politiques qui se sont heurtées, comme les opérations militaires parallèles, aux forces nationales algériennes. Toutes les méthodes connues, toutes les mancuvres habituelles se sont révélées inefficaces, inadéquates, inutiles. Certes, épisodiquement l'une ou l'autre de ces opérations est relancée. Mais le ressort est cassé. 


\section{Des rêves insensés}

Face au peuple algérien, les stratèges français ne comprennent plus. Leurs schémas classiques et longuement éprouvés sont désormais inutilisables.

Aussi voit-on depuis quelques mois la France sombrer dans un probabilisme caractérisé. Les déclarations de ses hommes politiques empruntent fréquemment l'allure prophétique.

Des différends seraient sur le point de se faire jour au sein du F.L.N. Les militaires essaieraient de prendre la direction du mouvement. Une lutte interne très dure existerait entre extrémistes et modérés. Les Kabyles feraient prochainement un coup d'Etat. Enfin, une lutte des colonels entre eux serait imminente.

Abandonnant l'action et fuyant les décisions réalistes, la France en Algérie espère, souhaite et prophétise.

Isolée sur le territoire national, sans aucun contact avec le peuple algérien, la France adopte des positions de moins en moins concrètes, de plus en plus illusoires.

Normalement, pensent les gouvernants français, les Algériens devraient commencer à se fatiguer.

On formule des vœux, on émet des hypothèses et selon une logique bien connue, on les transforme en éléments du réel : les membres du Conseil National de la Révolution Algérienne sont divisés et les méchants militaires terroriseraient les partisans de la négociation. Quelquefois, déçus par l'inefficacité de leurs désirs, les Français boudent.

Au F.L.N., on reproche son caractère monolithique, son absence de fissure et au peuple algérien, de se battre pour un mort.

Or, l'affrontement du réel exige d'autres techniques. Les autorités françaises doivent se rendre compte une fois pour [70] toutes que l'on n'échappe pas aux faits. Le refuge dans le monde des désirs, dans des colères inutiles ne constitue pas une solution à la guerre francoalgérienne. 
Oui, le peuple algérien depuis trois ans est monolithique. C'est que le mot d'ordre est d'une clarté et d'une simplicité inhabituelles.

Indépendance nationale par la lutte armée, objectifs, limites, méthodes et moyens de la lutte sont précisés une fois pour toutes.

La chimère de dissensions éventuelles manifeste une totale absence de sens critique puisqu'aussi bien la réalité ne semble pas vouloir se conformer à ces visions ou à ces désirs.

Le F.L.N. n'est pas un mouvement de revendications professionnelles et tout marchandage est impensable.

Le C.N.R.A. ne représente pas un groupe d'intérêts, mais l'étatmajor politico-militaire d'une Nation en lutte pour son indépendance.

Sans prises sur le réel, incapables on refusant de reconnaître la volonté nationale algérienne et de tirer les conclusions logiques qui s'imposent, les autorités françaises vivent aujourd'hui sous le signe des désirs et des prophéties. 


\section{Pour la révolution africaine \\ Écrits politiques}

Quatrième partie. Vers la libération de l'Afrique.

\section{Chapitre 2 \\ L'Algérie face aux tortionnaires français 16}

\section{Retour à la table des matières}

La Révolution algérienne, par l'inspiration profondément humaine qui l'anime et son culte passionné de la liberté, procède depuis trois ans à la destruction méthodique d'un certain nombre de mystifications.

Certes, la Révolution algérienne restitue ses droits à l'existence nationale. Certes, elle témoigne de la volonté du peuple. Mais l'intérêt et la valeur de notre Révolution résident dans le message dont elle est porteuse.

Les pratiques authentiquement monstrueuses qui sont apparues depuis le $1^{\text {er }}$ novembre 1954 étonnent surtout par leur généralisation... En réalité, l'attitude des troupes françaises en Algérie se situe dans une structure de domination policière, de racisme systématique, de dés-

16 El Moudjahid, N¹0, septembre 1957. 
humanisation poursuivie de façon rationnelle. La torture est inhérente à l'ensemble colonialiste.

La Révolution algérienne, en se proposant la libération du territoire national, vise, et la mort de cet ensemble, et l'élaboration d'une société nouvelle. L'indépendance de l'Algérie n'est pas seulement fin du colonialisme mais disparition, dans cette partie du monde, d'un germe de gangrène et d'une source d'épidémie.

La libération du territoire national algérien est une défaite pour le racisme et l'exploitation de l'homme; elle inaugure le règne inconditionnel de la Justice.

[72]

\section{La véritable contradiction}

Les guerres de libération nationale sont souvent présentées comme exprimant les contradictions internes des pays colonialistes. La guerre franco-algérienne, bien que s'inscrivant dans un contexte historique caractérisé par l'éclosion simultanée et successive de mouvements de libération nationale, présente des particularités propres.

Colonie de peuplement déclarée territoire métropolitain, l'Algérie a vécu sous une domination policière et militaire jamais égalée en pays colonial. Ceci s'explique d'abord par le fait que l'Algérie n'a pratiquement jamais déposé les armes depuis 1830. Mais surtout, la France n'ignore pas l'importance de l'Algérie dans son dispositif colonial, et rien ne peut expliquer son obstination et ses incalculables efforts, sinon la certitude que l'indépendance de l'Algérie entraînera à brève échéance l'écroulement de son empire.

L'Algérie, située aux portes de la France, permet au monde occidental de voir dans le détail et comme au ralenti les contradictions de la situation coloniale.

L'appel au contingent français, la mobilisation de plusieurs classes, le rappel des officiers et des sous-officiers, les invitations au sacrifice lancées périodiquement au peuple, les impôts et le blocage des salaires ont engagé la totalité de la Nation française dans cette guerre de reconquête coloniale. 
L'enthousiasme généralisé, et quelquefois véritablement sanguinaire, qui a marqué la participation des ouvriers et des paysans français à la guerre contre le peuple algérien a ébranlé dans ses fondements la thèse d'un pays réel qui s'opposerait au pays légal.

Selon une phrase significative d'un des Présidents du Conseil français, la Nation s'est identifiée avec son armée qui se bat en Algérie.

La guerre d'Algérie est faite consciencieusement par tous les Français et les quelques critiques exprimées jusqu'ici par quelques individualistes évoquent uniquement certaines méthodes qui « précipitent la perte de l'Algérie». Mais la reconquête coloniale dans son essence, l'expédition armée, la tentative d'étouffer la liberté, d'un peuple ne sont pas condamnées.

[73]

\section{La torture, nécessité fondamentale du monde colonial}

Depuis quelques temps on parle beaucoup de tortures appliquées par les soldats français aux patriotes algériens. Des textes abondants, précis, effroyables, ont été publiés. Des comparaisons historiques ont été faites. Des personnalités étrangères, et parmi elles des Français, ont condamné ces pratiques.

Les Français qui s'insurgent contre la torture, ou en déplorent l'extension, font immanquablement penser à ces belles âmes dont parlait tel philosophe, et l'appellation « d'intellectuels fatigués » qui leur est donnée par leurs compatriotes Lacoste et Lejeune est très pertinente. On ne peut à la fois vouloir le maintien de la domination française en Algérie et condamner les moyens de ce maintien.

La torture en Algérie n'est pas un accident, ou une erreur, ou une faute. Le colonialisme ne se comprend pas sans la possibilité de torturer, de violer ou de massacrer.

La torture est une modalité des relations occupant-occupé.

Les policiers français, qui pendant longtemps ont été les seuls à pratiquer ces tortures, ne l'ignorent pas. La nécessité de légitimer les 
tortures a toujours été considérée par eux comme un scandale et un paradoxe.

\section{La torture, style de vie}

Il reste que le système a des accidents, des pannes. Leur analyse est d'une extrême importance.

Au cours du premier trimestre 1956, des cas de policiers à la limite de la folie se sont révélés nombreux.

Les troubles qu'ils présentaient au sein du milieu familial (menaces de mort adressées à leur femme, sévices graves sur leurs enfants, insomnies, cauchemars, menaces continuelles de suicide 17 et les fautes professionnelles dont ils se sont rendus coupables (rixes avec des Collègues, laisser-aller dans le service, [74] manque d'énergie, attitudes irrespectueuses avec leurs chefs) ont nécessité à maintes reprises des soins médicaux, l'affectation dans un autre service ou, plus souvent, une mutation en France.

L'apparition multiple d'organismes révolutionnaires dynamiques, les réactions foudroyantes de nos fidayines, l'implantation du F.L.N. sur l'ensemble du territoire national posaient aux policiers français des problèmes insurmontables. Le qui-vive permanent auquel les condamnait le F.L.N. semblait devoir expliquer l'irritabilité, des policiers.

Or, rapidement, les policiers s'expliquent.

Ils frappent durement leurs enfants car ils croient être encore avec des Algériens.

Ils menacent leurs femmes car «toute la journée, je menace et j'exécute».

Ils ne dorment pas, parce qu'ils entendent les cris et les lamentations de leurs victimes.

De tels faits posent évidemment certains problèmes. Sommes-nous en présence d'hommes torturés par le remords?

17 Dans le Constantinois, un commissaire de police devait se suicider en 1956. 
S'agit-il d'une révolte de la conscience morale?

Les tortures reconnues par ces policiers constituent-elles des exceptions?

L'existence de ces policiers à la limite du pathologique indique-telle le caractère inhabituel, inaccoutumé, somme toute illégal de la torture?

Autrement dit, le policier tortionnaire est-il en contradiction avec les « valeurs » de son groupe et du système qu'il défend?

Après avoir nié l'existence des tortures en Algérie, les Français ont utilisé un double argument.

D'abord, a-t-il été affirmé, il s'agit de cas exceptionnels.

La plus grande démission des intellectuels français est d'avoir toléré ce mensonge. Des sanctions vont être prises, a dit le Gouvernement français, niais nous ne devons pas les rendre publiques. Comme si la torture d'un homme ou le massacre organisé ne relevaient pas tous deux du droit criminel public. La passion de la vérité et de la justice ne peut, sans se contester, accepter pareille supercherie.

[75]

\section{La fuite devant les responsabilités}

Mais les témoignages se faisaient de plus en plus nombreux, les tortures se révélant de moins en moins exceptionnelles, toute responsabilité fut rejetée sur des éléments étrangers servant dans l'armée française. Ce deuxième argument est important. Il montre à la fois le cynisme des autorités françaises et l'impossibilité croissante de ruser, de dissimuler, de mentir. Les Français, depuis un an, ne cessent de répéter que seuls d'anciens S.S. servant dans la Légion sont responsables des tortures. Or, la majorité des déserteurs de l'armée française sont des légionnaires étrangers. C'est parce que les méthodes policières françaises les révoltent que ces Allemands et ces Italiens abandonnent les rangs ennemis et rejoignent les unités de l'A.L.N. C'est par dizaines que nous les interrogeons avant leur rapatriement. Ces an- 
ciens légionnaires sont unanimes: la cruauté et le sadisme des forces françaises sont effroyables.

En tout état de cause il importe de ne pas oublier que l'apparition de soldats tortionnaires remonte à l'hiver 1955. Pendant près d'un an, seuls les policiers ont torturé en Algérie.

On possède aujourd'hui des précisions sur les méthodes employées par les Français. De multiples témoignages ont été publiés et l'importante gamme des techniques, répertoriée. Toutefois, aucun élément n'a été fourni sur la doctrine, la philosophie de la torture. Des renseignements parvenus au F.L.N. éclairent singulièrement cette rationalisation.

\section{Lofrédo et Podevin, théoriciens de la torture.}

Les policiers français Lofrédo (commissaire à Alger) et Podevin (chef de la police judiciaire de Blida) ont précisé, à l'intention de leurs amis et au cours d'exposés techniques à leurs nouveaux collaborateurs, certaines des caractéristiques de leurs méthodes.

1) Plusieurs témoignages et des rapports convergents d'indicateurs désignent un Algérien comme jouant un rôle important dans l'organisation locale du F.L.N. Le patriote est [76] arrêté et conduit dans les locaux de la P.J. On ne lui pose aucune question car, à ce moment de l'enquête, «nous ne connaissons pas la direction que doit prendre l'interrogatoire et le suspect ne doit pas se rendre compte de notre ignorance ». Le meilleur moyen consiste à briser sa résistance en utilisant la méthode dite de «mise en train par l'exemple».

Quelques jeeps quittent la P.J. et ramènent une dizaine d'Algériens ramassés au hasard dans la rue ou, plus fréquemment, dans un douar environnant. Les uns après les autres, en présence du suspect qui, seul, intéresse la police, ces hommes vont être torturés jusqu'à la mort. On estime qu'après 5 ou 6 assassinats, le véritable interrogatoire peut commencer. 
2) La deuxième méthode consiste à torturer d'abord l'intéressé. Plusieurs séances sont nécessaires pour casser son énergie. Aucune question n'est posée au suspect. L'inspecteur Podevin, qui a largement utilisé cette méthode à Blida puis à Alger, avoue qu'il est difficile de ne rien dire lorsque le torturé demande des explications. Aussi faut-il se dépêcher de briser sa résistance.

À la sixième ou septième séance, on se contente de lui dire: on t'écoute.

Ici l'interrogatoire n'est absolument pas orienté. Le suspect, en principe, doit dire tout ce qu'il sait.

Dans les deux cas, le même phénomène est retrouvé: l'interrogatoire est différé.

Dans cette perspective où l'excuse de la fin tend de plus en plus à se détacher des moyens, il est normal que la torture devienne sa propre justification. Et le système colonialiste, pour être logique, doit accepter de revendiquer la torture comme un de ses éléments importants.

\section{Les intellectuels français et la presse française}

M. Martin-Chauffier, dans un rapport prudent où il n'est pas difficile de découvrir une semi-approbation, ne peut échapper à ce dilemme. L'argument de la torture exceptionnelle est repris ici avec une particulière vigueur. Toutefois l'auteur en arrive à reconnaître que « commis à l'étage inférieur, [77] ces crimes se trouvent en quelque sorte couverts par la négligence des pouvoirs supérieurs à en prendre un suffisant souci, et menacent par la quasi-impunité qui les encourage à s'ériger en un véritable système ». La contradiction ne peut guère être niée et à la phrase suivante c'est un I.G.A.M.E., la plus haute autorité française en Algérie, qui approuve, conseille et légitime ces crimes. La prétendue ignorance des pouvoirs supérieurs est manifestement un mensonge et une duplicité. 
M. Martin-Chauffier serait très étonné d'apprendre que son attitude est jugée ici incompréhensible. En réalité, la torture n'est pas un moyen d'obtenir des renseignements. On torture en Algérie par perversion sadique et c'est le seul mot valable du rapport de M. MartinChauffier : "Ce système, dit-il, a pour effet de pervertir ceux qui s'en font les instruments ».

M. G. M. Mattei, qui a participé aux expéditions françaises en Algérie, vient de publier quelques pages dans le numéro de juillet-août des «Temps Modernes». "Je me souviens, écrit-il, que de temps en temps, lorsque le cinéma ambulant du bataillon venait nous projeter un film, et qu'il ne plaisait pas, des soldats et des officiers se levaient et allaient tranquillement passer la fin de la soirée en compagnie des prisonniers... Les cris étaient en partie couverts par la musique du film ».

M. Mattei se révolte contre ces atteintes à la dignité et à l'honneur français. Et naturellement, il termine son témoignage par l'argument désormais classique des démocrates français: «Quelle génération nous prépare-t-on dans ce bouillon de culture qu'est aujourd'hui l'Algérie...? Car «le plus grave», évidemment, «c'est ce qu'étaient devenus, après douze mois d'A.F.N., ces jeunes rappelés avec qui j'ai passé six mois : de véritables mercenaires ».

On ne peut trouver de meilleur exemple de ce que l'on doit somme toute appeler perversion du sens moral. Lorsque les intellectuels français, avec M. Mattei, reprennent en chœur « qu'il y a actuellement en Algérie une vaste entreprise de déshumanisation de la jeunesse française » ou déplorent que les appelés français «y apprennent le fascisme ", il faut savoir que seules les conséquences morales de ces crimes sur l'âme des Français intéressent ces humanistes. La gravité des [78] tortures et des «corvées de bois», l'horreur des viols de fillettes algériennes, sont perçues parce que leur existence menace une certaine idée de l'honneur français.

Il vaut la peine de méditer sur cette attitude. Pareille exclusion de l'Algérien, pareille ignorance de l'homme torturé ou de la famille massacrée, constituent un phénomène entièrement original. Il s'apparente à cette forme de pensée égocentrique, sociocentrique, qui est devenue la caractéristique des Français.

En réalité, il semble que la crainte d'une contamination morale (?) soit totalement vaine. Les policiers malades n'étaient point tourmentés 
par leurs consciences. S'ils maintiennent le rythme professionnel en dehors de leurs bureaux ou de leurs ateliers, en l'occurence les salles de tortures, c'est parce qu'ils sont victimes du surmenage. Ce que réclamaient ces policiers, c'est moins un apaisement moral que la possibilité de reprendre les tortures.

\section{Le système en question}

Le policier qui torture en Algérie n'enfreint aucune loi. Ses actes se situent dans le cadre de l'institution colonialiste. En torturant, il manifeste une réelle fidélité au système. Aussi les soldats français ne peuvent-ils guère faire autrement sans condamner la domination française. Tout Français en Algérie doit se comporter en tortionnaire. Voulant rester en Algérie, il n'y a pour la France d'autre solution que le maintien d'une occupation militaire permanente et d'une puissante structure policière.

Les forces ennemies ne peuvent s'imaginer à quel point il leur est impossible de faire autre chose que d'évacuer le territoire national.

Le peuple algérien ne lutte pas contre les tortures, le viol des fillettes ou les assassinats collectifs. L'histoire de l'occupation française est jalonnée de tels crimes et en Kabylie, récemment encore, on faisait peur aux enfants en les menaçant d' « appeler Bugeaud ».

Le peuple algérien n'ignore pas que la structure colonialiste repose sur la nécessité de torturer, de violer et de massacrer.

[79]

Aussi notre revendication est-elle d'emblée totale et absolue.

Les policiers sadiques qui ont perdu le sommeil et les soldats tortionnaires qui « risquent de se transformer en fascistes » nous posent, à nous Algériens, un problème précis. Comment modifier notre stratégie et intensifier notre combat pour que le territoire national soit, le plus tôt possible, libéré?

Toute autre considération nous est radicalement étrangère. 
[81]

\author{
Pour la révolution africaine \\ Écrits politiques
}

Quatrième partie. Vers la libération de l'Afrique.

Chapitre 3

\title{
À propos d'un plaidoyer ${ }_{18}$
}

\section{Retour à la table des matières}

Par delà les exécutions collectives ou les salles de tortures les démocrates français s'adressent quelquefois au peuple algérien et lui demandent de ne point réunir sous le même mépris et sous la même haine les éléments différents qui représentent le peuple français.

M. Georges Arnaud rappelle, non sans amertume que tout en Algérie, et tout d'abord la condamnation à mort d'une innocente et d'une déséquilibrée intoxiquée par ses bourreaux, se fait au nom du peuple français.

Georges Arnaud, depuis trois ans, le peuple algérien est massacré au nom du peuple français.

Votre plaidoyer pour Djamila Bouhired vous honore mais craignez qu'il ne laisse de côté l'essentiel. L'assassinat de Djamila Bouhired ne pose aucun problème au peuple algérien.

Le rire de Djamila Bouhired à l'annonce de sa condamnation à mort, que personne ne s'y trompe, n'est ni bravade stérile, ni inconscience. 
Ce sourire est bien plutôt la manifestation tranquille d'une certitude intérieure demeurée inébranlable. Le peuple algérien n'a manifesté aucune surprise en apprenant la condamnation à mort de Djamila Bouhired. Car il n'y a pas une famille algérienne [82] qui ne soit blessée, endeuillée, décimée au nom du peuple français.

Le message de Djamila Bouhired s'inscrit dans la tradition des Algériens tombés pour une Algérie indépendante. Les soldats de l'Armée nationale, les hommes et les femmes d'Algérie sont engagés, comme Djamila Bouhired, dans un combat implacable contre la domination étrangère.

Georges Arnaud, il y a eu depuis de multiples Djamila Bouhired, torturées, violées et massacrées sur le territoire algérien.

Il y en aura d'autres et le peuple algérien le sait. Il sait que l'espoir du colonialisme français est d'ébranler la volonté nationale par ces exécutions.

La caractéristique de la majorité des démocrates français est précisément de ne s'alarmer qu'à propos des cas individuels juste bons à arracher une larme ou à provoquer de petites crises de conscience.

On mesure ici la réalité du retard historique de la conscience française. A la lutte pour le respect des libertés individuelles et les droits de l'Homme, si féconde il y a deux siècles, elle n'arrive pas à substituer la lutte pour les droits des peuples. D'où cette crispation sur des cas précis et le vain espoir d'intéresser le peuple français à l'ensemble à partir de situations limites.

La situation-limite ce n'est ni Bouhired, ni Zeddour ni même le stade de Philippeville.

La situation-limite c'est la volonté de douze millions d'hommes. C'est la seule réalité. Et elle n'est pas simplifiable.

Pensez-vous vraiment, Georges Arnaud, rendre service au peuple français en lui parlant de Djamila Bouhired. Quand bien même Djamila Bouhired serait pardonnée (de quoi ?) et graciée, est-ce que la lutte du peuple algérien et la répression faite au nom du peuple français changeraient de forme?

Il est vrai que votre livre a été écrit pour un public français. Il est vrai aussi qu'il existe en France depuis quelque temps des habitudes 
fascistes dont font les frais les écrivains qui se respectent. A cause de tout cela votre initiative est courageuse.

Ce qui est essentiel, voyez-vous Georges Arnaud, c'est de ne pas brouiller les cartes. C'est de ne pas présenter Djamila [83] Bouhired comme une pauvre fille victime de la méchanceté. Djamila Bouhired est une patriote algérienne consciente, organisée au sein du F.L.N.

Elle ne demande ni commisération, ni pitié. La dignité de Djamila Bouhired, son extraordinaire ténacité, son obstination à se tenir debout, à ne pas parler, son souci de sourire devant la mort, constituent les caractéristiques essentielles de l'attitude nationale du peuple algérien.

La mort de Djamila Bouhired, vous avez raison Georges Arnaud, pose un problème au peuple français.

Nous devons toutefois reconnaître que depuis trois ans il ne semble pas que ce peuple ait aperçu la responsabilité effroyable qu'il prenait devant le monde et devant l'histoire en cautionnant, en participant à cette guerre d'Algérie dont on a pu dire qu'elle était la plus grande honte de notre époque.

Maître Jacques Vergès n'a pas pu plaider pour Djamila Bouhired. Vous dites, Georges Arnaud, qu'il s'en est fallu de peu qu'il n'ait été lynché par cette partie du peuple français qui règne à Alger.

Voici donc un nouveau prétexte à révolte: les droits de la défense, la protection de la défense...

Que nous sommes loin de cette guerre qui, il faudra bien le reconnaître un jour, concerne deux peuples.

Quant à Jacques Vergès, originaire de la Réunion, colonie française, il nous suffit de nous souvenir comment plusieurs d'entre nous furent piétinés à Lyon il y a dix ans pour nous sentir de plain-pied avec lui.

Il y a dix ans, des centaines d'ouvriers et d'étudiants algériens manifestant leur solidarité à un parent de Maître Vergès, victime d'un complot colonialiste à la Réunion, étaient matraqués par la police et la gendarmerie françaises.

Y a-t-il si loin de la Réunion à Alger? 


\author{
Pour la révolution africaine \\ Écrits politiques
}

Quatrième partie. Vers la libération de l'Afrique.

\title{
Chapitre 4
}

\section{Les intellectuels et \\ les démocrates français devant la révolution algérienne 19}

\section{I}

\section{Retour à la table des matières}

L'un des premiers devoirs des intellectuels, réunis en l'occurrence sous le terme d'« intelligentzia», et des éléments démocratiques des pays colonialistes, est de soutenir sans réserve la revendication nationale des peuples colonisés. Cette conduite se fonde sur des données théoriques très importantes: défense d'une idée de l'homme, contestée en pays occidental, refus de participer institutionnellement à la dégradation et à la négation de certaines valeurs, communauté d'intérêts entre les classes laborieuses du pays conquérant et de l'ensemble de la population du pays conquis et dominé, enfin souci

19 Cette série de trois articles est parue dans El Moudjahid des ler décembre, 15 décembre, 30 décembre 1957. 
d'imposer à son gouvernement le respect du droit des peuples à disposer d'eux-mêmes.

Ce soutien et cette solidarité se ramènent, avant la période de la lutte armée, à la tenue de quelques meetings annuels et au vote de motions. Quelquefois à la suite d'une répression soudain très féroce, signe avant-coureur d'une répression plus systématisée, plus globale (dans le cas de l'Algérie, les élections de M. Naegelen et le complot de 1950-1951), des campagnes de presse, des déclarations, des mises en gardes, des appels voient le jour.

[86]

Il faut signaler que nulle tentative d'explication n'est amorcée au niveau de l'ensemble de la population du pays colonialiste. Parce qu'elle n'a pas de prise sur le peuple, sur le pays, la gauche démocratique, fermée sur elle-même, se convainc à longueur d'articles ou d'études que Bandoeng a sonné le glas du colonialisme. Or c'est le peuple réel, les paysans et les ouvriers qu'il faut informer. Incapable d'expliquer, de commenter à l'échelle des millions d'ouvriers et de paysans du peuple colonialiste des réalités du drame qui commence, la gauche se trouve acculée au rôle de Cassandre. Elle annonce les cataclysmes mais le manque de préparation de l'opinion publique fait que ces prophéties, inexplicables dans la période pré-insurrectionnelle, seront assimilées à la complicité au moment de l'explosion.

\section{Une inefficacité douloureuse}

Aussi, dans le cas particulier de l'Algérie, après la phase aiguë préinsurrectionnelle (1952-1953), lorsque commence la période (sabotages, attentats) de la phase armée, va-t-on se trouver devant une gauche désemparée et paradoxalement prise au dépourvu.

Les éléments démocratiques et les intellectuels français savent les données du problème. Pour les avoir vues de très près et les avoir étudiées pendant longtemps, ils en connaissent la complexité, la profondeur et la tension. Mais tout le savoir se révèle vain parce qu'incommensurable eu égard aux idées simples qui ont cours dans le peuple. 
Encombrée par ce savoir inutilisable, la gauche bénéficie d'un statut d'augure. Pendant longtemps elle répétera aux gouvernants : « vous étiez prévenus, tout cela arrive par votre faute. »

Dans cette phase effervescente d'alignement des forces et d'organisation de la lutte armée du peuple colonisé, on assiste à une quasi-communication entre le peuple révolté et les éléments démocratiques. C'est que très souvent les intellectuels et les démocrates ont connu personnellement les chefs actuels de la lutte armée. Il s'installe donc entre eux une sorte d'apparente complicité. Mais, très rapidement, cette pseudo-solidarité active sera balayée par les événements. En effet, au cours de la deuxième période, caractérisée par des engagements, des embuscades et des attentats, la culpabilité si généreusement [87] rejetée sur les responsables officiels tend à se déplacer. La répression s'approfondit, s'organise, se nuance. Les chambres de torture apparaissent. Sur tout le territoire national algérien des dizaines et des centaines de patriotes sont assassinés.

Le peuple concret, les hommes et les femmes, les enfants et les vieillards du pays colonisé s'aperçoivent sans effort qu'exister au sens biologique du mot et exister en tant que peuple souverain coincident. La seule issue possible, l'unique voie de salut pour ce peuple est de répondre aussi énergiquement qu'il le peut à l'entreprise de génocide menée contre lui.

La réponse se fait de plus en plus absolue.

\section{Le nationalisme et la « barbarie »}

C'est ici que se situe un double phénomène. D'abord une propagande ultra-chauvine, nationaliste, patriotique, mobilisant les éléments racistes implicites de la conscience collective du peuple colonialiste, apporte un nouvel élément. Dès lors il devient évident qu'il n'est plus possible de soutenir le colonisé sans du même coup s'opposer à la voie nationale. La lutte contre le colonialisme devient lutte contre la nation. La guerre de reconquête est assumée par l'ensemble du pays colonialiste et les arguments anti-colonialistes perdent de leur efficacité, deviennent des théories abstraites et, à l'extrême, disparaissent de la littérature démocratique. 
Dans le cas de l'Algérie, c'est à partir de mars 1956, avec le rappel du contingent, que la nation française a pris en main la guerre de reconquête coloniale. Les manifestations de rappelés furent à ce moment les derniers symptômes d'une guerre jugée impopulaire sur le plan doctrinal.

Depuis 1956, la guerre d'Algérie est acceptée par la nation. La France veut la guerre, diront explicitement M. Guy Mollet et M. Bourgès-Maunoury; et le peuple parisien, le 14 juillet 1957, exprimera aux parachutistes tortionnaires de Massu la grande reconnaissance de la patrie. Les libéraux abandonnent la lutte à cette phase. L'accusation de trahison qui guette les adversaires de la guerre d'Algérie devient une arme redoutable entre les mains du gouvernement français. Ainsi a-t-on pu voir au début de l'année 1957 un grand nombre de démocrates se taire ou être fauchés par la vague revancharde et [88] élaborer un patriotisme élémentaire mal structuré, hanté par le racisme, violent, totalitaire, somme toute fasciste.

Le gouvernement français trouvera son deuxième argument dans ce qu'on appelle le terrorisme. Les bombes à Alger seront exploitées par le service de propagande. Enfants blessés, innocents, qui ne s'appellent pas Borgeaud ou qui ne répondent pas à la classique définition du «féroce colonialiste » posent aux démocrates français des problèmes inattendus. La gauche est ébranlée: Sakamody renforcera ce recul. Dix civils français sont tués dans une embuscade et toute la gauche française, dans un unanime sursaut, de s'écrier: on ne vous suit plus. La propagande s'orchestre, s'insinue dans les esprits et démantèle les convictions déjà largement fissurées. Le concept de barbarie apparaît et il est décidé que la France en Algérie combat la barbarie.

Une grande partie des intellectuels, la presque totalité de cette gauche démocratique, s'effondre et pose au peuple algérien ses conditions: condamnez Sakamody et les bombes et nous vous conservons notre amical appui.

À l'aube de la quatrième année de la guerre de libération nationale, face à la nation française et face aux bombes de la rue Michelet, la gauche française se fait de plus en plus absente.

Quelques-uns se sont réfugiés dans le silence, d'autres ont choisi certains thèmes qui, épisodiquement, réapparaissent. La guerre l'Algérie doit cesser car elle coûte cher (la guerre d'Algérie redevient 
impopulaire parce que, simplement, elle coûte 1.200 milliards de francs), isole la France ou permet son remplacement par les AngloSaxons ou par les Russes ou par Nasser, etc...

En France, on sait de moins en moins pourquoi la guerre d'Algérie doit prendre fin. On oublie de plus en plus que la France, en Algérie, piétine la souveraineté populaire, bafoue le droit des peuples à disposer d'eux-mêmes, assassine des milliers d'hommes et de femmes.

La guerre d'Algérie tend à devenir, en France, au sein de la gauche, une maladie du système français comme l'instabilité ministérielle; les guerres coloniales un tic de la France, une partie du panorama national, un détail coutumier.

[89]

\section{II}

Depuis 1956, les intellectuels et les démocrates français s'adressent périodiquement au F.L.N. La plupart du temps, il s'agit soit de conseils politiques, soit de critiques concernant telle physionomie de la guerre de libération. Cette attitude de l'intelligentzia française ne doit pas être interprétée comme la conséquence d'une solidarité, interne avec le peuple algérien. Ces conseils et ces critiques s'expliquent par le désir difficilement réprimé de guider, d'orienter jusqu'au mouvement de libération de l'opprimé.

Ainsi se comprend l'oscillation constante des démocrates français entre une hostilité manifeste ou latente et l'aspiration totalement déréelle à militer "activement jusqu'au bout ». Une telle confusion indique le manque de préparation aux problèmes concrets et la noninsertion des démocrates français sur le plan de la vie politique intérieure française.

Le long de cette ligne d'oscillation, les démocrates français, en dehors de la lutte ou manifestant la volonté de l'observer de l'intérieur, voire d'y participer en qualité de censeurs, de conseillers, incapables ou refusant de choisir un terrain précis ou se battre à l'intérieur du dispositif français, menacent et pratiquent le chantage. 
La pseudo-justification de cette attitude est que, pour avoir une influence sur l'opinion française, il faut condamner certains faits, rejeter les excroissances inattendues, garder ses distances devant les « excès». Dans ces moments de crise, d'affrontement, il est demandé au F.L.N. d'orienter la violence, et de la faire sélective.

\section{Le mythe de l'Algérie française}

À ce niveau, la réflexion nous permet de découvrir une particularité importante du fait colonial algérien. Au sein d'une nation, il est classique et banal d'identifier deux forces antagonistes : la classe ouvrière et le capitalisme bourgeois. En pays colonial cette distinction se révèle totalement inadéquate. Ce qui définit la situation coloniale c'est bien plutôt le caractère indifférencié que présente la domination étrangère. La situation coloniale c'est d'abord une conquête militaire continuée et renforcée par une administration civile [90] et policière. En Algérie, comme dans toute colonie, l'oppresseur étranger s'oppose à l'autochtone comme limite de sa dignité, et se définit comme contestation irréductible de l'existence nationale.

Le statut de l'étranger, du conquérant du Français en Algérie, est un statut d'oppresseur. Le Français en Algérie ne peut être neutre ou innocent. Tout Français en Algérie opprime, méprise, domine. La gauche française, qui ne peut rester indifférente et imperméable à ses propres fantasmes, adopte en Algérie, dans la période antérieure à la guerre de libération, des positions paradoxales.

\section{Qu'est-ce que le colonialisme?}

Les démocrates français, en décidant de nommer colonialisme ce qui n'a jamais cessé d'être conquête et occupation militaire, ont délibérément simplifié les faits. Le terme de colonialisme créé par l'oppresseur est trop affectif, trop émotionnel. C'est situer un problème national sur un plan psychologique. C'est ainsi que, dans l'esprit de ces démocrates, le contraire de colonialisme n'est point reconnaissance du droit des peuples à disposer d'eux-mêmes, mais nécessité à 
l'échelon individuel de comportements moins racistes, plus ouverts, plus libéraux.

Le colonialisme n'est pas un type de relations individuelles mais la conquête d'un territoire national et l'oppression d'un peuple: c'est tout. Ça n'est pas une certaine conduite humaine ou une modalité de rapports entre individus. Tout Français en Algérie actuellement est un soldat ennemi. Tant que l'Algérie ne sera pas indépendante, il faut accepter cette conséquence logique. M. Lacoste l'a compris qui a « mobilisé en surface » les Français et les Françaises résidant en Algérie.

Au terme de cette analyse, on s'aperçoit que, loin de reprocher au Front de Libération Nationale certaines de ses actions urbaines, on devrait au contraire apprécier les efforts qu'il impose au peuple.

C'est faute d'avoir compris que le colonialisme n'est qu'une domination militaire, que les démocrates français se trouvent aujourd'hui à la limite du paradoxe.

Victimes du mythe de l'Algérie française, les partis de [91] gauche créent sur le territoire algérien des sections algériennes des partis politiques français. Les mots d'ordre, les programmes, les modes de lutte sont identiques à ceux de la «métropole». Une position doctrinale, incontestée jusqu'à ces derniers temps, a justifié cette attitude. En pays colonial, disait-on, il y a entre le peuple colonisé et la classe ouvrière du pays colonialiste une communauté d'intérêts. L'histoire des guerres de libération menées par les peuples colonisés est l'histoire de la non-vérification de cette thèse.

\section{Le colonialisme n'est pas M. Borgeaud}

Le peuple algérien se montre réfractaire à l'imagerie simpliste qui veut que le colonialiste soit un type particulier d'homme facilement reconnaissable. C'est ainsi qu'il a été avancé que tous les Français en Algérie ne sont pas des colonialistes, ou qu'il a été décrit des degrés dans le colonialisme. Or ni M. Borgeaud ni M. de Sérigny ne caractérisent totalement le colonialisme français en Algérie. Le colonialisme français, l'oppression française en Algérie, forment un ensemble cohérent qui ne requiert pas forcément l'existence de $M$. Borgeaud. La domination française, c'est la totalité des forces qui s'opposent à 
l'existence de la nation algérienne, et pour l'Algérien, concrètement, M. Blachette n'est pas plus « colonialiste » qu'un agent de police, un garde-champêtre ou un instituteur.

L'Algérien ressent globalement le colonialisme français, non par simplisme ou xénophobie mais parce que, réellement, tout Français en Algérie entretient avec l'Algérien des rapports basés sur la force. L'évocation de cas particuliers de Français anormalement gentils avec les Algériens ne modifie pas la nature des relations entre un groupe étranger qui s'est accaparé les attributs de la souveraineté nationale et le peuple qui se trouve privé de l'exercice du pouvoir. Aucune relation personnelle ne peut contredire cette donnée fondamentale : que la nation française par l'intermédiaire de ses ressortissants s'oppose à l'existence de la nation algérienne.

Dans les colonies d'encadrement, le peuple colonialiste est représenté par les soldats, les policiers et les techniciens. Le peuple colonialiste peut dans ces conditions se réfugier dans l'ignorance des faits et se déclarer innocent de la colonisation. [92] Dans les colonies de peuplement cette fuite devant soi-même devient impossible. Parce que, selon la formule célèbre d'un chef d'Etat français, « il n'existe pas un Français qui n'ait un cousin en Algérie » toute la nation française s'est trouvée engagée dans le crime contre un peuple et se trouve aujourd'hui complice des assassinats et des tortures qui caractérisent la guerre d'Algérie.

Le démocrate français authentique doit être non contre $M$. Borgeaud ou contre M. Blachette, il doit éviter de choisir arbitrairement quelques boucs émissaires qui ne peuvent exprimer les 130 années d'oppression colonialiste. Le démocrate français doit juger et condamner l'ensemble de la colonisation ramené à sa catégorie d'oppression militaire et policière. Il faut se convaincre que tout Français en Algérie réagit comme $M$. Borgeaud. C'est qu'il n'existe pas en Algérie de Français qui ne soit justifié dans son existence même par cette domination.

Parce qu'il ne peut adopter cette attitude par absence de courage on défaut d'analyse, le démocrate français se réfère constamment à des abstractions: le colonialisme en général se meurt, le colonialisme est inhumain, la France doit être fidèle à son histoire, oubliant ainsi 
singulièrement que le colonialisme constitue une part importante de l'histoire française.

Le colonialisme est l'organisation de la domination d'une nation après la conquête militaire. La guerre de libération n'est pas quête de réformes mais effort grandiose d'un peuple, qu'on avait momifié pour retrouver son génie, pour reprendre en main son histoire, et s'installer souverain.

Des Français, dans le cadre de I'O.T.A.N., refusent de servir sous les ordres du général allemand Speidel mais acceptent de se battre contre le peuple algérien. Or, en toute rigueur, la fidélité à l'esprit de la résistance française voudrait que les Français qui répugnent à servir sous Speidel, logiques avec eux-mêmes, refusent de combattre sous Massu ou Salan.

\section{III}

De toute évidence, les gouvernants français ont raison lorsqu'ils prétendent que le problème algérien ébranle les bases [93] mêmes de la République. Depuis quelques années, le mythe de l'Algérie française a été soumis à une rude épreuve, et dans la conscience française, il s'est installé une dose d'incertitude quant à la vérité de cette thèse.

À l'échelon international on a pu enregistrer des répercussions de cette destruction. Toutefois, de tels progrès n'ont pas résolu totalement le problème de la mystification engendrée par des dizaines d'années d'enseignement mensonger et de falsification historique systématisée.

\section{Le prix de la mystification}

Quand on analyse de près les rapports colonialistes qui ont existé entre l'Algérie et la France, on s'aperçoit que le territoire algérien, par les caractères mêmes de sa conquête, a toujours représenté pour la France un prolongement plus ou moins réel. A aucun moment la Fran- 
ce n'a indiqué dans les termes identiques son droit de propriété sur l'Afrique Noire ou sur une autre parcelle de «l'Empire français». On a pu décréter l'Afrique Noire terre française, mais jamais il ne fut décidé que l'Afrique Noire était la France.

Le droit de la France en Afrique se ramenait davantage à un droit de propriété, tandis qu'en Algérie, dès le début, des rapports d'identité étaient affirmés. Nous avons vu que les démocrates français, à de rares exceptions, ont adapté leur attitude à cette optique. Les partis politiques français n'ont pas caché la nécessité dans laquelle ils se trouvaient d'obéir à cette mystification. M. Laurent Casanova, dans un discours aux étudiants communistes prononcé le 17 mars 1957 à Paris, répondant aux critiques que lui adressait la jeunesse communiste sur l'attitude du Parti Communiste Français en face du problème algérien, se justifiait en lui demandant de tenir compte de «l'attitude spontanée des masses populaires française sur la question ».

Parce que pendant 130 ans la conscience nationale française s'est élaborée à partir d'un principe de base simple: l'Algérie est la France, on se heurte aujourd'hui, au moment où une grande partie du peuple français se rend compte rationnellement que son intérêt va dans le sens de la fin de la guerre et de la reconnaissance d'un Etat algérien indépendant, à des réactions instinctives, passionnelles, antihistoriques.

Jamais le principe qui veut que personne n'asservit impunément [94] ne fut aussi totalement vrai. Après avoir domestiqué pendant plus d'un siècle le peuple algérien, la France se trouve prisonnière de sa conquête et incapable de s'en détacher, de définir de nouveaux rapports, de prendre de nouvelles orientations.

\section{Un marchandage odieux}

La grande erreur, d'ailleurs serait de croire le problème épuisé par ces considérations psychologiques. Les confrontations avec les représentants de la gauche française font apparaître des préoccupations beaucoup plus complexes. C'est ainsi que, sur le point précis de l'avenir de l'Algérie indépendante, nous nous trouvons devant deux exigences contradictoires qui se recoupent d'ailleurs, à un échelon plus élevé, 
avec la conception manichéiste du bien et du mal qui depuis quelques années partage le monde.

La gauche non-communiste nous assure de son appui, nous promet ses interventions, mais nous demande de lui garantir que jamais l'Algérie ne sombrera dans le bloc communiste ou dans le bloc dit neutraliste. L'anti-colonialisme de ces démocrates n'est donc pas sans réserves et inconditionnel, mais suppose un choix politique précis. Certes, les arguments ne leur manquent pas. Troquer le colonialisme français contre le « colonialisme » rouge ou nassérien leur paraît une opération négative, car, affirment-ils, à l'heure contemporaine des grands ensembles, un alignement est obligatoire et leurs conseils ne sont pas voilés: il faut choisir le bloc occidental.

Cette gauche non-communiste est généralement réticente quand nous lui expliquons que, pour le moment, il s'agit pour le peuple algérien d'abord de se libérer du joug colonialiste français. Refusant de se maintenir sur le plan strict de la décolonisation et de la libération nationale, la gauche française non-communiste nous adjure de jumeler les deux efforts : refus du colonialisme français et du communisme soviéto-neutraliste,

Le même problème, selon un dynamisme inverse, se pose avec la gauche française communiste. Le Parti Communiste Français, dit-elle, ne peut appuyer que certains mouvements de libération nationale, car quel intérêt représenterait pour nous, communistes français, l'irruption de l'impérialisme américain [95] en Algérie. Ici encore des garanties nous sont demandées, on veut nous arracher des promesses. On réclame des assurances.

On comprend que de telles difficultés gênent l'action anticolonialiste de la gauche française. C'est que l'Algérie non encore indépendante fait déjà l'objet de luttes d'influence à l'échelon international. Pour qui va donc se libérer l'Algérie? Le peuple algérien depuis trois ans ne cesse de répéter qu'il se propose de se libérer pour son propre compte, que ce qui est important pour lui c'est d'abord de reconquérir sa souveraineté, d'asseoir son autorité, de réaliser son humanisation, sa liberté économique et politique: mais il ne semble pas que ces évidences soient acceptées.

Le peuple algérien souffre de façon terrifiante sa naissance à l'indépendance et déjà on lui monnaie, avec une agressivité inaccoutu- 
mée, la moindre parcelle d'appui. C'est ainsi qu'il n'est pas rare d'entendre certains Français démocrates nous dire : aidez-nous à vous aider. Ce qui signifie clairement: dites-nous un peu où vous comptez aller après.

Cette mise en demeure, qui se situe toujours à l'échelon individuel entre Français et Algériens, représente certainement l'un des aspects les plus douloureux de la lutte pour l'indépendance. Certains démocrates français sont quelquefois heurtés par la sincérité du combattant algérien. C'est que le caractère total de la guerre que nous menons se répercute sur la manière non moins radicale que nous avons de vivre les échanges individuels. Et il nous faut avouer que cela nous est insupportable de voir certains Français que nous avions crus être nos amis se comporter avec nous comme des marchands et pratiquer cette sorte de chantage odieux à la solidarité assortie de restrictions fondamentales sur nos objectifs.

\section{Un désaccord fondamental}

Si l'on compare l'attitude de la gauche française à l'égard des buts de notre lutte, on s'aperçoit qu'aucune fraction n'admet l'éventualité d'une libération nationale réelle.

La gauche non-communiste concède que le statut colonial [96] doit disparaître. Mais, entre la liquidation du régime colonial - ramené en l'occurence à un régime préférentiel, avec lutte de castes à l'intérieur d'un ensemble - et la reconnaissance d'une nation algérienne, indépendante de la France, cette gauche a interposé une multitude d'étapes, de sous-étapes, de solutions originales, de compromis.

Il est clair que pour cette partie de la gauche la fin de la guerre d'Algérie doit amener une sorte de fédéralisme interne et d'Union Française rénovée. Notre désaccord avec cette opinion française n'est donc ni d'ordre psychologique ni d'ordre tactique comme certains le prétendent. Les radicaux de gauche, les socialistes minoritaires et les M.R.P. de gauche n'ont pas accepté l'idée d'une indépendance algérienne. Aussi les positions du genre: nous sommes d'accord sur le fond mais pas sur les méthodes sont-elles radicalement fausses. 
La gauche communiste, de son côté, tout en proclamant la nécessaire évolution des pays coloniaux vers l'indépendance, exige le maintien des liens particuliers avec la France. De telles positions manifestent clairement que mêmes les partis dits extrémistes considèrent que la France a des droits en Algérie et que l'allègement de la domination ne doit pas obligatoirement s'accompagner de la disparition de tout lien. Cette disposition d'esprit est présentée sous les formes d'un paternalisme technocratique, d'un chantage à la régression.

Sans liens avec la France, est-il auguré, que ferez-vous?

Il vous faut des techniciens, des devises, des machines...

Il n'est pas jusqu'au tableau catastrophique d'une Algérie mangée par le désert, infestée de marécages et ravagée par les maladies qui ne soit mobilisé pour nous faire réfléchir.

Les colonialistes, dans leur propagande, disent au peuple français: la France ne peut pas vivre sans l'Algérie.

Les anti-colonialistes français disent aux Algériens: l'Algérie ne peut pas vivre sans la France.

Les démocrates français n'aperçoivent pas toujours le caractère colonialiste, où, pour employer un concept nouveau, néocolonialiste de leur attitude.

L'exigence de liens particuliers avec la France répond au désir de maintenir intactes des structures coloniales. Il s'agit ici d'une sorte de terrorisme du nécessaire à partir duquel il est décidé que rien de valable en Algérie ne saurait être conçu ou réalisé en dehors de la France. En fait, la réclamation [97] de liens particuliers avec la France se ramène à la volonté de maintenir éternellement l'Algérie à un stade d'Etat mineur et protégé. Mais c'est aussi garantir certaines formes d'exploitation du peuple algérien. C'est incontestablement faire la preuve d'une incompréhension grave des perspectives révolutionnaires de la lutte nationale. 


\section{Est-il trop tard?}

Il faut que les démocrates français dépassent les contradictions qui stérilisent leurs positions s'ils veulent effectuer une authentique démocratisation avec les colonialistes. C'est dans la mesure où l'opinion démocratique française sera sans réticences que son action pourra être efficace et décisive.

Parce que la gauche obéit inconsciemment au mythe de l'Algérie française, son action se contente de viser une Algérie où règnerait davantage de justice et de liberté, ou, à l'extrême, une Algérie gouvernée moins directement par la France. Le chauvinisme passionnel de l'opinion française sur la question algérienne fait pression sur cette gauche, lui inspire une prudence excessive, ébranle ses principes et la place dans une situation paradoxale et rapidement stérile.

Le peuple algérien estime que la gauche française n'a pas fait tout ce qu'il fallait dans le cadre de la guerre d'Algérie. Il ne s'agit pas pour nous d'accuser les démocrates français, mais d'attirer leur attention sur certaines attitudes qui nous paraissent opposées aux principes de l'anti-colonialisme.

Il n'est peut-être pas inutile de rappeler l'attitude de l'Internationale Socialiste sur cette question. Personne n'ignore qu'en 1956 la délégation française conduite par M. Pineau y fut condamnée et que M. Bevan en 1957, lors du congrès socialiste de Toulouse, exprima publiquement sa déception et sa colère devant le racisme et le colonialisme de la S.F.I.O.

Depuis 1954, le peuple algérien lutte pour l'indépendance nationale. Il s'agit d'un territoire conquis depuis plus d'un siècle qui exprime sa volonté de se constituer en nation souveraine. La gauche française doit soutenir sans réserve cet effort. Ni la présence d'une minorité européenne, ni Sakamody, ne peuvent et ne doivent entamer la détermination [98] d'une gauche authentique. Nous avons vu que la propagande de M. Lacoste ne cesse d'affirmer que la France, en Algérie combat la barbarie. La gauche doit se montrer imperméable à cette campagne et exiger la fin de la guerre et la reconnaissance de l'indépendance de l'Algérie. 
Il est arrivé, avons-nous vu, que certains démocrates utilisent le raisonnement suivant : si vous voulez que notre aide continue, condamnez tels ou tels actes. Ainsi, la lutte d'un peuple pour son indépendance doit être diaphane si elle veut bénéficier de l'appui des démocrates.

Paradoxalement se retrouve ici l'attitude de M. Guy Mollet qui, pour continuer sa guerre, désigne une commission de sauvegarde avec la mission de signaler des "excès», isolant spectaculairement les mauvais soldats de la bonne et juste et féconde armée française.

\section{Les tâches de la gauche française}

Le F.L.N. s'adresse à l'ensemble de la gauche française et lui demande dans cette quatrième année de s'engager concrètement dans le combat pour la paix en Algérie.

$\grave{A}$ aucun moment il ne saurait être question pour les démocrates français de rejoindre nos rangs ou de trahir leur pays. Sans renier sa nation, la gauche française doit lutter pour que le gouvernement de son pays respecte les valeurs qui s'appellent : droit des peuples à disposer d'eux-mêmes, reconnaissance de la volonté nationale, liquidation du colonialisme, rapports réciproques et enrichissants entre des peuples libres.

Le F.L.N. s'adresse à la gauche française, aux démocrates français et leur demande d'encourager toute grève entreprise par le peuple français contre l'élévation du coût de la vie, les nouveaux impôts, la restriction des libertés démocratiques en France, conséquences directes de la guerre d'Algérie.

Le F.L.N. demande à la gauche française de renforcer son action d'information et de continuer à expliquer aux masses françaises les caractéristiques de la lutte du peuple algérien, les principes qui l'animent, les objectifs de la Révolution.

[99]

Le F.L.N. salue les Français qui ont eu le courage de refuser de prendre les armes contre le peuple algérien et qui sont maintenant en prison. 
Ces exemples doivent se multiplier afin qu'il soit clair pour tout le monde et d'abord pour le gouvernement français, que le peuple français refuse cette guerre qui est faite en son nom contre le droit des peuples, pour le maintien de l'oppression, contre le règne de la liberté. 


\author{
Pour la révolution africaine \\ Écrits politiques
}

Quatrième partie. Vers la libération de l'Afrique.

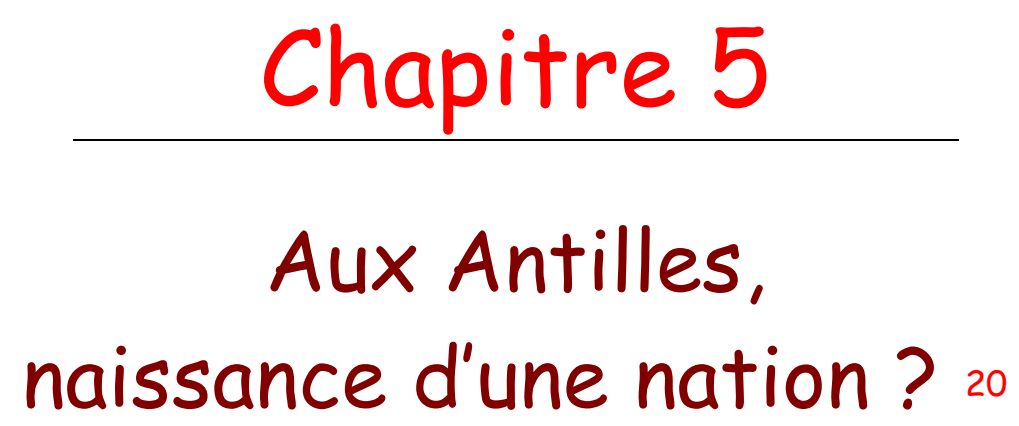

Retour à la table des matières

Le 3 janvier 1958, les «Antilles Britanniques» ont disparu pour faire place à une "Fédération des Indes occidentales» destinées à devenir - comme le Ghana par exemple - un Dominion au sein du Commonwealth.

Une colonie vient donc d'obtenir son autonomie interne, avec promesse d'indépendance, dans l'archipel des Antilles.

Que signifie cet événement pour les peuples d'une des régions du globe les plus anciennement marquées par le colonialisme?

20 El Moudjahid, № 16 du 15 janvier 1958. 


\section{Le sucre roi.}

L'archipel des « Caraïbes», comme on nomme aussi les Antilles (du nom de leurs premiers habitants, les Indiens Caraïbes, tous disparus, premières victimes de l'exploitation blanche dans cette région), est constitué par une poussière d'îles, les unes grandes, les autres minuscules, qui s'alignent entre l'Amérique du Nord et l'Amérique du Sud: elles commandent ainsi le passage vers le canal de Panama, noud de communications essentiel pour l'Amérique.

Toute leur histoire a été marquée par leur situation sous un climat tropical et par la richesse de leur sol qui les rendent particulièrement aptes à la production de la canne à sucre.

Quand les Européens découvrirent l'Amérique, le sucre [102] de betterave n'était pas encore connu: la possession de ces terres à sucre devenait une source de richesses et chaque puissant voulut avoir «son » Antille. Espagnols, Anglais, Français, Hollandais organisèrent, dans leurs domaines respectifs, la production et l'exploitation du sucre de canne, au profit exclusif de la « métropole».

Un problème se posait, celui de la main-d'œuvre ; les Indiens Caraïbes ne résistèrent pas au travail extrêmement dur qui était exigé sur les plantations. Et bientôt, la «traite des Noirs » fut le moyen employé pour les remplacer: des cargaisons entières d'esclaves « importés » d'Afrique dans des conditions épouvantables se déversaient sur les Antilles.

Pendant des siècles, la main-d'œuvre noire fut ainsi enlevée, vendue, achetée, parquée et obligée au travail comme un véritable bétail, travail dont les Etats européens tiraient tout le bénéfice par l'intermédiaire des gros propriétaires, des planteurs blancs, des marchands, des importateurs des ports d'Europe.

Aussi, face à la puissance extraordinaire des planteurs blancs, l'abolition de l'esclavage au XIX siècle se révéla-t-elle inefficace à provoquer l'amélioration réelle de la situation des travailleurs noirs. Ceux-ci durent rester ouvriers agricoles sur les plantations et, encore aujourd'hui, leurs misérables cases voisinent la luxueuse maison du planteur. 
«L'abolition de l'esclavage a laissé le nouvel affranchi aussi dépendant et à la merci du sucre-Roi qu'il l'avait été comme esclave » (Eric Williams).

La « royauté du sucre», forme antillaise du colonialisme, a fait de ces îles, autrefois florissantes, une des régions « sous-développées » du monde.

Culture exclusive d'un produit destiné à l'exportation au lieu de cultures vivrières: absence d'industrialisation: vie chère (car tout doit être importé de la « métropole » située à $7.000 \mathrm{kms}$, ou, au moins, d'Amérique): concentration des terres entre les mains de quelques très gros propriétaires tout puissants sur leurs ouvriers et sur les administrations: misère des paysans sans terre, réduits au chômage, de plus en plus nombreux à cause d'une très forte poussée démographique : essais d'émigration à l'étranger (Porto-Ricains aux U.S.A., Jamaïcains en Angleterre) ou regroupement dans les bidon [103] villes des faubourgs ; analphabétisme, malnutrition, mauvaise santé.

Misère de tous les colonisés, terriblement aggravée par un racisme odieux, séquelle peut-être la plus cruelle de l'esclavage, qui opposa entre eux blancs, noirs et aussi mulâtres. Misère contre laquelle la lutte est spécialement difficile du fait des particularismes créés par quatre siècles de colonisation.

\section{Des colonies juxtaposées}

Car ces îles qui ont en gros le même climat, la même population, les mêmes problèmes économiques et sociaux ont été modelées par des maîtres différents : les Jamaïcains parlent anglais, ou un patois à base d'anglais, lisent des livres anglais, consomment des produits anglais, vont (quand ils peuvent) dans des universités ou des usines anglaises et luttent contre le pouvoir anglais.

Les Porto-Ricains, en majorité d'origine blanche, sont très attachés à la langue de leur ancienne métropole, l'Espagne, même quand ils émigrent en Amérique du Nord. 
Les Antillais de la Martinique et de la Guadeloupe «Département d'Outre-mer » subissent depuis des siècles la politique d'assimilation chère à la France, tendant à les dépouiller systématiquement de leur personnalité, au point que certains d'entre-eux se retrouvent fonctionnaires de la France dans ses autres colonies.

Si Aimé Césaire a pu parler « d'une manière de ghetto insulaire » entre les différentes îles, c'est dire que la solidarité antillaise, inscrite dans les faits et ressentie par les Antillais les plus conscients est loin encore de se traduire dans la vie quotidienne et même dans la lutte d'émancipation: chacune devant d'abord adapter son effort à l'ennemi particulier qu'elle doit vaincre.

Dans toutes les Antilles, le mouvement de libération économique et politique du XIXe Siècle s'est greffé sur une renaissance culturelle à formes multiples: prise de conscience de l'histoire antillaise, réhabilitation des traditions populaires, redécouverte des cultes africains comme forme de résistance à l'oppression occidentale et chrétienne (au «christianisme [104] forcé»), acceptation du passé d'esclavage, fierté d'appartenir à la race noire.

Cette renaissance se manifeste actuellement, avec beaucoup de vigueur, sur le plan intellectuel, à Haïti, aux Antilles françaises et aux Antilles britanniques, où, précisément, un langage commun « le créole » (mélange de français, d'anglais, d'espagnol et de dialectes africains) constitue un lien et un meilleur moyen d'expression de la conscience antillaise.

Quant aux revendications, elles sont d'autant plus énergiques que l'oppression coloniale et l'oppression raciale aggravent l'oppression sociale dans la plupart des îles.

\section{La Fédération des Indes occidentales, futur dominion}

En Jamaïque: les travailleurs de l'industrie sucrière s'organisent à partir de 1920 avec pour leader Bustamante. Une révolte, en 1938, est réprimée par l'armée. À cette époque apparaît un parti politique, le People's National Party (P.N.P.) sous l'impulsion d'un avocat Norman 
Manley. Le P.N.P. est le parti politique d'union nationale le plus puissant : tandis que Bustamante, démagogue, devient dictatorial dans son syndicat (dont il est «président à vie»!) une nouvelle centrale se crée, apparentée au P.N.P.

En 1954, le Parti de Manley est majoritaire dans le pays et à l'Assemblée: une purge, au sein du parti, décapite l'aile gauche qui comptait quelques marxistes et surtout des syndicalistes.

Dès lors, Norman Manley, chef du gouvernement de la Jamaïque, évolue vers le réformisme; il suscite une troisième centrale syndicale et envisage des transformations économiques superficielles avec l'aide, à l'extérieur, des U.S.A. et, à l'intérieur, des planteurs « nationaux». Mais le P.N.P. demeure solide et majoritaire. Manley est un des hommes politiques des Caraïbes qui « pense antillais». Pour lui, une nation antillaise est née, la fédération anglaise n'est qu'une étape. Le statut de Dominion sera la seconde étape, permettant d'envisager une confédération trilingue de toutes les Antilles. Manley reste majoritaire en Jamaïque parce qu'il exprime la conscience nationale antillaise: l'idée d'une nation antillaise fait de grands progrès dans la conscience populaire.

[105]

À la Trinité: la présence de gisements de pétrole a suscité une industrialisation unique aux Antilles.

En 1919, premières grèves de dockers et début d'un grand mouvement de revendication politique, animé par un colon blanc: le « capitaine »Cipriani, qui deviendra le maire de Port Of Spain, la capitale.

En février 1935, nouveaux troubles. En 1937: une marche de la faim sur Port Of Spain se transforme en un véritable soulèvement, mâté par l'armé anglaise. Mais un syndicat est organisé sous l'impulsion d'un actif leader: Butler et, aujourd'hui la «Oil Workers Trade Union » (Syndicat des travailleurs du pétrole) tient une place très importance dans l'organisation économique de liîle.

Depuis 1955, Eric Williams a organisé sur le modèle du P.N.P. de Jamaïque, un «People's National Mouvement » (P.N.M.) qui, en septembre 1956, a obtenu la majorité absolue aux élections : malgré cela, Eric Williams a été expulsé, le P.N.M. n'est pas au pouvoir ; sa première revendication est l'autonomie interne avec suffrage universel. 
À la Barbade: L'exemple du soulèvement de 1937 à la Trinité a été contagieux: Clément Payne organise les premiers syndicats. En 1945, nouveaux troubles, incendie d'une récolte de cannes à sucre. Deux mouvements existent depuis la guerre, un syndicat très puissant des «Travailleurs des plantations sucrières » et un parti politique socialisant, actuellement au pouvoir grâce à l'abolition du double collège depuis 1950 (le premier collège était réservé aux blancs). Le suffrage universel a porté à la présidence Grantley H. Adams, avocat et leader syndical, mais l'autonomie relative de lîle est bridée par les pouvoirs politiques que conserve le gouverneur anglais et par la domination économique qu'exercent les planteurs blancs.

Aujourd'hui, Jamaïque, Trinité, Barbade et les autres petites îles, chacune avec son statut, sont fédérées. L'Angleterre, sous la pression des forces d'émancipation locales et par crainte d'une contamination marxiste venue de Guyane anglaise (où le Docteur Jagan est président depuis 1957), a reconnu l'existence d'une nation antillaise. La voie est ouverte vers l'indépendance et la confédération reste la perspective du travailliste très modéré Manley comme du jeune Eric Williams.

Le 25 mars prochain, des élections au suffrage universel [106] pour le Parlement Fédéral viendront traduire la nouvelle réalité.

\section{Le reste de l'archipel}

Haïti: Haïti résulte de la séparation de l'ancienne colonie française de St Dominique en deux parties: la République Dominicaine de l'Est, où se regroupent les blancs, Haiiti à l'Ouest, avec les gens de couleur.

Les Antilles Françaises « Départements d'Outre-Mer» : L'évolution politique accélérée de la Martinique et de la Guadeloupe date de 1944.

Elles ont fait confiance à la « France de la Libération» pour lutter contre la puissance politico-économique de la « plantocratie sucrière ». La population, qui votait socialiste ou communiste, avait pour première revendication l'égalité sociale; les leaders ont donc joué le jeu de la « départementalisation ».

Les planteurs sont toujours puissants, les ouvriers toujours mal payés, la législation sociale et les allocations familiales sont bien moins 
avantageuses qu'en France: la vie est très chère, à cause de l'union douanière qui oblige à tout importer de France; le pays n'est pas industrialisé, ni la jeunesse scolarisée complètement. Le truquage électoral d'une part, l'inefficacité de six députés antillais, perdus à l'Assemblée Nationale française d'autre part, rendent illusoires les droits politiques attachés, en principe, au titre de citoyen français.

« Nous sommes devenus des départements français et pourtant nous restons plongés dans une effroyable misère. Donc l'apparence juridique n'est rien» concluent $A$. Césaire, et avec lui les « Antilles Françaises».

Les Antilles Hollandaises: Iles de moindre importance où, selon le mot de la Reine Wilhelmine en 1954, «le colonialisme a disparu». En fait, sur une population très diverse de 180.000 habitants, le colonialisme s'est «assoupli » mais n'a pas disparu, les îles jouissent de l'« autonomie », leur parlement est élu au suffrage universel, mais elles demeurent propriété de la Couronne, et le « Gouverneur du Royaume » nomme magistrats et administrateurs.

[107]

\section{Vers une confédération caraibe}

Telle est, rapidement esquissée, l'évolution politique actuelle des Antilles sur la voie de l'indépendance.

Une conscience nationale antillaise est née: ça et là, des craquements se produisent dans les vieux cadres, mais une révolution complète et généralisée ne semble pas possible et nécessaire dans l'immédiat.

Dans cette situation, les leaders politiques estiment plus sage que chaque peuple commence par obtenir son indépendance dans le cadre où il se trouve, afin que la fédération de toutes les Antilles ne soit pas une construction rapide, artificielle et fragile, mais une confédération d'Etats majeurs, décidés à s'aider et à défendre mutuellement leur liberté. 


\section{Quelques chiffres}

États indépendants :

- Cuba

6.000.000 habitants

- Haïti

3.500 .000 habitants

- République dominicaine

2.300.000 habitants

Colonies ou « Possessions»:

- Antilles françaises (Martinique et 600.000 habitants Guadeloupe surtout)

- Antilles hollandaises (Curaçao)

180.000 habitants

- Porto-Rico (U.S.A.)

2.500.000 habitants

- Antilles anglaises

3.000 .000 habitants

Jamaïque

1.700 .000 habitants

Trinité 800.000 habitants

La Barbade 300.000 habitants

Iles du vent et Iles sous le vent 200.000 habitants 
Jacques Roumain, Poète Haittien, mort en 1945 :

Et bien voilà

Nous autres

Les Nègres

Les Niggers

Les sales Nègres

[108]

Nous n'acceptons plus

C'est simple

Fini

D'être en Afrique

En Amérique

Vos Nègres

Vos Niggers

Vos sales Nègres...

... Nous n'acceptons plus

Ça vous étonne

De dire : Oui missié

En cirant vos bottes

Oui mon pé

Aux missionnaires blancs

Oui maître

En récoltant pour vous

La canne à sucre

Le café

Le coton

L'arachide

En Afrique

En Amérique

En bons nègres

En pauvres nègres

Que nous étions

Que nous ne serons plus... 


\section{Pour la révolution africaine \\ Écrits politiques}

Quatrième partie. Vers la libération de l'Afrique.

\section{Chapitre 6}

\section{Le sang maghrebin ne coulera pas en vain ${ }^{21}$}

\section{Retour à la table des matières}

Il y a un peu plus d'un an, à l'annonce de l'interception de l'avion où avaient pris place les représentants du F.L.N. à la conférence maghrébine de Tunis, on pouvait voir dans les rues d'Alger ou de Paris des Français s'embrassant de joie et d'enthousiasme.

Le 8 février 1958, à la veille de la venue à Tunis de sa Majesté Mohammed $V$ invitée par le Président Bourguiba afin de faire le point de la question algérienne, une flotte aérienne composée de 25 avions a déversé sur le village de Sakiet Sidi Youssef une gerbe de bombes, de rocketts et de balles de mitrailleuses, tuant près de 100 civils, en blessant plus de 200 et détruisant la presque totalité du village.

21 El Moudjahid, № 18, 15 février 1958. 
Les différentes incursions des forces françaises sur le territoire tunisien à l'occasion desquelles des dizaines de Tunisiens trouvèrent la mort avaient soulevé l'indignation du peuple. A chacune de ces incursions les Tunisiens et les Tunisiennes prenaient davantage conscience du caractère précaire de leur indépendance. Cette précarité prenait sa racine d'abord dans le conflit franco-algérien, ensuite dans l'implantation des forces militaires françaises sur le territoire national. Plusieurs fois le Président Bourguiba avait demandé au gouvernement français l'ouverture de négociations en vue de l'évacuation de [110] ses troupes, chaque fois les responsables français provoquaient des incidents, créaient une tension et différaient la discussion générale sur le départ de l'armée française. Avec Sakiet Sidi Youssef le peuple tunisien s'est convaincu que non seulement, les Français entendent le «punir » de sa solidarité avec le peuple algérien mais encore espèrent prendre prétexte de cette solidarité pour reconquérir la Tunisie, prouvant ainsi, une fois pour toutes, que le Maghreb est un et qu'il doit être dominé par l'impérialisme français.

C'est pourquoi les Tunisiens et les Tunisiennes n'ont pas eu besoin d'exhiber leur colère ou de crier leur détermination. Pendant quatre jours, dans un calme impressionnant, le peuple affronté à son destin, après avoir prévu tous les risques qui menacent un peuple qui entend demeurer libre a décidé que Sakiet Sidi Youssef serait le dernier geste du colonialisme français en Tunisie. Ce qui veut dire que pendant ces quatre jours de réflexion, les Tunisiens et les Tunisiennes, placés une fois de plus en face d'une option fondamentale, ont réaffirmé leur serment fait il y a plusieurs années d'extirper de ce pays les dernières séquelles du colonialisme français. Ce qui veut dire aussi que le peuple tunisien derrière le Président Bourguiba a décrété l'état d'urgence. Le mot d'ordre, le principe vital aujourd'hui pour le peuple tunisien est l'évacuation totale du territoire national par les occupants colonialistes français.

On n'a pas suffisamment réfléchi à la rencontre rigoureuse des deux expressions les plus usitées depuis le 8 février: "évacuation», « des armes». Le peuple tunisien n'ignore pas que les Français ne sont pas près de quitter leurs casernes "gentiment ». Les Tunisiens savent qu'encore une fois, il faudra pousser les soldats français à la mer.

On a dit que les barrages sur la route étaient légers, qu'ils étaient fragiles, symboliques. On a ironisé sur la présence de fusils de chasse, 
de jeunes néo-destouriens désarmés : les journalistes français accrédités à Tunis n'ont de cesse qu'ils ne démontrent le caractère inefficace et somme toute illusoire des mesures prises par le peuple tunisien. Or, il y a un raisonnement qui se trouve frappé de nullité dans les pays coloniaux. C'est le raisonnement des crosses ou des tanks. Depuis pas mal de temps, l'argument d'autorité a crevé dans tous les pays coloniaux.

Le peuple tunisien a pris l'engagement devant son pays [111] et devant son drapeau de ne pas quitter la rue, de ne pas se reposer avant que le dernier soldat ait évacué le territoire national. Cela il faut que tout Français le sache. Il n'est plus possible que des troupes étrangères, ennemies, qui mettent en danger le régime intérieur et les assises de la nation, se maintiennent dans le pays, contre la volonté populaire.

En France, beaucoup de gens se sont embrassés. Les journaux français qui se vendent à plus d'un million d'exemplaires ont estimé que les Tunisiens n'avaient que ce qu'ils méritaient, que c'était tant pis pour Bourguiba et qu'à la réflexion ceci n'était qu'un début. Il faut reconnaître que dans les milieux officiels les premières réactions exprimèrent une certaine gêne. M. Pineau donna une fameuse interview à version double, tandis que M. Gaillard était soudain grippé.

Mais cette hésitation devait rapidement faire place au plus extraordinaire étalage d'agressivité et de bellicisme auquel on ait assisté depuis longtemps. Devant le parlement, M. Gaillard rejeta la responsabilité sur $M$. Bourguiba et $M$. Pineau n'hésita pas à menacer la Tunisie de l'escadre française de Toulon si les troupes continuaient à être gênées dans leurs mouvements.

À Alger, les Français invitent le gouvernement à poursuivre ses raids de représailles et en tout cas, estiment que l'aviation française ne saurait tolérer plus avant les insultes faites à son drapeau.

Certes, il y eut des gens en France pour regretter Sakiet Sidi Youssef, mais ces regrets sont circonstanciés. C'est une erreur, a-t-on pu dire, ou une faute. Certains ont insisté sur l'inopportunité de la chose. D'autres sur le fait qu'il fallait faire attention à la Croix Rouge, etc. Il y a enfin les autres regrets, ceux qui sont sincères, mais d'une sincérité hélas inefficace.

De toute façon, ce que le peuple tunisien réclame, ce ne sont pas des regrets. Ce que les Tunisiens et les Tunisiennes réclament ce ne 
sont pas des indemnisations pour les victimes de Sakiet Sidi Youssef: il y a des hommes, des femmes et des enfants tombés sous les coups du colonialisme pour que vive dans l'indépendance et la liberté le Maghreb unifié.

La décision du gouvernement tunisien de porter le crime [112] de Sakiet devant le Conseil de Sécurité exprime comme il se doit la profondeur de la volonté tunisienne.

Puisqu'il est prouvé maintenant aux yeux de l'opinion internationale que l'armée française, qui ne s'illustre que par les rapines ou les massacres des civils entend servir de moyen de pression sur le gouvernement tunisien en menaçant constamment l'indépendance nationale, il revient au Conseil de Sécurité de dire s'il acceptera qu'une armée étrangère occupe un pays contre sa volonté.

L'opinion mondiale dans son immense majorité n'a pas hésité à condamner l'agression française. Les Américains, esclaves de leur folie manichéiste, tremblent depuis huit jours que la Tunisie «ne sombre dans le nassérisme ». Et l'on voit les journalistes américains interroger à droite et à gauche sur les risques de ce revirement.

Il faut que les Américains sachent que s'ils veulent lutter contre le communisme ils doivent, dans certains secteurs, adopter des attitudes communistes. Pour les peuples coloniaux asservis par les nations occidentales, les pays communistes sont les seuls qui aient en toute occasion pris leur défense. Les pays coloniaux n'ont pas à se préoccuper de savoir si cette attitude est dictée par l'intérêt de la stratégie communiste, ils retiennent surtout que ce comportement général va dans le sens de leurs intérêts propres.

Les peuples coloniaux ne sont pas spécialement communistes mais ils sont irréductiblement anti-colonialistes.

On ne choisira pas les Etats-Unis parce qu'ils ont peur du communisme, mais parce que leur attitude dans les grands problèmes qui bouleversent le monde contemporain, en l'occurrence les problèmes de décolonisation, sera conforme à un esprit de solidarité, d'équité et de justice authentique.

Le peuple algérien, n'en déplaise à certains infirmes du cœur ou de l'esprit, ne jubile pas parce que Sakiet Sidi Youssef a été bombardé. Nous ne cherchons pas à exploiter cet événement. Nous sommes fon- 
damentalement opposés à la politique du pire. C'est avec émotion et avec douleur que nous avons appris l'épouvantable tuerie de Sakiet Sidi Youssef.

Nulle mort d'homme n'est indispensable au triomphe de la liberté. Il arrive qu'il faille accepter le risque de la mort pour que naisse la liberté, mais ce n'est pas de gaité de cœur que l'on petit assister à tant de massacres et à tant d'ignominies. [113] Bien que le peuple algérien fasse l'expérience quotidienne des B-26 français, il a été ébranlé par la tragédie de Sakiet Sidi Youssef.

Le Comité de Coordination et d'Exécution du F.L.N. a offert au peuple tunisien d'engager à ses côtés toutes les troupes disponibles pour l'aider à bouter hors de Tunisie l'occupant français.

Nous maintenons cette offre et nous disons au peuple tunisien, que nous sommes ensemble pour le meilleur et pour le pire et que le sang maghrébin est suffisamment généreux et qu'il s'offre à grandes ondées pour que d'Algérie à Sfax, il ne se trouve plus de soldats français pour menacer, torturer et massacrer. 


\section{Pour la révolution africaine \\ Écrits politiques}

Quatrième partie. Vers la libération de l'Afrique.

\section{Chapitre 7}

\section{La farce qui change de camp 22}

\section{Retour à la table des matières}

Depuis près de deux mois, nous assistons à la tentative vraiment forcenée des Anglo-américains pour transformer la tragédie de Sakiet Sidi Youssef, prolongement de la plus grande tragédie algérienne, en une farce où l'indécence se mêle à l'absurde.

Après Sakiet, la Tunisie porte plainte contre la France au Conseil de Sécurité. Dans un deuxième temps, la République tunisienne, dans une note conjointe, soumet le problème algérien à l'instance internationale. Sakiet exigeait du peuple tunisien des options précises : évacuation des troupes françaises, y compris Bizerte; restitution des aérodromes et, sur le plan international, intervention diplomatique en vue du conflit algérien.

Les Anglo-américains, exerçant un chantage à la solidarité occidentale, promettent au gouvernement tunisien d'obtenir de la France le respect de tous ces points sous réserve que les nations communistes

22 El Moudjahid, № 21 du $1^{\text {er }}$ avril 1958. 
ne soient pas intéressées, par le truchement du Conseil de Sécurité aux « problèmes intérieurs » atlantiques.

On a vu pendant un mois MM. Murphy et Beeley rencontrer, discuter. "Nous voulons rapprocher les points de vue français et tunisiens », prétendaient-ils et, pendant ce temps, le gouvernement français un moment ébranlé, reprenait son argumentation belliciste. En Algérie, les massacres continuaient [116] et le peuple algérien se trouvait face à 87 morts, un village rasé et des barrages qui répondent de moins en moins à la réalité de son drame et de sa colère.

Le Président de la République tunisienne a apporté certaines modifications aux exigences du peuple tunisien. Mais, au moment où le Chef du gouvernement tunisien annonçait ces concessions et en précisait les limites, le Président du Conseil français M. Gaillard présentait d'autres exigences, réclamait des garanties strictes, mettait le peuple tunisien au pied du mur, somme toute relançait la politique d'agressivité, de menace, d'intimidation.

Or, il y a un point sur lequel il n'est pas inutile d'attirer l'attention. C'est le fait que M. Dulles ait pu déclarer: «Après les concessions du gouvernement tunisien, la parole est à la France».

Nous avons dit plus d'une fois qu'avec le colonialisme il était vain d'espérer un quelconque assouplissement. Le colonialisme français est une force de guerre, et il faut l'abattre par la force. Nulle diplomatie, nul génie politique, nulle habileté ne pourront en venir à bout. Incapable qu'il est de se renier, il faut que les forces démocratiques s'allient pour le briser.

Quant à nous, Algériens, nous avons au cours de nos années de lutte amené à l'existence un certain nombre de vérités qui ont balayé les mensonges historiquement établis. Pour nous, Algériens, le triomphe de la démocratie ne dépend pas uniquement du monde occidental puisqu'aussi bien c'est ce même monde occidental qui en conteste les valeurs.

MM. Murphy et Beeley, par le jeu subtil de silences prolongés et d'un optimisme paradoxal, tentent de jeter la confusion dans le monde anti-colonialiste. Ils doivent savoir qu'ils tromperont peut-être beaucoup de monde, mais pas le peuple algérien. 
MM. Murphy et Beeley ne transformeront pas la lutte du peuple maghrébin pour l'indépendance en farce.

Les plénipotentiaires anglo-américains doivent abandonner tout espoir de jouer avec le peuple maghrébin à la manière de montreurs de marionnettes.

Il reste aux peuples marocain et tunisien à s'opposer avec ténacité aux manœuvres des porte-paroles du gouvernement français, délégués par le monde atlantique.

La tragédie de Sakiet, la tragédie du génocide perpétré [117] en Algérie par le peuple français occidental et chrétien, ne seront pas transformées en comédie burlesque où n'importe qui dit n'importe quoi, étant entendu que le dernier mot doit rester au « plus fort».

C'est la théorie même de la force traditionnelle des pays mercenaires qui sombre dans le ridicule.

Si la farce doit être jouée, elle sera jouée par la France. C'est la France, son régime et son peuple qui en feront les frais. 


\section{Pour la révolution africaine \\ Écrits politiques}

Quatrième partie. Vers la libération de l'Afrique.

\section{Chapitre 8}

\section{Décolonisation et indépendance ${ }^{23}$}

\section{Retour à la table des matières}

Depuis plus de trois ans la France «tient » en Algérie. Elle tient de la façon la plus opiniâtre, la plus exacerbée, au point que la mémoire de Clemenceau ne fut jamais, aux heures désastreuses de 1940, autant évoquée.

La France a perdu pied en Tunisie et au Maroc, mais s'est accrochée au territoire algérien. Pour des raisons diverses, l'éventail de l'opinion publique française, à de rares exceptions, a honoré l'armée, revendiqué la guerre d'Algérie, mis en garde les différents gouvernements contre d'inacceptables abandons en Algérie.

En dépit de la lassitude qui, épisodiquement, gagne les sphères politiques françaises au lendemain des quarts d'heure infiniment reportés de M. Lacoste, malgré, une crise budgétaire de plus en plus alarmante, malgré l'éclatement, autour de la guerre d'Algérie, de la presque totalité des partis politiques, on peut s'étonner qu'après trois ans, aucune

23 El Moudjahid, N²2 du 16 avril 1958. 
force cohérente et efficace n'ait vu le jour pour imposer la paix aux colonialistes français.

On n'a pas cessé de nous reprocher notre diplomatie à arêtes vives. Hô Chi Minh, nous rappelle-t-on, aux heures les plus tragiques de la guerre d'Indochine, n'a jamais cessé de faire la différence entre le colonialisme et le peuple français. Il n'est pas jusqu'aux exemples du Président Bourguiba et [120] des frères de l'Istiqlal qui ne soient évoqués pour nous inviter à composition.

Or, il ne faut pas oublier, puisqu'aussi bien on parle de l'Indochine, que c'est la décision d'y expédier le contingent qui provoqua la chute du gouvernement Laniel, la coalition des forces de gauche et le rendezvous de Genève.

Il est vrai qu'il y eut Dien Bien Phu. Mais le dernier livre de M. Laniel et les fracassantes déclarations d'anciens généraux d'Indochine soutiennent que, malgré Dien Bien Phu, si les «liquidateurs "n'avaient pas trahi la nation -entendez: ne s'étaient pas opposés à l'envoi du contingent, - l'Indochine aurait pu être sauvée.

Ce qu'il faut dire, c'est qu'avec la guerre d'Algérie sont apparus trois phénomènes, absolument nouveaux dans les luttes de libération nationale.

\section{Revendication et non prière}

Et d'abord à aucun moment le F.L.N. n'a fait appel à la générosité, à la magnanimité, à la gentillesse du colonisateur. Le colonisé accède dans une mutation vertigineuse à une qualité nouvelle, élaborée dans et par le combat. Le langage utilisé par le F.L.N., dès les premiers jours de la Révolution, est un langage de responsable. Les appels aux forces démocratiques française ne sont pas rédigés en termes ambigus de teinte plus ou moins infantile. Nous disons à la gauche française: soyez logiques avec vous-mêmes, - aidez-nous, soutenez la cause de la Révolution algérienne. Le souci constant du F.L.N. de débarrasser les rapports colonisé-colonisateur, peuple algérien-peuple français, du confusionnisme traditionnel a mis les éléments démocratiques français dans une situation inhabituelle. Leur action est invitée à s'incarner, 
moins dans une atmosphère de sympathie diffuse que dans la rigueur doctrinale d'un anticolonialisme authentique. Pareille délimitation des frontières pouvait, après un examen superficiel, être assimilée à de la rigidité. C'est pourquoi il n'est pas rare d'entendre les démocrates français nous rétorquer: Si vous continuez on vous laisse tomber. Une telle position indique que l'action des démocrates est dépourvue de toute valeur révolutionnaire et doctrinale, car précisément elle s'alimente aux sources ambivalentes de la bonté pour l'opprimé ou de la soif de faire quelque chose, d'être utile, etc...

On a reproché au F.L.N. de ne pas savoir parler aux Français, de ne pas suffisamment compter avec leurs petites faiblesses et leur narcissisme. Cela est certainement vrai.

Mais ces reproches indiquent que l'objectif du F.L.N. est à peine retenu. Le F.L.N. ne vise pas à réaliser une décolonisation de l'Algérie ou un assouplissement des structures oppressives.

$\mathrm{Ce}$ que le F.L.N. réclame, c'est l'indépendance de l'Algérie. Une indépendance qui permette au peuple algérien de prendre totalement son destin en main.

Cet objectif, cette stratégie, commandent notre tactique, notre méthode et informe l'allure même de notre lutte.

\section{Le colonialisme est fondamentalement inexcusable}

La Révolution algérienne a introduit un scandale dans les déroulements des luttes de libération nationale. En général le colonialisme, au moment où l'histoire et la volonté nationale le nient, parvient à se maintenir comme vérité et valeur. Il n'est pas vrai que ce fut une bonne chose que la France ait fait de l'Algérie ce qu'elle est aujourd'hui.

Le port de Mers El Kebir et l'aérodrome pour avions à réaction de Boufarik ne nous consoleront jamais de la grande misère intellectuelle, morale et matérielle de notre peuple. 
Le colonialisme français ne sera pas légitimé par le peuple algérien. Nulle entreprise spectaculaire ne nous fera oublier le racisme légal, l'analphabétisme, le larbinisme suscité et entretenu au plus profond de la conscience de notre peuple.

C'est pourquoi il n'est jamais question dans nos déclarations d'adaptation ou d'allègement, mais bien de restitution. Il est vrai que l'on n'a pas cessé de reprocher au F.L.N. cette constante référence à la nation algérienne d'avant Bugeaud. C'est qu'en insistant sur cette réalité nationale, en faisant de la Révolution du $1^{\text {er }}$ novembre 1954 une phase de la résistance populaire commencée avec Abd El Kader, nous enlevons au colonialisme français sa légitimité, sa prétendue insertion dans la réalité algérienne. Au lieu d'intégrer le colonialisme, conçu comme naissance d'un monde nouveau, dans l'histoire algérienne, nous en avons fait un accident malheureux, exécrable, dont la seule signification était d'avoir retardé de façon [122] inexcusable l'évolution cohérente de la société et de la nation algériennes.

La «Nation en formation», "l'Algérie nouvelle», «le cas unique dans l'histoire», toutes ces expressions mystificatrices ont été balayées par la position du F.L.N. et il n'a subsisté en plein soleil que le combat héroïque de tout un peuple contre l'oppression séculaire.

Entre la rupture avec le passé algérien avec, comme conséquence, l'installation dans une colonisation rénovée mais continuée et la fidélité à la nation transitoirement asservie, le peuple algérien a choisi :

Il n'y a pas une entité nouvelle née du colonialisme. Le peuple algérien n'a pas accepté qu'on transforme l'occupation en collaboration. Les Français en Algérie n'ont pas cohabité avec le peuple algérien. Ils ont peu on prou dominé. C'est pourquoi il fallait dès le début faire sentir au peuple français la portée de nos revendications.

Le F.L.N. n'a pas joué sur les mots. Il a dit que son objectif était l'indépendance, qu'aucune concession ne pouvait être faite sur cet objectif. Le F.L.N. a dit aux Français qu'il fallait négocier avec le peuple algérien, lui restituer son pays, tout son pays.

Dès le début, le F.L.N. a précisé son programme: mettre un terme à l'occupation française, donner les terres aux Algériens, faire une politique de démocratie sociale où l'homme et la femme ont droit également à la culture, au bien-être matériel et à la dignité. 


\section{C'est un individu libéré qui entreprend la construction de la cité}

Une telle attitude devait rapidement avoir sur la conscience de l'Algérien des répercussions importantes.

Toutes les structures abêtissantes et infantilisantes qui infestent habituellement les relations entre le colonisé et le colonisateur se sont trouvées brutalement liquidées. Alors que le colonisé le plus souvent n'a de choix qu'entre une rétraction de son être et une tentative forcenée d'identification au colonisateur, l'Algérien a amené à l'existence une personnalité [123] nouvelle, positive, efficiente, dont la richesse s'alimente moins dans l'épreuve de force qu'il assume que dans sa certitude d'incarner un moment décisif de la conscience nationale.

Le combattant algérien n'est pas seulement dressé contre les parachutistes tortionnaires. Il est la plupart du temps confronté avec des problèmes d'édification, de construction, d'invention de la cité. C'est pourquoi irréversiblement, le colonialisme a perdu la partie en Algérie. A l'échelle de chaque wilaya des plans cadastraux sont établis, des projets de construction scolaire étudiés, des reconversions économiques poursuivies.

L'Algérien construit, organise, légifère, planifie. D'où son assurance, son langage ferme et résolu, la cohésion énergique de ses positions.

On comprend pourquoi les porte-paroles du F.L.N. sont généralement décrits comme intransigeants. Ce n'est pas le ton qui indispose mais le contenu de nos revendications.

Beaucoup de peuples colonisés ont réclamé la fin du colonialisme, mais rarement comme le peuple algérien.

Ce refus de solutions évolutives, ce mépris pour les «étapes» qui brisent le torrent révolutionnaire et désapprennent au peuple cette volonté inébranlable de tout prendre en mains tout de suite afin que tout change, constituent la caractéristique fondamentale de la lutte du peuple algérien. 
Et le «Moudjahid» qui expose cette position, la défend et la fait triompher, introduit une donnée nouvelle dans le classique dialogue du dominé et de l'oppresseur. La libération de l'individu ne suit pas la libération nationale. Une authentique libération nationale n'existe que dans la mesure expresse où l'individu a amorcé irréversiblement sa libération. Il n'est pas possible de prendre ses distances à l'égard du colonialisme sans en même temps les prendre à l'égard de l'idée que le colonisé se fait de lui-même à travers le filtre de la culture colonialiste.

Une telle révolution à l'échelle de la conscience nationale et de la conscience individuelle devait être analysée. Elle permet de comprendre la déroute et la peur du colonialisme français en Algérie.

[124]

\section{La révolution algérienne introduit un nouveau style dans les luttes de libération nationale}

Il est un troisième phénomène non encore identifiable mais qui, atmosphériquement, bouleverse les relations de tous les colonisés avec la France. Le processus de libération des peuples coloniaux est certes inéluctable. Mais la forme donnée à la lutte du peuple algérien est telle, dans sa violence et dans sa totalité, qu'elle influence de façon décisive les luttes futures des autres colonies.

Le peuple algérien expérimente concrètement l'interdépendance des phénomènes historiques. Dire que l'écroulement localisé du colonialisme augmente sa désagrégation en tant que système n'est plus l'explication d'un principe abstrait seulement perceptible par les couches intellectuelles.

Tout le peuple algérien sait qu'après l'Algérie ce sera à l'Afrique Noire de mener son combat. Et n'est-il pas vrai que dès maintenant la France se trouve acculée à inventer de nouvelles formules, à voter la Loi-Cadre puis à la dépasser, s'acheminant malgré elle vers la reconnaissance de la souveraineté nationale de l'Afrique Noire? 
Certes, des hommes politiques africains prisonniers des Français tentent de suivre la courbe irrésistible de la revendication nationale.

Jusqu'à présent ils ont pu adopter avec suffisamment de célérité les différentes positions de leurs peuples. Mais tôt ou tard le divorce se manifestera. Il leur faudra alors être « traîtres» en plein jour ou abandonner les mirages de l'œuvre colonisatrice.

Le peuple algérien sait que les peuples d'Afrique Noire suivent avec sympathie et enthousiasme sa lutte contre le colonialisme français. Le peuple algérien n'ignore pas que chaque coup porté contre l'oppression française en Algérie démantèle la puissance colonialiste.

Chaque embuscade tendue, chaque garnison investie et détruite, chaque avion abattu sème la panique dans le dispositif colonial français et renforce la conscience nationale africaine ou malgache ou antillaise.

Les peuples opprimés savent aujourd'hui que la libération [125] nationale est inscrite dans le développement historique mais ils savent aussi que cette libération doit être l'œuvre nécessaire du peuple opprimé.

Ce sont les peuples coloniaux qui doivent se libérer de la domination colonialiste.

La véritable libération n'est pas cette pseudo-indépendance où les ministres à responsabilité limitée voisinent avec une économie dominée par le pacte colonial.

La libération est la mise à mort du système colonial, depuis la prééminence de la langue de l'oppresseur et la « départementalisation», jusqu'à l'union douanière qui maintient en réalité l'ancien colonisé, dans les mailles de la culture, de la mode et des images du colonialiste.

Cette mise à mort, le peuple algérien l'a entreprise avec tenacité et ferveur.

Nous n'attendons pas que ce colonialisme se suicide. Il est dans sa logique de se défendre avec acharnement. C'est d'ailleurs la prise de conscience de son impossibilité à se survivre qui déterminera sa liquidation en tant que style de contact avec les autres peuples. 
Le peuple colonialiste ne sera guéri de son racisme et de son infirmité spirituelle que si, réellement, il accepte de considérer l'ancienne possession comme une nation absolument indépendante.

Toute évocation de « liens anciens » ou de «communautés » irréelles est un mensonge et une ruse.

Le peuple algérien prouve depuis près de quatre ans que ce mensonge et cette ruse ne sont plus à la mesure de sa vérité et de sa volonté. 


\section{Pour la révolution africaine \\ Écrits politiques}

Quatrième partie. Vers la libération de l'Afrique.

\section{Chapitre 9}

\section{Une Crise continuée 24}

\section{Retour à la table des matières}

Pour la quatrième fois depuis le $1^{\text {er }}$ novembre 1954, la France se trouve sans gouvernement.

Après les équipes Edgar Faure, Guy Mollet, Bourgès-Maunoury et la dernière en date, dite d'union nationale de M. Félix Gaillard, voici une nouvelle crise que les uns et les autres s'accordent à juger extrêmement grave.

Et certes, personne ne saurait ignorer aujourd'hui que la guerre d'Algérie est à l'origine de cette instabilité gouvernementale en France. Toutefois il faut tenter de saisir le développement interne du processus responsable de ce déséquilibre. Il faut éclairer le travail de détérioration et de désagrégation du prestige français qui se poursuit hors de France et en France à propos de ces guerres coloniales.

24 El Moudjahid, No 23 du 5 mai 1958. 
L'analyse de la situation doit nous permettre d'apprécier, de mesurer cette fatalité interne qui mine la France et qui la conduit presque mécaniquement à multiplier les crises, a s'enfermer dans une atmosphère de crise.

Dire que la guerre d'Algérie épuise les finances et déséquilibre l'économie de la France c'est énoncer évidemment une vérité. Mais on aurait tort de privilégier cette vérité. On a pu s'en rendre compte à l'occasion des débats à l'Assemblée Nationale française sur le coût des opérations en Algérie. Tandis que les experts internationaux, en l'occurrence ceux qui [128] épisodiquement renflouent l'économie française, chiffraient les dépenses à 800 milliards, M. Lacoste prétendait sans humour que la guerre d'Algérie ne coûtait rien.

Les partis français de gauche, prisonniers d'un simplisme doctrinal qui veut que la droite négocie dès lors qu'on la convie à participer aux frais, se sont installés dans une attitude résignée et ne sont plus qu'attente du jour où cette droite, mise en danger dans ses intérêts, abandonnera son chauvinisme et votera toutes les indépendances coloniales que l'on voudra.

Nous avons, à plusieurs reprises, signalé cette mécanisation de la pensée et l'apparition de ce fétichisme des causes pris au sens le plus automatique, le moins dialectique.

Cette faiblesse idéologique d'une grande partie des formations politiques françaises éclaire le conflit qui existe dans le corps politique français depuis le $1^{\text {er }}$ novembre 1954. Le parti radical pulvérisé depuis l'historique congrès de Lyon, le parti socialiste au sein duquel des tendances de plus en plus hétérogènes apparaissent, le M.R.P. qui vient de révéler ses contradictions par son récent refus de cautionner la tentative de M. Bidault, jusqu'au P.C.F. que sa structure monolithique n'a pas préservé des divergences et des oppositions sur le thème de la guerre d'Algérie, chacun de ces partis, selon son style propre, manifeste l'incohérence des positions, la violence des conflits, le désaccord fondamental. 


\section{Les contradictions européennes}

La pire erreur serait d'ailleurs d'isoler cette destructuration de la vie politique française du contexte européen et international.

Les nations européennes, surtout I'Italie, l'Angleterre et l'Allemagne occidentale, pour des raisons de compétition économique en Europe ou de débouchés à préserver en Afrique, ont senti la nécessité de manifester une hostilité implicite aux guerres coloniales menées par la France.

Pour prendre un exemple, le peuple italien qui a pendant longtemps gardé le silence devant l'appui de son gouvernement à la guerre coloniale française, alerté par les formations démocratiques italiennes, s'est mis en mouvement depuis quelques mois et réclame de façon péremptoire le non-engagement du gouvernement italien aux côtés du colonialisme français.

[129]

En Allemagne occidentale, il est maintenant établi que pas un journal allemand n'ose soutenir la politique française. Les représentants français en Allemagne ont l'occasion de constater plusieurs fois par jour que l'ensemble du peuple allemand condamne le colonialisme français et porte sa sympathie au glorieux peuple algérien.

En Angleterre, les conservateurs, solidaires pendant longtemps des colonialistes français, ont depuis quelques mois amorcé le virage et leurs organes de presse ne dissimulent plus leurs positions sur la nécessité de négociations avec le peuple algérien.

$\mathrm{Ce}$ changement d'orientation des démocraties occidentales est la conséquence d'une part d'un courant anti-colonialiste et libéral certain, mais surtout, il ne sert à rien de le cacher, du désir de voir une France ramenée à sa seule dimension européenne, amputée de ses colonies, privée des régimes préférentiels instaurés par le pacte colonial et confrontée enfin sur le plan de la libre concurrence avec les autres économies nationales européennes. Dans cette seconde perspective, à la formule "l'Afrique, chasse gardée de la France», tend à se substituer la deuxième formule, «l'Afrique, chasse gardée de l'Europe ». 


\section{Les contradictions occidentales et les pressions internationales}

Les Etats-Unis d'Amérique, face au monde communiste, développent une politique africaine qui rejoint dans ses fondements les nouvelles positions européennes. Les démocrates américains, quand ils exposent leur position sur la décolonisation de l'Afrique, insistent toujours sur la nécessité pour les Etats-Unis de ne pas partager la perspective colonialiste française.

Le thème dominant de leur argumentation est net: en Afrique, la France est en train de compromettre les chances de l'Occident et de «l'homme blanc».

Toutefois, deux autres éléments circonstancient la progressive modification de l'attitude américaine. Et d'abord la certitude aiguë, outre-atlantique, que l'heure est venue de faire un choix, de soutenir la lutte d'indépendance des peuples coloniaux, d'aider à la désagrégation des empires, d'appuyer la libération des peuples opprimés. En outre, face au « danger [130] communiste», dans le cadre de la guerre froide et du partage du monde en sphères d'influence, les Etats-Unis sont de plus en plus conscients de l'urgence des positions radicalement opposées au colonialisme français.

Sur le plan international, il vaut la peine d'insister sur l'immense enthousiasme soulevé dans les pays afro-asiatiques par l'épopée que vit depuis près de quatre ans le peuple algérien.

Bandoeng, le Caire, Accra, tous les peuples afro-asiatiques tous les opprimés d'hier, portent, soutiennent et assument de plus en plus la cause de la Révolution algérienne: il n'est absolument pas exagéré de dire que, de plus en plus, la France aura contre elle, en Algérie, deux continents.

C'est faute d'avoir analysé ces multiples facteurs, ces contradictions dialectiques, que les partis politiques français se trouvent le plus souvent dans une situation d'indétermination, d'exacerbation passionnelle sans thème directeur, d'inquiétude, toutes choses qui ne sont pas sans évoquer des conduites d'auto-destruction. 


\section{L'atmosphère de crise}

En France, le refus d'un choix politique cohérent en Algérie ne concerne pas uniquement les groupes politiques.

Au sein de chaque parti on peut distinguer aujourd'hui un centre, une droite et une gauche. Au niveau des masses populaires ou de corps particuliers comme l'Eglise, on est frappé par une ambiance de malaise d'amertume et de désespoir. Pas une association, pas un groupe d'hommes ou de femmes qui ne soit atteint et affecté par le développement de la guerre d'Algérie: scission des associations d'étudiants, scission des enseignants, bouleversement dans l'armée, rappel à l'ordre des cardinaux, malaise dans la police. Tous ces phénomènes, par leur nombre et leur gravité, indiquent le désarroi qui règne dans la vie morale et politique en France.

La volonté de libération du peuple algérien conteste à n'en pas douter la fiction de l'Algérie française. Mais c'est aussi un certain type de comportement, un style de contact intellectuel qui se trouve condamné de part en part. Le combat du peuple algérien est une critique radicale du pseudo-droit de propriété : notre Afrique noire, notre Algérie... et [131] en même temps une mise en demeure du peuple français à se critiquer, à se débarrasser de la mentalité colonialiste, antidémocratique et raciste, bref à vivre et à dépasser des contradictions historiquement élaborées.

\section{La lutte du peuple algérien, source de vérité}

Or, le désarroi qui règne actuellement dans la vie morale et politique française serait incompréhensible si on ne le rattachait dialectiquement aux réalités internationales et à la lutte du peuple algérien.

Les critiques décisives et implacables du sénateur Kennedy les prises de position profondément anti-colonialistes des Travailleurs an- 
glais et, d'une manière plus générale, la récente prise de position des services officiels américains, traduisent deux phénomènes. Et d'abord que le processus historique et général de libération des peuples coloniaux est reconnu, identifié et accepté mais aussi la certitude acquise après l'analyse que le peuple algérien a jeté tout son poids dans la lutte et que véritablement on ne voit pas comment la France pourrait ne pas reconnaître l'indépendance de l'Algérie.

M. Lacoste n'est pas devenu impopulaire dans le monde et dans l'opinion française parce qu'il a dirigé la répression ou justifié les assassinats collectifs. C'est que le mensonge de M. Lacoste est apparu en pleine lumière, trahi et démasqué par l'invincibilité de l'Armée nationale algérienne. Les «quarts d'heure » indéfiniment rapportés, les bulletins de victoire totale, les proclamations fantaisistes, le bluff, expliquent la confusion de l'ambivalence qui règne autour de son nom. La gauche le condamne parce qu'il s'oppose à une politique de gauche, et la droite parce qu'il n'a pas tenu ses promesses parce qu'il n'a pas pacifié l'Algérie, parce que la situation militaire devient de plus en plus grave.

Donc, il n'existe pas une crise politique française parce que des opinions se font face ou que des conceptions du monde divergent, mais bien parce que le scandale que constitue la volonté de libération du peuple algérien bouleverse les équilibres établis, les vérités admises et remet totalement en question les perspectives de la nation française.

La crise française ne se développe pas en milieu fermé. Ce n'est pas une crise de la réflexion pure. C'est dans la pratique [132] la plus tragique que le peuple français repense et reconstruit son système de valeurs.

En intensifiant son effort militaire, le peuple français a imposé au peuple algérien des sacrifices immenses, mais précisément le développement dialectique de ce combat devait provoquer en retour un bouleversement idéologique en France et faire éclater l'évidence que l'effort français allait à l'encontre de l'histoire, de la morale, de I'humain.

C'est la lutte acharnée, opiniâtre, héroïque, du peuple algérien qui fait apparaître de nouvelles significations, dépasse certaines contradictions, rend possible ce qui était impensable hier.

Les déclarations de pré-investiture de M. Bidault n'ont pas mécontenté et irrité parce qu'elles étaient mal rédigées. C'est que de 
toute évidence M. Bidault signifiait qu'il n'avait rien compris, qu'il n'avait pas suivi les événements, qu'il était déconnecté de l'histoire, qu'il était en dehors du temps. Ce temps nouveau, ces conditions nouvelles, ces réalités originales sont précisément introduites par le combat du peuple algérien.

C'est la lutte du peuple algérien qui a amorcé cette transformation de la vie politique française. C'est au contact de cette volonté nationale, de cette violence dans la revendication, que se remodèlent les perspectives politiques françaises.

Pressions européennes, parce que tout de même, l'Afrique, si elle est perdue pour la France, ne devrait pas l'être pour l'Europe. Pressions internationales car l'hydre communiste risque de s'installer en Afrique si l'ensemble de l'Occident se maintenait dans ce silence complice devant le bellicisme français. Pressions du peuple algérien, élément le plus actif dans cette dialectique et aussi le plus vrai. Ces trois ordres de facteurs cernent la réalité nationale française et induisent, suscitent et démasquent les contradictions constitutives d'un pays colonialiste et raciste à exigences doctrinales paradoxalement démocratiques.

Et il n'est pas vrai que cette confrontation puisse s'arrêter magiquement. Il n'est pas vrai que l'Amérique ou l'Italie puissent être mises au pied du mur. Il n'est pas vrai que M. Pineau puisse, s'il le demandait, obtenir l'appui de l'O.T.A.N. Il n'est pas vrai qu'avec un peu de bonne volonté $M$. Mendès-France puisse renouer avec M. Morice ou M. André Philip fraterniser avec M. Mollet. Il n'est pas vrai que l'école [133] d'état-major de $M$. Bigeard puisse apporter quelque chose de nouveau dans la guerre d'Algérie. Toutes ces impossibilités sont les négatifs d'une réalité majeure: la France est plongée dans une atmosphère de crise cataclysmique et elle n'en sortira que par la négociation avec le F.L.N. 


\section{Pour la révolution africaine \\ Écrits politiques}

Quatrième partie. Vers la libération de l'Afrique.

\section{Chapitre 10}

\section{Lettre à la jeunesse africaine 25}

\section{Retour à la table des matières}

Le peuple algérien, depuis quatre ans, mène, un combat très dur contre le colonialisme français. Vous n'ignorez pas l'effort colossal que la France a fourni en Algérie pour maintenir sa domination. Et vous savez que le F.L.N. qui dirige notre lutte a toujours opposé une résistance farouche à la guerre de reconquête française.

Nous nous sommes déjà à plusieurs reprises adressés aux pays coloniaux en général et il nous a toujours paru important de signaler la faillite de certaines données et l'apparition de thèses totalement inattendues.

Nous n'avons cessé de dire, depuis trois ans, que le coin enfoncé dans le système colonial français par la libération de l'Indochine, de la Tunisie et du Maroc en avait ébranlé l'infrastructure, mais qu'il ne fallait pas se faire d'illusions, la bête étant encore suffisamment robuste.

25 El Moudjahid, No24 du 29 mai 1958. 
Le monde colonial subit depuis une quinzaine d'années des assauts de plus en plus violents et l'édifice lézardé est en voie d'écroulement. Nul ne conteste aujourd'hui que cette liquidation du colonialisme ne constitue la marque spécifique de l'après-guerre. Le processus historique, né des multiples contradictions inhérentes au système capitaliste et dynamisé par la volonté nationale des peuples opprimés, préside à la naissance d'Etats indépendants.

[136]

Les peuples colonisés se sont généralement reconnus dans chacun des mouvements, dans chacune des révolutions mises en branle et menées à termes par les opprimés. Au-delà de la nécessaire solidarité avec les hommes qui, sur toute la surface de la terre, se battent pour la démocratie et le respect de leurs droits, s'est imposée, avec une violence inaccoutumée, la ferme décision des peuples colonisés à vouloir pour eux-mêmes et pour leurs frères, la reconnaissance de leur existence nationale, de leur existence en tant que membres d'un Etat indépendant, libre et souverain.

Depuis plusieurs années l'histoire du monde, l'histoire de la lutte des hommes pour la dignité, pose aux peuples des problèmes définis. Les hommes asservis et opprimés par des nations étrangères sont aujourd'hui invités à participer totalement à l'œuvre de démolition du système colonial. Et il n'est pas exagéré de dire que si les parties du monde où s'est déjà réalisée l'existence nationale marquent le pas sans dépasser leurs contradictions, c'est parce que précisément toute nouvelle marche vers le progrès implique la libération des colonies.

Il faut que les peuples opprimés rejoignent les peuples déjà souverains afin que soit valable l'édification d'un humanisme aux dimensions de l'univers.

Depuis dix ans, le devoir de tout colonisé est précis : sur le sol national, miner l'édifice colonialiste et soutenir de façon positive les luttes des peuples colonisés.

La guerre de libération du peuple algérien a étendu la gangrène et a porté le pourrissement du système à un tel degré qu'il est devenu évident pour les observateurs qu'une crise globale doit en résulter.

C'est en prévision de cette possible mutation, de cette éventuelle remise en question générale, que nous nous sommes adressés à maintes 
reprises à vos représentants aux Assemblées parlementaires françaises et à vos leaders syndicalistes.

Depuis trois ans nous n'avons cessé de les inviter à acculer la bête colonialiste, à l'obliger à desserrer son étreinte; depuis trois ans nous n'avons cessé d'expliquer, d'exposer à vos représentants qu'il fallait conjuguer leurs efforts et faire éclater l'empire français, puisqu'aussi bien le peuple algérien menait sur son territoire une guerre ouverte, grandiose et difficile.

Nous devons à la vérité de vous dire que presque tous vos représentants, mystifiés par un phénomène d'aliénation très grave, ont toujours opposé à nos démarches le respect de la légalité républicaine française.

Or, il semble qu'au moins trois points nous soient communs. Et d'abord nos nations respectives sont occupées militairement, exploitées économiquement et silencieuses culturellement depuis que le drapeau tricolore y flotte.

Toute, poussée vers une expression de soi-même conforme à son histoire, fidèle à sa tradition et liée à la sève même de son sol se trouve limitée, stoppée, brisée.

Le style du pacte colonial qui régit l'exploitation multidimensionnelle des territoires de "I'Union française », constitue notre deuxième point commun. Ce n'est pas assez de dire que la France occupe notre sol national, mais elle s'y est installée de manière désinvolte et n'a pas craint d'élaborer toute une législation, tout un code à partir de quoi notre essence nationale se trouve niée au profit de l'ordre français.

La volonté d'indépendance qui devait constituer l'unique réponse à cette colonisation est le troisième point commun des peuples dominés par la France. Quand nous nous adressons aux peuples coloniaux et plus spécialement aux peuples africains, c'est à la fois parce que nous avons à nous dépêcher pour construire l'Afrique, pour qu'elle s'exprime et se réalise, pour qu'elle enrichisse le monde des hommes et pour qu'elle puisse être authentiquement enrichie des apports du monde. C'est aussi parce que l'unique moyen de parvenir à ce résultat est de briser les reins au colonialisme le plus forcené, le plus intraitable, le plus barbare qui soit. 
À l'heure actuelle, tous les mouvements de libération des peuples coloniaux, quelles que soient par ailleurs les nations dominatrices, sont liés à l'existence du colonialisme français.

"L'Empire français», par son étendue, jouit encore aujourd'hui d'un certain prestige et d'une apparente stabilité. La hargne du colonialisme français, son mépris de la morale internationale, sa spectaculaire barbarie, rassurent les autres pays colonialistes.

Jeunesse des pays coloniaux!

Il faut que vous sachiez que l'avenir de votre existence [138] nationale, la cause de votre liberté et de votre indépendance se trouvent en jeu actuellement en Algérie.

Il n'est pas vrai, comme l'ont prétendu certains des «parlementaires " coloniaux siégeant dans les Assemblées françaises, que la guerre d'Algérie favorise le processus de décolonisation et qu'en conséquence il suffit d'exploiter sur le plan parlementaire ces difficultés du colonialisme français.

Il est vrai que la Loi-Cadre votée sur la pression de la guerre d'Algérie a amorcé un desserrement de l'étreinte sur les pays d'Afrique, mais nous pensons qu'il serait très grave d'attacher une valeur autre que caricaturale à cette «évolution».

$\mathrm{Ce}$ que veulent les pays coloniaux, ce n'est pas un «bon geste» du maître, mais très précisément la mort de ce maître. En outre, dans le cadre même de cette évolution, il est fréquent de constater la « mauvaise volonté » des administrateurs français, leur mouvement d'humeur contre la parcelle de liberté donnée aux «Nègres», leur rage devant cette atteinte à la suprématie du blanc. Et il faut souligner le comique de certains parlementaires coloniaux qui menacent de rappeler des administrateurs français hostiles à la Loi-Cadre.

Une saine analyse du colonialisme français à la quatrième année de la guerre d'Algérie aurait dû conduire ces parlementaires à envisager cette « mauvaise volonté » moins comme un fait individuel que comme l'expression d'un colonialisme encore très solide sur ces positions et comme le signe, qu'en France, tout serait mis en œuvre pour interdire une quelconque évolution des pays coloniaux et une quelconque atteinte au pacte colonial. 
Ce qui se passe aujourd'hui en France, en Algérie, appartient au même processus que les «mauvaises volontés d'administrateurs ou de colons.

Jeunesse des pays coloniaux!

Depuis quatre ans nous ne cessons de répéter à ceux qui siègent dans les Assemblées françaises que le colonialisme français ne fera l'objet d'aucune opération magique et qu'il est vain d'en espérer une progressive disparition.

L'avenir sera impitoyable pour ces hommes qui, jouissant du privilège exceptionnel de pouvoir dire à leurs oppresseurs des paroles de vérité, se sont cantonnés dans une attitude de [139] quiétude, d'indifférence muette et quelquefois de froide complicité.

M. Houphouët-Boigny, député africain et Président du R.D.A., a, il y a quelques jours, accordé une interview à la presse. Après des considérations absurdes sur l'évolution souhaitée d'une Afrique ceinte du drapeau tricolore, il en arrive à la question algérienne et n'hésite pas à affirmer que l'Algérie doit demeurer dans le cadre français.

Ce monsieur, depuis plus de trois ans, s'est fait l'homme de paille du colonialisme français. Siégeant dans tous les gouvernements, M. Houphouët-Boigny a assumé directement la politique d'extermination pratiquée en Algérie.

Ayant à sa droite M. Lacoste et MM. Morice ou Chaban-Delmas à sa gauche, M. Houphouët-Boigny a cautionné de façon impardonnable une politique qui a endeuillé la nation algérienne et compromis pour de longues années le développement de notre pays.

M. Houphouët-Boigny s'est fait le commis-voyageur du colonialisme français et il n'a pas craint de se rendre aux Nations-Unies pour y défendre la thèse française.

M. Houphouët-Boigny est docteur en médecine. Il était ministre de la Santé de M. Gaillard. C'est sous son règne qu'eut lieu Sakiet Sidi Youssef. Les ambulances de la Croix-Rouge Internationale y furent mitraillées, bombardées, éventrées. Des dizaines de femmes et d'enfants furent coupés en deux par les rafales de l'aviation française. 
L'Africain Houphouët-Boigny, le docteur en médecine HouphouëtBoigny, n'ont pas craint l'un et l'autre de revendiquer cette barbarie et de se déclarer solidaires des militaires français.

M. Houphouët-Boigny, en bon ministre de la République française, a estimé que son devoir était d'assumer Sakiet, de féliciter la vaillante armée française et d'appuyer en toute solidarité ministérielle les pressions sur le gouvernement tunisien.

Aux belles heures de l'impérialisme français, ce pouvait être une sorte d'honneur pour un colonisé de faire partie du gouvernement français. Cet honneur sans responsabilité ni risque, cette complaisance puérile à être ministre ou Secrétaire d'Etat, pouvaient à la rigueur être pardonnés.

Or, depuis dix ans, il est devenu proprement intolérable et [140] inacceptable que des Africains puissent siéger dans le gouvernement du pays qui les domine.

Tout colonisé qui accepte aujourd'hui un siège gouvernemental doit savoir de la façon la plus claire qu'il aura à cautionner, tôt ou tard, une politique de répression, de massacres, d'assassinats collectifs dans l'une des régions de «l'Empire français».

Lorsqu'un colonisé comme M. Houphouët-Boigny, oublieux du racisme des colons, de la misère de son peuple, de l'exploitation éhontée de son pays en arrive à ne pas participer à la pulsation libératrice qui soulève les peuples opprimés et que, en son nom, tous pouvoirs sont donnés aux Bigeard et autres Massu, nous ne devons pas hésiter à affirmer qu'il s'agit ici de trahison, de complicité et d'incitation au meurtre.

Jeunesse d'Afrique, de Madagascar, des Antilles, les militaires de vos patries respectives enrôlés de force dans l'armée française ont rejoint avec enthousiasme les rangs de l'Armée de Libération Nationale algérienne. Aujourd'hui, côte à côte avec les patriotes algériens, ils poursuivent une lutte héroïque contre l'ennemi commun.

Le F.L.N. qui dirige le combat du peuple algérien s'adresse à vous et vous demande de faire pression sur vos parlementaires pour les obliger à déserter les Assemblées françaises.

L'heure est venue pour tous les coloniaux de participer activement à l'éreintement des colonialistes français. 
Où que vous soyiez, il faut que vous sachiez que le moment est arrivé pour nous tous d'unir nos efforts et d'assener le coup de grâce à l'impérialisme français.

Jeunesse africaine! Jeunesse malgache! Jeunesse antillaise! Nous devons, tous ensemble, creuser la tombe où s'enlisera définitivement le colonialisme! 


\section{Pour la révolution africaine \\ Écrits politiques}

Quatrième partie. Vers la libération de l'Afrique.

\section{Chapitre 11 \\ Vérités premières à propos du problème colonial 26}

\section{Retour à la table des matières}

Le XXe siècle, à l'échelle, du monde, n'aura pas été seulement l'ère des découvertes atomiques et des explorations interplanétaires. Le deuxième bouleversement de cette époque est sans conteste, la conquête par les peuples des terres qui leur appartiennent.

Bousculés par la revendication nationale de régions immenses, les colonialistes ont dû desserrer leur étreinte. Toutefois, ce phénomène de libération, de triomphe des indépendances nationales, de recul du colonialisme, ne se présente pas d'une manière unique. Chaque ancienne colonie a une façon déterminée d'accéder à l'indépendance. Chaque nouvel Etat souverain se trouve pratiquement dans l'obligation de maintenir avec l'ancien oppresseur des relations définies et préférentielles.

26 El Moudjahid, № 27 du 22 juillet 1958. 
Les partis qui mènent la lutte contre l'oppression colonialiste, à une certaine phase du combat, décident tactiquement d'accepter une parcelle d'indépendance avec la ferme intention de relancer le peuple dans le cadre de la stratégie fondamentale de l'évacuation totale du territoire et de la prise en mains effective de toutes les ressources nationales. Ce style classique, et plusieurs fois incarné, est aujourd'hui connu. Par contre, il existe toute une dialectique opposée qui, semble$t-i l$, n'a pas retenu suffisamment l'attention.

[142]

\section{Première hypothèque: «Les Droits » de l'ancien occupant}

Il y a quelques décades, les gouvernants colonialistes pouvaient indéfiniment exposer les préoccupations hautement civilisatrices de leurs pays. Les concessions, les expropriations, l'exploitation des travailleurs, la grande misère des peuples, étaient traditionnellement escamotées et niées. Par la suite, au moment de se retirer de ce territoire, les colonialistes furent mis dans l'obligation de jeter leurs masques. Dans les négociations sur l'indépendance, il était d'abord question des intérêts économiques: banques, zone monétaire, permis de recherches, concessions d'exploitation, inviolabilité des propriétés volées aux paysans lors de la conquête, etc... D'œuvre civilisatrice, évangélique on culturelle, il ne fut plus question. L'heure était aux choses sérieuses et non aux balivernes. De telles attitudes devaient éclairer la conscience des hommes en lutte dans d'autres régions du monde.

Les véritables droits de l'occupant furent alors parfaitement identifiés. Minorité issue de la métropole, missions universitaires, assistance technique, amitié affirmée et réaffimée, sont passées au second plan. De toute évidence, l'important devenait les droits réels que l'occupant entendait arracher au peuple, comme prix d'un morceau d'indépendance.

L'acceptation d'une souveraineté nominale et le refus absolu d'une indépendance réelle, telle est la réaction-type des nations colonialistes à l'égard de leurs anciennes colonies. Le néocolonialisme est imprégné 
par quelques idées qui, à la fois, font sa force en préparant sa nécessaire décadence.

Au cours de la lutte de libération, les choses ne sont pas claires dans la conscience du peuple qui se bat. $A$ la fois refus de l'inexistence politique, de la misère, de l'analphabétisme, du complexe d'infériorité distillé savamment par l'oppression, son combat est pendant longtemps indifférencié. Le néo-colonialisme va mettre à profit cette indétermination. Armé d'une bienveillance révolutionnaire et spectaculaire, il va tout reconnaître à l'ancienne colonie. Mais, ce faisant, il lui arrache une dépendance économique qui devient programme d'aide et d'assistance.

Nous avons vu que la plupart du temps cette tentative [143] triomphe. L'originalité de cette phase est qu'elle est nécessairement courte. C'est qu'il faut peu de temps au peuple pour qu'il s'aperçoive que rien de fondamental n'a été changé. Passées les heures d'effusion et d'enthousiasme devant le spectacle du drapeau national flottant dans le vent, le peuple retrouve la dimension première de son exigence : du pain, des vêtements, un abri.

Le néo-colonialisme, parce qu'il se propose de rendre justice à la dignité humaine en général, s'adresse essentiellement à la bourgeoisie et aux intellectuels du pays colonial.

Aujourd'hui, les peuples ne se sentent plus le ventre en paix quand le pays colonial a reconnu la valeur de ses élites. Les peuples veulent que cela change réellement et tout de suite. C'est ainsi que la lutte reprend avec une violence irréfragable.

Dans cette deuxième phase, l'occupant se hérisse et lance toutes ses forces. Ce qui fut arraché par les bombardements est reconverti en résultats de libres négociations. L'ancien occupant intervient, fort de ses devoirs, et installe de nouveau sa guerre dans un pays indépendant.

Toutes les anciennes colonies, de l'Indonésie à l'Egypte en passant par Panama, qui ont voulu dénoncer les accords arrachés par la force, se sont trouvées dans l'obligation de soutenir une nouvelle guerre et quelquefois de voir leur souveraineté de nouveau atteinte et amputée.

Les fameux droits de l'occupant, le chantage au passé de vie commune, la persistance d'un pacte colonial rajeuni, sont les bases permanentes d'une attaque menée contre la souveraineté nationale. 


\section{Deuxième hypothèque: les zones d'influence}

Le souci de maintenir l'ancienne colonie dans le carcan de l'oppression économique n'est évidemment pas du sadisme. Ce n'est pas par méchanceté ou mauvais vouloir qu'une telle attitude est adoptée. C'est que la gestion par les peuples coloniaux de leurs richesses nationales compromet l'équilibre économique de l'ancien occupant. La reconversion de l'économie coloniale, les industries de transformation de matières premières provenant des territoires sous-développés, la disparition [144] du pacte colonial, la compétition avec les capitaux étrangers, constituent un danger de mort pour l'impérialisme.

Pour les nations comme l'Angleterre et la France interfère l'importante question des zones d'influence. Unanimes dans leur décision de briser la revendication nationale des peuples coloniaux, ces pays mènent une lutte gigantesque pour l'accaparement des marchés mondiaux. Les batailles économiques entre la France, l'Angleterre et les Etats-Unis, au Moyen-Orient, en Extrême-Orient et maintenant en Afrique, donnent la mesure de la voracité et de la bestialité impérialistes. Et il n'est pas exagéré de dire que ces batailles sont la cause directe des stratégies qui, encore aujourd'hui, secouent les Etats nouvellement indépendants. Dans les circonstances exceptionnelles, les zones d'influence de la livre sterling, du dollar ou du franc, se convertissent et deviennent, par un tour de passe-passe, le monde occidental, Aujourd'hui, au Liban et en Irak, à en croire M. Malraux, c'est l'homo occidentalis qui se trouve en danger.

Le pétrole irakien a levé toutes les interdictions et actualisé les véritables problèmes. On a en mémoire les interventions violentes des forces armées américaines dans l'archipel des Antilles ou en Amérique latine, chaque fois que les dictatures appuyées par la politique américaine se sont trouvées en danger. Les Marines qui déferlent aujourd'hui sur Beyrouth sont les frères de ceux qui, épisodiquement, vont rétablir « l'ordre » en Haïti, à Costa-Rica, à Panama. C'est que les Etats-Unis estiment que les deux Amériques constituent un monde régi par la doctrine de Monrö̈, dont l'application est confiée aux forces 
américaines. L'article unique de cette doctrine stipule que l'Amérique appartient aux Américains, c'est-à-dire au Département d'État.

Ses débouchés s'étant révélés insuffisants, l'Amérique devait fatalement se tourner vers d'autres régions, en 1'occurrence l'ExtrêmeOrient, le Moyen-Orient et l'Afrique. Compétition de rapaces: ses créations sont: Doctrine Eisenhower contre l'Angleterre au MoyenOrient ; soutien à Ngo Din Diem contre la France en Indochine: Commission d'aide économique en Afrique annoncée par le voyage présidentiel de M. Nixon, contre la France, l'Angleterre et la Belgique.

Chaque lutte de libération nationale doit tenir compte des zones d'influence.

\section{La guerre froide}

Cette stratégie compétitive des nations occidentales entre, par ailleurs, dans le cadre plus vaste de la politique des deux blocs qui fait peser sur le monde, depuis dix ans, une menace précise de désintégration atomique. Et ça n'est certes pas un hasard si, derrière chaque revendication nationale d'un peuple colonial, on découvre, de façon presque stéréotypée, la main ou l'œil de Moscou. C'est que toute difficulté faite à la suprématie de l'Occident dans une parcelle du monde est une atteinte concrète à sa puissance économique, à l'étendue de ses bases stratégiques militaires, à une limitation de son potentiel.

Chaque contestation des droits de l'Occident sur un pays colonial est à la fois vécue comme affaiblissement du monde occidental et renforcement du monde communiste.

Aujourd'hui, une île comme Chypre, qui n'a presque pas de ressources propres et qui a une population d'à peine un demi-million d'hommes fait l'objet de rivalités violentes. Et il n'est pas jusqu'à l'O.T.A.N., cette organisation chargée de stopper l'invasion soviétique, qui ne soit mise en danger par les problèmes qui se posent autour de lîle de Chypre. 


\section{Le troisième bloc}

La prise de position de quelques pays nouvellement indépendants, décidés à demeurer en dehors de la politique des blocs, a introduit une dimension originale dans l'équilibre des forces dans le monde. Politique dite de neutralisme positif, de non-dépendance, de non-engagement, troisième force, les pays sous-développés qui se réveillent d'un long sommeil d'esclavage et d'oppression, ont estimé de leur devoir de demeurer en dehors de toute préoccupation belliciste, afin de se consacrer à l'essor économique, au recul de la faim, à la promotion de I'homme.

Et, en vérité, ce que les Occidentaux n'ont pas compris, c'est qu'il se crée aujourd'hui, un nouvel humanisme, une nouvelle théorie de l'homme qui a sa racine dans l'homme et qui ne veut autre chose que le triomphe inégalé de cet homme. Il est facile de traiter le Président Nehru d'indécis parce qu'il refuse de s'atteler à l'impérialisme occidental, les Présidents [146] Nasser ou Soekarno de violents quand ils nationalisent leurs compagnies ou revendiquent les Parcelles de leurs territoires encore sous domination étrangère. Ce que l'on ne voit pas, c'est que les 350 millions d'Hindous, qui ont connu la faim de l'impérialisme anglais, réclament actuellement du pain, la paix et le bien-être. C'est que les fellahs égyptiens ou les boys indonésiens, qui permettaient aux écrivains occidentaux de faire ouvre exotique, exigent de prendre en mains leur destinée et refusent de jouer le rôle de panorama inerte auquel ils étaient dévolus.

\section{Le prestige de l'Occident}

Et nous touchons ici à un problème psychologique qui n'est certes pas fondamental, mais qui entre dans la constitution de la dialectique qui se développe actuellement. Système économique de référence, donc d'oppression, l'Occident se prévaut aussi de sa supériorité humaniste. «Le modèle » occidental se trouve atteint dans son essence et dans sa finalité. Les Jaunes les Arabes et les Nègres, aujourd'hui, veu- 
lent dire leurs projets, veulent affirmer leurs valeurs, veulent définir leurs relations avec le monde. La négation du béni-oui-ouisme politique est liée au refus du béni-oui-ouisme économique et du béni-oui-ouisme culturel. Il n'est plus vrai que la promotion des valeurs passe par le tamis de l'Occident. Il n'est pas vrai qu'il nous faille constamment être à la remorque, suivre, dépendre de qui que ce soit. Tous les pays coloniaux qui mènent la lutte aujourd'hui, doivent savoir que l'indépendance politique qu'ils arracheront à l'ennemi contre le maintien d'une dépendance économique n'est qu'un leurre, que la deuxième phase de libération totale est nécessaire parce que exigée par les masses populaires, que cette deuxième phase, parce que capitale, doit être dure et menée avec aridité, qu'enfin, à ce stade, il faudra tenir compte de la stratégie mondiale des blocs, car l'Occident est à la fois confronté avec un double problème: le danger communiste et l'apparition d'un troisième bloc neutraliste, représenté essentiellement par les pays sousdéveloppés.

Le devenir de tout homme entretient aujourd'hui des rapports d'étroite dépendance avec le reste de l'univers. C'est pourquoi les peuples coloniaux doivent redoubler de vigilance [147] et de vigueur. L'apparition d'un nouvel humanisme est à ce prix. Les loups ne doivent plus trouver de brebis isolée. Il faut que l'impérialisme soit stoppé dans toutes ses tentatives de renforcement. Les peuples le veulent, le processus historique l'exige. 


\author{
Pour la révolution africaine \\ Écrits politiques
}

Quatrième partie. Vers la libération de l'Afrique.

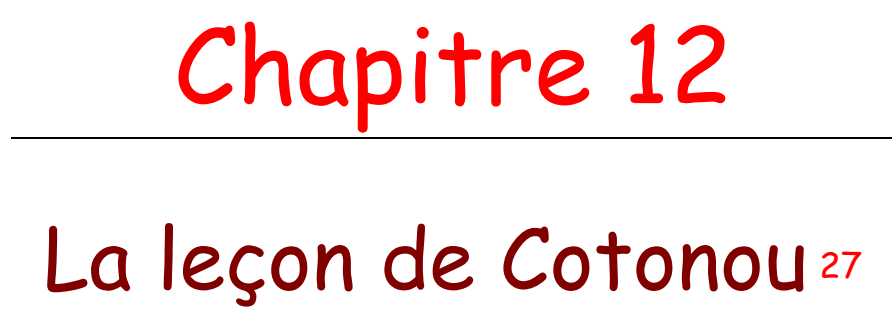

\title{
Retour à la table des matières
}

Le Congrès adopte le mot d'ordre d'indépendance immédiate et décide de prendre toutes les mesures nécessaires pour mobiliser les masses africaines autour de ce mot d'ordre et de traduire dans les faits cette volonté d'indépendance.

C'est par ces termes que prend fin la déclaration de guerre qui vient d'être adressée, de Cotonou, au gouvernement français du Général de Gaulle par les peuples d'Afrique Noire.

Déjà, à Bamako, en septembre 1957, le dispositif de sécurité mis en place par la Loi-Cadre avait sauté. Le traître Houphouët-Boigny et ses complices avaient dû reculer devant la revendication nationale africaine. Tous les spécialistes en léthargie coloniale s'étaient alors rendus sur place afin d'y voir consacrée leur victoire.

Or, à Bamako, les Africains ont rejeté la Loi-Cadre, rejeté l'irresponsabilité, à laquelle le colonialisme les condamnait.

27 El Moudjahid, № 28 du 22 août 1958. 
À Bamako, le verrou de sûreté en Afrique Noire était pulvérisé. Un processus se mettait en marche. Cotonou est l'avant-première de la grande lutte de libération à la suite de laquelle plus de 30 millions d'Africains accèderont à l'indépendance.

On se souvient de l'euphorie qui régna dans les milieux politiques français après le vote de la Loi-Cadre. Pour une fois, affirmait-on, la France prend l'initiative et, selon «sa plus essentielle tradition», devance la revendication des peuples. Or, précisément, l'observateur lucide des choses d'Afrique [150] Noire savait parfaitement que la loi Defferre était infiniment en-deçà des aspirations des masses africaines.

Les ouvriers africains, les étudiants africains sont depuis plusieurs années contenus, retenus par les parlementaires africains.

En Afrique Noire depuis 1947 le colonialisme français doit sa quiétude à la trahison inqualifiable de certaines élites africaines.

Depuis 1947, de façon diffuse et de façon beaucoup plus concertée depuis 1953, les syndicalistes africains développent leur action selon une perspective délibérément nationale.

Eloignées de tout corporatisme, s'inscrivant dans la double optique de l'africanisation des cadres et de la nationalisation, donc de l'indépendance, les actions syndicales ont imprimé à la lutte contre le colonialisme français un style absolument nouveau.

L'U.G.T.A.N., qui étend ses filets sur l'ensemble du pays asphyxie progressivement la bête colonialiste. Face aux ouvriers qui réclament la nationalisation et la socialisation des entreprises et des propriétés, le colonialisme, atterré, s'apprête à mobiliser de nouveaux mercenaires pour défendre, en Afrique Noire, la civilisation occidentale.

De son côté, la Fédération des Etudiants d'Afrique Noire a entrepris depuis plusieurs années un travail de propagande en profondeur, de démystification actuelle, de mise en place de mots d'ordre clairs. Aussi, la police du traître Houphouët-Boigny, s'est-elle acharnée sur les responsables étudiants. La police des villes universitaires en France, à la demande du ministre Houphouët-Boigny, a persécuté, arrêté, torturé des étudiants d'Afrique Noire.

Suppression de bourses, chantages, saisies et interdiction du journal de la Fédération des Etudiants, autant d'opérations réalisées sous 
l'autorité de Houphouët-Boigny. De plus, en Afrique Noire, les étudiants, opposés à la politique de trahison de certains dirigeants du R.D.A., ne sont pas employés comme fonctionnaires par les ridicules conseils de gouvernements; ils sont matés, licenciés, expulsés du territoire. En dépit de ces manœuvres policières, malgré ces mesures d'intimidations, les étudiants d'Afrique Noire, à Cotonou, par la voix de leur président, ont affirmé leur ferme décision de passer à [151] l'action directe et d'arracher l'indépendance nationale par les armes.

M. Defferre, qui devait attacher son nom à cette parodie de politique libérale, a eu, depuis, le temps de méditer sur « l'ingratitude des Africains ». Avec la Loi-Cadre, le colonialisme français espérait avoir contracté un engagement d'une bonne vingtaine d'années contre le virus nationaliste. La Loi-Cadre officialisait la division du continent africain, éparpillait ça et là des conseils de gouvernement, créait des zones économiques compétitives. La centralisation, l'unité territoriale, la constitution de la nation, l'intégration économique du pays faisaient place à la hideuse balkanisation de l'A frique Noire.

Le tort de M. Houphouët-Boigny et de ses complices est de n'avoir pas suffisamment tenu compte du boutoir algérien qui, depuis quatre ans, ébranle dans ses fondements l'empire français.

Pour s'être accroché au-delà du bon sens en Algérie, le colonialisme français devra y perdre la vie. Avec la fin de la guerre d'Algérie, avec la victoire des forces armées de l'A.L.N. et l'indépendance de l'Algérie, c'est tout le système impérial français qui s'écroulera.

La résolution du Congrès de Cotonou n'est pas importante pour la seule raison qu'elle pose comme objectif l'indépendance immédiate. Les termes mêmes dans lesquels cette indépendance est exigée, le contenu social, la netteté avec laquelle sont utilisées les notions de socialisme, de collectivisation, de communauté progressiste, indiquent que les Africains ne visent pas une indépendance formelle.

À Cotonou, nous n'assistons pas à l'évolution des esprits africains mais bien à une mutation essentielle qui débouche sur la prise en mains effective du pays par le peuple.

Depuis Cotonou, le colonialisme français est atterré et sans voix.

Or, depuis quatre ans, parce que la Révolution algérienne existe en Afrique, la revendication nationale des autres peuples africains 
s'inspire largement du mouvement même de notre Révolution. Depuis quatre ans, c'est l'existence même du colonialisme en Afrique qui est mise en morceaux par le peuple algérien et il est banal de reconnaître aujourd'hui qu'un certain [152] nombre de pays indépendants en 1958 ne le seraient certainement pas si le colonialisme français, entre autres, n'avait eu à faire face aux innombrables coups que lui portait le peuple algérien.

Guy Mollet, Bourgès-Maunoury, Gaillard, Pflimlin, le de Gaulle sauveur de la France, pré-fasciste avant d'être totalement tel, sont les accidents historiques divers qui ont été suscités en France par la rencontre armée de la volonté nationale du peuple algérien et de la volonté d'oppression colonialiste des gouvernements français.

Cette courbe de la vie politique française évoluant, depuis 1954, de la social-démocratie traîtresse à ses plus élémentaires principes, jusqu'à la dictature fasciste et militaire, tel est le négatif de l'héroïsme révolutionnaire du peuple algérien.

C'est pourquoi, désormais, il ne saurait y avoir en Afrique d'hésitation de la part des masses nationales. D'emblée et d'un violent coup d'épaule, les voici au niveau de la revendication immédiate et totale.

Le colonialisme français cherche une réponse colonialiste aux aspirations nationales africaines exprimées à Cotonou. Certains observateurs estiment que de Gaulle trouvera un moyen terme entre Loi-Cadre et indépendance.

Le F.L.N., après une analyse des faits et compte tenu des difficultés insurmontables qu'il crée à la France en Algérie, estime que les peuples d'Afrique Noire sous domination française ne doivent pas reculer mais au contraire montrer avec fermeté et brutalité que le moment des solutions confuses est irrémédiablement révolu.

La France est acculée, il faut davantage l'acculer, lui couper toutes les issues, l'asphyxier sans pitié, tuer en elle toute velléité de domination. En 1958, la France est incapable matériellement et humainement, économiquement et politiquement, d'entreprendre une guerre en Afrique Noire.

C'est pourquoi les peuples africains doivent aller de l'avant, accentuer leur pression et exiger, dans l'instant, leur indépendance. Les 
masses africaines et les élites africaines doivent dès maintenant prendre leurs dispositions pour passer à l'action directe, prendre les armes, semer la panique dans les rangs colonialistes.

Le F.L.N. et I'A.L.N. sont prêt, quant à eux, à aider les peuples africaine dans leur lutte de libération. Il ne sera pas [153] dit que l'impérialisme français après son départ d'Algérie pourra encore se maintenir en Afrique. Le mot d'ordre aujourd'hui doit être:

«Africains, Africaines, aux armes!

Mort au colonialisme français!» 
[155]

\author{
Pour la révolution africaine \\ Écrits politiques
}

Quatrième partie. Vers la libération de l'Afrique.

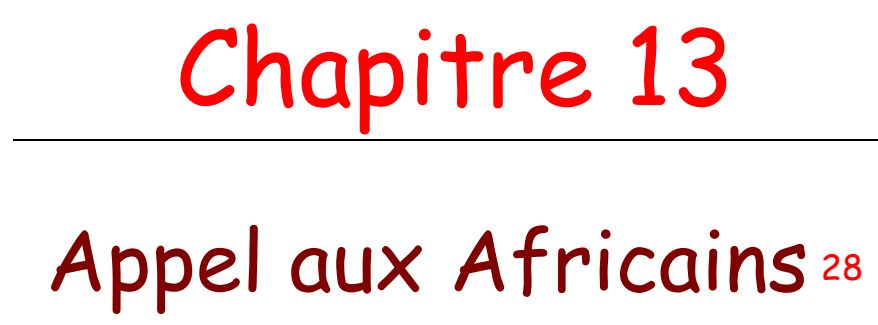

\title{
Retour à la table des matières
}

Lorsque le général de Gaulle, au cours de son voyage outre-mer, dut préciser, sous la poussée des masses africaines, le sens du référendum - pour les territoires sous domination coloniale - la gauche française et l'opinion internationale crurent voir dans cette déclaration la première manifestation de ce qu'il est convenu d'appeler le libéralisme du chef du gouvernement français.

Les premières heures de surprise passées, il fallut bien revenir aux textes, aux réalités, donc aux possibilités concrètes laissées aux hommes d'Afrique Noire.

En réalité, grâce à l'opération référendum, le général de Gaulle engage toutes les «possessions françaises » dans un processus indéfini de domestication librement consentie. D'abord, comment se présente le référendum face aux aspirations nationales africaines? Le colonialisme français oppose son armée, sa police, ses traditionnels beni-oui- 
oui et s'appuie sur des assemblées locales largement compromises et démonétisées.

Si dans certains Etats le Conseil de Gouvernement a adopté une attitude de revendication nationale, le colonialisme français, dans la majorité des cas, s'est assuré le soutien d'hommes politiques africains.

Les Africains qui demandent à leurs compatriotes de plébisciter de Gaulle et «la communauté franco-africaine " manifestent [156] une grande incompréhension des problèmes de la décolonisation et une ignorance criminelle des aspirations nationales des peuples africains.

Participer au vote, exprimer des suffrages sur des questions strictement françaises, donne corps à cette « union française » transformée par nécessité en «communauté franco-africaine», aliène la personnalité africaine et, comme le dit la constitution, installe une nationalité unique.

Participer au vote, c'est tacitement se reconnaître membre d'une même famille, d'une même nation ayant des problèmes communs alors qu'en réalité chaque Africain qui votera à l'occasion du référendum, enchaînera un peu plus son peuple et son pays au colonialisme français.

La présence massive de forces militaires et policières françaises en Afrique Noire, l'agitation des hommes politiques compromis, leurs déclarations, leurs invitations empressées à leur peuple de voter oui, les traditions de truquage des élections, ne laissent aucun doute sur les résultats du référendum en Afrique Noire.

Dans un certain nombre d'états, la constitution française sera plébiscitée à une très forte majorité.

Or, dès le $1^{\text {er }}$ Octobre, quatre véritables problèmes vont se poser aux Français et aux Africains. Les Etats africains délègueront-ils des députés à l'Assemblée Nationale française? La représentation de 30 millions d'Africains sera-t-elle proportionnelle? Les Africains aurontils le droit de discuter de leur budget, auront-ils la possibilité de diriger les investissements concernant leurs territoires respectifs? La France entend-elle, malgré l'opposition des Africains, donner naissance à cette « Eurafrique » qui doit consacrer le morcellement de l'Afrique en aires d'influences européennes et pour le seul bien de ces économies européennes? La France entend-elle maintenir les Etats africains dans le cadre de l'O.T.A.N. ? Les Etats africains, à l'ère de Bandoeng, 
veulent exister à l'échelon international et revendiquent leur place à I'O.N.U. Comment la France espère-t-elle concilier à la fois le maintien du pacte colonial et l'existence nationale des Etats africains?

[157]

Toutes ces questions se poseront au lendemain du référendum, lorsque les Africains et les Africaines mesureront la profondeur de la mystification.

Encore une fois, le colonialisme français à long terme joue perdant. De Gaulle, en Afrique, n'aura rien apporté de décisif. Les mêmes problèmes subsisteront, les mêmes exigences, la même revendication nationale.

Le colonialisme français s'opposera, avec la même mauvaise foi, les mêmes méthodes, à ces revendications nationales. La lutte continuera donc avec cette différence toutefois que la phase parlementaire semble définitivement écartée et que, en Afrique Noire, il est de plus en plus question d'engager la lutte armée pour la libération du territoire national.

Une fois de plus, le colonialisme français se ferme toutes les portes. Au lieu de convier les représentants authentiques des peuples qu'il domine à une discussion constructive qui débouche sur la fin du pacte colonial et la reconnaissance des souverainetés nationales particulières, il perpétue le cycle du mensonge, de la terreur, de la guerre, rendant par là extrêmement difficile la réconciliation des peuples. 


\section{Pour la révolution africaine \\ Écrits politiques}

Quatrième partie. Vers la libération de l'Afrique.

\section{Chapitre 14 \\ Lendemains d'un plébiscite en Afrique 29}

\section{Retour à la table des matières}

Classiquement, le succès du plébiscite assure aux autorités responsables du coup d'Etat une période plus ou moins longue de jouissance du pouvoir. L'exercice de l'autorité rendu possible par le plébiscite pour être valable et pour récompenser les auteurs du putsch, doit pouvoir leur permettre d'en tirer le maximum d'avantages dans le maximum de temps et dans une relative quiétude.

L'action conjuguée des colonialistes français, des fascistes avec leur avant-garde prétorienne à Alger, et des forces réactionnaires en France, a pu imposer l'arbitrage du général De Gaulle.

29 El Moudjahid, N 30 du 10 octobre 1958. 


\section{La préparation du complot}

Les forces fascistes de ce complot se proposaient d'utiliser le nom du général De Gaulle pour mettre sur pied des organisations capables de prendre le pouvoir effectif, quitte pour elles à se débarrasser par la suite du Général-Président.

Pendant ces trois derniers-mois, en France et en Algérie principalement, on a assisté, autour du noyau de base des factieux du 13 mai, à une cristallisation de tous les courants racistes, ultra-chauvins, fascistes, qui existent chez les Français. On a assisté pareillement à la colonisation de l'appareil de l'Etat par les membres de ces milices.

[160]

Les mesures, classiques elles aussi, qui succèdent toujours à un plébiscite furent intempestivement annoncées : mise à la raison des partisans de l'abandon et surtout mise hors-la-loi des groupements d'opposition.

Les forces colonialistes, qui ont cautionné de leur autorité et ont appuyé totalement l'expérience fasciste, étaient habitées par la terreur d'un processus quelconque de décolonisation. Après les expériences de la Tunisie et du Maroc et celle, beaucoup plus traumatisante de l'Indochine, les milieux colonialistes avaient fait le serment de ne plus tolérer de nouveaux abandons. Aussi ces milieux colonialistes ont-ils rejoint avec enthousiasme les rangs fascistes aux cris de: "Algérie française », « Halte à l'abandon ».

Dans le même temps, les «bradeurs d'empire», type MendèsFrance, Edgar Faure, se voyaient accusés de trahison.

Les milieux colonialistes ont rejoint le général De Gaulle parce que, à leurs yeux, il était capable de garder l'Algérie à la France, de maintenir intact l'Empire français et peut-être si l'occasion s'en présentait, de reconquérir les territoires perdus.

Les réactionnaires, pour leur part, qui ont répondu à l'appel fasciste étaient surtout animés du désir des forces gratuites payées par l'Etat et dont les intérêts allaient dans le même sens que les leurs pour 
consolider leur autorité en France et briser l'action et les espoirs de la classe ouvrière française.

\section{La victoire...}

Au lendemain du 28 septembre, les responsables du coup d'Etat pouvaient donc se déclarer satisfaits. Dans les territoires « d'Outremer » $98 \%$ de coloniaux avaient répondu « Oui » au général De Gaulle et à la France. Sur le territoire français, $80 \%$ de nationaux ayant compris leurs intérêts et s'étant détachés de la tutelle des « partis de l'Etranger » avaient adhéré massivement à la charte proposée par De Gaulle.

Une semaine après le référendum, une semaine après ce succès colossal, massif, miraculeux, l'entreprise fasciste commence à vaciller. La guerre d'Algérie, autour de laquelle s'est organisée le référendum qui a permis par son exploitation le succès grandiose signalé par la presse, limite par son existence, son caractère et sa durée, la valeur de ce plébiscite. Au lendemain [161] du 28 septembre, embarrassés par leur $98 \%$ de voix, le général De Gaulle et ses suppôts ne savent plus où donner de la tête. C'est que, jour après jour, des données nouvelles apparaissent qui nuancent la portée des « oui ».

\section{... Et la déconfiture}

En Afrique occidentale, M. Mokhtar Ould Daddah, président du Conseil de Mauritanie, territoire qui a voté oui à $93 \%$, déclarait le $1^{\text {er }}$ octobre:

«Je pense que l'actuelle Assemblée Territoriale devra démissionner pour permettre l'élection en janvier d'une nouvelle Assemblée, à laquelle le Conseil de Gouvernement actuel remettra sa démission. Le nouveau gouvernement et la nouvelle Assemblée opteraient pour le statut d'Etat membre de la commu- 
nauté et élaboreraient la constitution locale qui sera soumise au peuple mauritanien par voie de référendum », et $M$. Ould Daddah ajoutait: "Nous sortirons alors, le moment venu, de la communauté des peuples libres, prévue au Titre 12, sans rompre pour autant nos liens avec la France et les Etats de la Communauté et nous concluerons avec elle les accords d'association prévus au Titre $13 »$.

Au Dahomey où le pourcentage de voix affleure $98 \%$, M. Apithy, président du Conseil de gouvernement vient d'annoncer la nécessité pour les leaders politiques d'Afrique Noire de se consulter pour adopter une attitude commune en face de la France. Précisant quant à lui, le sens du oui dahoméen. M. Apithy ajoutait: «Par le libre choix de ses masses et la libre volonté de ses élus, naîtra demain dans la communauté, l'État dahoméen. Pleinement autonome, cet Etat pourrait préparer dans la paix et les amitiés conservées avec les autres peuples africains aussi bien qu'avec, la métropole, son accession au développement économique et social qui lui permettra un jour de devenir un Etat associé indépendant».

Au Sénégal où l'adhésion au « oui » a été de $97 \%$, l'opposition syndicaliste et les formations de jeunesse continuent à faire pression sur MM. Senghor et Lamine Gueye. Déjà à Cotonou, M. Senghor qui semble ne pas vouloir se libérer de la confusion qui règne dans son esprit avait été obligé de se rallier à l'indépendance de l'Afrique Noire. Il a pu, réalisant une alliance tactique avec son adversaire Lamine Gueye, tromper les masses africaines en leur faisant voter oui. Or [162] Senghor au lendemain du succès du plébiscite se trouve acculé à donner au vote du Sénégal une signification précise. C'est un oui - dit-il - à l'indépendance africaine dans l'unité retrouvée.

Il est évident que Senghor tentera encore d'abuser les masses sénégalaises mais ses possibilités d'action se voient les unes après les autres limitées. La récente déclaration de Mamadou Dia, président du Conseil de gouvernement du Sénégal est significative: «Nous avons, dit-il, essentiellement le souci de préparer notre indépendance ».

Au Niger, le gouvernement a pu faire échec à la politique nationaliste de Djibo Bakary. On s'aperçoit que le Niger est le premier territoire africain après la Guinée qui possède le plus de suffrages négatifs. 
Avec l'aide des colonialistes et des administrateurs, le gouvernement a pu assurer le succès de la consultation électorale du 28 septembre, mais il est clair que les masses nigériennes vont exercer dans les semaines qui viennent une action décisive pour exiger que soient prises en considération leurs revendications nationales. De toute évidence, le moins que puissent faire les autres territoires de l'A frique occidentale y compris la fidèle Côte d'Ivoire, c'est de choisir le statut d'Etat autonome associé à la France.

M. Houphouët-Boigny essaiera peut-être de défendre sa théorie du fédéralisme intégral mais il est douteux qu'il puisse parvenir à ses fins. L'Afrique occidentale malgré De Gaulle, malgré le 13 mai, malgré le référendum, va se fédérer en Etats autonomes pour, dans un second temps, étudier avec les représentants français, les modalités de coopération avec l'ancienne métropole.

En Afrique Equatoriale, les choses sont beaucoup plus claires. Au Gabon les milieux colonialistes ont été secoués par l'importance des «non » : plusieurs dizaines de milliers.

La force politique de l'opposition est telle au Gabon que M. Léon $\mathrm{M}^{\prime} \mathrm{Ba}$, président du Conseil du gouvernement, quittant Libreville le 3 octobre afin d'engager des discussions à Paris avec le gouvernement français, faisait part de sa décision de créer un Etat gabonais. Mais ce qui est beaucoup plus important c'est l'annonce, il y a quelques jours, de la constitution d'une mission du grand conseil de l'Afrique équatoriale, chargée d'étudier la création d'un Etat unique en Afrique centrale. M. Rivierez, président de l'Assemblée territoriale de l'OubanguiChari a commencé des consultations avec les [163] représentants du Tchad, du Moyen-Congo et du Gabon afin de mettre sur pied l'Etat, la nationalité et de préparer les rapports entre les autres territoires d'Afrique et de la France. La vieille loi-cadre qui avait pour fonction de morceler l'Afrique rejoint dans les musées de l'Histoire, les autres tentatives faites par le colonialisme pour se maintenir.

À Madagascar, où le béni-ouiste Tsiranana n'a pas craint de commenter le « oui » malgache comme un « non » à Moscou, le colonialisme n'est pas plus heureux. M. Tsiranana, le Sid-Cara de Madagascar, dans une déclaration faite au lendemain du référendum, admet que les Malgaches vont enfin voir réaliser la République malgache. 
Aux Antilles, d'aucuns se sont étonnés du pourcentage des «oui » obtenus grâce à la position de M. Aimé Césaire. L'indépendance des Antilles françaises est-elle possible aujourd'hui ? Telle est la question devant laquelle s'est trouvé Césaire. Il pouvait répondre par l'affirmative, choisir l'indépendance des Antilles et solliciter l'entrée dans la fédération caraïbe. A-t-il estimé précisément que cette fédération en train de naître n'était pas suffisamment structurée et dépendait encore trop étroitement de la couronne britannique? Ce qui est certain, c'est qu'aux Antilles comme ailleurs, aucun leader ne peut s'illusionner sur la valeur de sa personne ni l'amour que lui porteraient les masses dès lors que se trouve engagée la volonté d'indépendance nationale de leur pays.

\section{Le cas de la Guinée}

Pour amorcer la libération de l'Afrique Noire, il fallait une chose: qu'un territoire au moins dise non à la Constitution du général De Gaulle. La Guinée derrière M. Sekou Touré est entrée dans l'indépendance.

L'existence d'une Guinée indépendante déséquilibre profondément et irréversiblement le régime colonial français en Afrique Noire. Possédant des frontières communes avec le Sénégal, le Soudan et la Côte d'Ivoire, la Guinée va cristalliser autour d'elle toutes les potentialités nationalistes existant en Afrique Noire. On a prétendu que les autres leaders politiques africains avaient rejeté le «non» par crainte de représailles économiques du gouvernement français. Nul n'ignore cependant la fragilité de tels arguments. Les hommes politiques [164] comme Houphouët-Boigny et l'abbé Fulbert Youlou qui ont milité en faveur des « oui » sont en réalité des contre-révolutionnaires, des ennemis de l'indépendance nationale de l'A frique.

Aujourd'hui la République guinéenne, reconnue par un nombre croissant de nations, soutenue par les patriotes africains de tous les territoires, renforce son autorité et écarte de son chemin toute peur et toute crainte. Les autres dirigeants d'Afrique Noire, les mêmes qui annonçaient des catastrophes en Guinée, se concertent et découvrent que le carcan du colonialisme en Afrique Noire a sauté depuis longtemps. Le tête-à-tête obligatoire avec le seul colon, la violence avec 
laquelle la domination française était vécue s'émiettent. L'Union Soviétique vient de reconnaître la République guinéenne et les Etats africains à la demande de la Tunisie viennent de proposer à M. Sekou Touré l'envoi d'une mission chargée d'étudier les besoins matériels et techniques de la jeune République.

Illustrant cette solidarité africaine forgée dans la lutte contre le colonialisme, le Gouvernement Provisoire de la République guinéenne concrétise les lignes directrices qui inspirent notre action et préfigure les liens d'étroite collaboration qui doivent exister entre les nouveaux Etats indépendants d'Afrique.

Les hommes d'Afrique Noire, parce que la Guinée indépendante existe, pourront très rapidement comparer leur sort à celui de leurs frères d'hier, esclavagisés par le colonialisme français.

M. Houphouët-Boigny n'a eu de cesse qu'il n'ait convaincu les peuples africains que le statut d'indigène était le plus enviable. Que sans la protection de la mère-patrie française, les nègres d'Afrique Noire seraient abandonnés.

C'est de la Guinée, tête de pont de la liberté, que partiront toutes les vagues qui anéantiront la domination française en Afrique Noire.

\section{Le référendum en France}

Les observateurs étrangers ont généralement été impressionnés par le pourcentage de votes positifs obtenus par le général De Gaulle. Les prévisions les plus optimistes faisaient [165] état de 65 à $70 \%$. Or c'est à $80 \%$ que le référendum a été accueilli en France. On a rapidement diagnostiqué une désaffection des Français à l'égard des formations politiques de gauche.

Une analyse du scrutin permet toutefois d'aboutir à d'autres conclusions. Pour 17.600 .000 « oui », on trouve 4.600.000 «non». Treize millions de voix d'écart donnent ainsi la mesure du prestige présidentiel. Les communistes accusent un recul très net évalué au tiers. De plus, les leaders politiques de gauche, Badiou, MendèsFrance, Bourgès-Maunoury qui ont milité pour le refus de la Constitution, seraient les grands vaincus de cette confrontation électorale. Il 
semble donc qu'on ait assisté avec le référendum à une mise en hibernation des perspectives démocratiques en France.

Or, les déclarations d'hommes politiques comme M. Defferre, altèrent l'homogénéité des résultats. Des millions de Français et de Françaises ont voté oui pour que prenne fin la guerre d'Algérie. D'autres pour que l'empire demeure intact. On a vu que le référendum dans les territoires coloniaux amorçait irréversiblement la libération des peuples opprimés et la dernière déclaration à Constantine du général De Gaulle est une authentique relance de la guerre. Après avoir promis l'aman aux « rebelles», le président du Conseil français, dans la bonne tradition de ces collègues de la défunte quatrième, annonce le progrès social et économique.

Ayant pris le pouvoir pour faire la paix en Algérie, De Gaulle provoque l'extension du conflit en France. Depuis le 24 août, les bases économiques et stratégiques françaises sont sabotées par les groupes d'action du F.L.N. Guerre en Algérie, guerre en France, le colonialisme, son corps expéditionnaire et ses bases de soutien se trouvent sous les coups de la Révolution algérienne. La paix en Algérie, la paix en France dépendent aujourd'hui de la reconnaissance par la France de l'indépendance de l'Algérie.

\section{De Gaulle mis en échec par le peuple algérien}

On peut légitimement se demander les causes de cette détérioration de la situation en Afrique Noire. Comment un succès aussi colossal que celui obtenu au référendum peut-il [166] se muer aussi rapidement en déconfiture et en défaite du colonialisme?

Si on ne se réfère pas constamment à la lutte du peuple algérien on risque de ne pas comprendre précisément l'évolution des rapports entres les colonies et la domination française.

Parce qu'elles se sont révélées incapables de vaincre l'armée nationale algérienne, les forces colonialistes se sont trouvées dépouillées de tout prestige et la crainte qu'elles inspiraient aux peuples colonisés à définitivement disparu. La guerre que mène le peuple algérien depuis quatre ans a fait le lit de l'écroulement français en Afrique. Doréna- 
vant la route est libre pour tous les pays occupés par le colonialisme français.

Le peuple algérien, fidèle, à son serment d'extirper toute trace de domination française en Afrique poursuit le combat.

Le général De Gaulle avant le 15 novembre se trouvera dans l'obligation de reconnaître des Etats autonomes en Afrique Noire.

Dans le même temps, il promet des postes de fonctionnaires aux Algériens.

La misère politique, le paradoxe logique, continuent. Mais c'est la marque du désarroi, de la cécité historique, donc de la défaite. 


\author{
Pour la révolution africaine \\ Écrits politiques
}

Quatrième partie. Vers la libération de l'Afrique.

\title{
Chapitre 15 \\ La guerre d'Algérie et la libération des hommes 30
}

\section{Retour à la table des matières}

Fréquemment l'analyse et l'appréciation d'un événement donné se révèlent inadéquates et les conclusions paradoxales parce que, précisément, il n'a pas été suffisamment tenu compte des liens organiques existant entre cet événement particulier et le développement historique de l'ensemble environnant.

C'est ainsi, pour prendre un exemple, que le renforcement dialectique existant entre le mouvement de libération des peuples colonisés et la lutte émancipatrice des classes ouvrières exploitées des pays impérialistes, connaît quelquefois une sorte de négligence, voire d'oubli.

30 El Moudjahid, No 31 du $1^{\text {er }}$, novembre 1958. 


\section{L'ouvrier et le colonisé...}

Le processus de libération de l'homme, indépendamment des situations concrètes dans lesquelles il se trouve, englobe et concerne l'ensemble de l'humanité. Le combat pour la dignité nationale donne à la lutte pour le pain et la dignité sociale sa véritable signification. Ce rapport interne est l'une des racines de la solidarité immense qui unit les peuples opprimés aux masses exploitées des pays colonialistes.

Au cours des différentes guerres de libération nationale qui se sont succédées pendant ces vingt dernières années, il ne fut pas rare de constater une certaine nuance hostile, voire [168] haineuse de l'ouvrier colonialiste à l'égard du colonisé. C'est que le recul de l'impérialisme et la reconversion des structures sous-développées spécifiques de l'état colonial s'accompagnent dans l'immédiat de crises économiques que les ouvriers du pays colonialiste sont les premiers à ressentir. Les capitalistes «métropolitains se laissent arracher des avantages sociaux et des augmentations de salaires par leurs ouvriers dans l'exacte mesure où l'Etat colonialiste leur permet d'exploiter et de razzier les territoires occupés. Au moment critique où les peuples colonisés se jettent dans la lutte et exigent leur indépendance, il s'écoule une période difficile au cours de laquelle, paradoxalement, l'intérêt des ouvriers et des paysans «métropolitains» semble s'opposer à celui des peuples colonisés. Les méfaits de cette aliénation « inattendue » doivent être connus et énergiquement combattus.

La lutte contre le colonialisme, type particulier d'exploitation de l'homme par l'homme, se situe donc dans le processus général de libération des hommes. Si la solidarité entre ouvriers «métropolitains» et peuples colonisés peut connaître des crises et des tensions, il est rare d'en constater entre peuples colonisés. Les hommes colonisés ont cela en commun qu'on leur conteste le droit de constituer un peuple. Diversifiant et légitimant cette attitude générale du colonialiste, on trouve le racisme, la haine, le mépris chez l'oppresseur et corrélativement l'abétissement, l'analphabétisme, l'asphyxie morale et la sousalimentation endémique chez l'opprimé. 


\section{Solidarité des colonisés}

Entre peuples colonisés il semble exister une sorte de communication illuminante et sacrée qui fait que chaque territoire libéré est pendant un certain temps promu au rang de «territoire-guide». L'indépendance d'un nouveau territoire, la libération des peuples nouveaux sont ressenties par les autres pays opprimés comme une invitation, un encouragement et une promesse. Chaque recul de la domination coloniale en Amérique ou en Asie renforce la volonté nationale des peuples africains. C'est dans la lutte nationale contre l'oppresseur que les peuples colonisés ont découvert, concrètement, la solidarité du bloc colonialiste et la nécessaire interdépendance des mouvements de libération.

L'ébranlement de l'impérialisme anglais, par exemple, ne [169] peut pas réellement s'accompagner d'une consolidation de l'impérialisme français. Dans l'immédiat, pareil résultat peut paraître évident. En réalité, le flux national, l'émergence de nouveaux Etats, préparent et précipitent le reflux inévitable de la cohorte colonialiste internationale. L'apparition de peuples hier inconnus sur la scène de l'histoire, leur volonté de participer à l'édification d'une civilisation à la mesure du monde, donne à la période contemporaine une importance décisive dans le processus d'humanisation du monde.

Le pacte de Bandoeng concrétise à la fois cette union charnelle et spirituelle des peuples colonisés. Bandoeng est l'engagement historique des hommes opprimés à s'entraider et à imposer un recul définitif aux forces d'exploitation.

\section{L'Algérie « territoire-guide »}

La guerre d'Algérie occupe une place de choix dans le processus de démolition de l'impérialisme. Depuis quatre ans, le colonialisme français, l'un des plus obstinés de cet après-guerre, s'accroche par tous les moyens à sa tête de pont en Afrique. Tous les arguments militaires et 
politiques ont été utilisés pour justifier la répression et la présence française en Algérie. Les dimensions de cette guerre atroce ont étonné et bouleversé l'opinion internationale. Le colonialisme français en Algérie a mobilisé toutes ses forces.

L'effort militaire, économique et politique déployé par la France dans la guerre d'Algérie ne peut s'apprécier objectivement qu'en fonction de l'ensemble africain « français ». Vaincre la Révolution algérienne, c'était bien sûr expurger pour une dizaine d'années encore le «ferment nationaliste ». Mais c'était à la fois imposer le silence aux éventuels mouvements africains de libération et surtout marquer du sceau de la débilité et de l'insécurité les jeunes indépendances tunisienne et marocaine.

Le colonialisme français en Algérie a considérablement enrichi I'histoire des méthodes barbares utilisées par le colonialisme international. Pour la première fois, l'on assiste à la mobilisation de plusieurs classes, à l'envoi des contingents, à la diminution des forces de défense nationale, au bénéfice d'une guerre de reconquête coloniale. À plusieurs reprises, les gouvernants français ont annoncé une imminente victoire sur les forces nationales algériennes. Toutes les conditions objectives [170] semblaient être réunies pour réaliser cette défaite de la révolution algérienne. Chaque fois on a assisté à une sorte de miracle, de renouveau, de recommencement.

C'est que le peuple algérien se sait soutenu par d'immenses forces démocratiques internationales. De plus, les masses algériennes sont conscientes de l'importance de leur combat à l'échelle du continent africain.

La guerre d'Algérie est loin d'être terminée et à l'aube de cette cinquième année de guerre, les hommes et les femmes d'Algérie habités par une faim incoercible de paix, mesurent lucidement le chemin très difficile qui leur reste à parcourir. Mais les résultats positifs, décisifs, irréversibles que leur lutte vient de rendre possibles en Afrique alimentent leur foi et renforcent leur combativité.

Alors que la Tunisie et le Maroc, protectorats, ont pu accéder à l'indépendance sans mettre fondamentalement en question l'empire français, l'Algérie, par son statut, l'ancienneté de l'occupation et l'importance de l'implantation colonialiste, pose au grand jour et de façon critique la question de l'écroulement de l'empire. 
Pour le colonialisme français, l'Algérie n'est pas uniquement un nouveau conflit colonial mais aussi l'occasion d'une décisive confrontation, le test-limite. Aussi les forces françaises réagirent-elles au cours de ce conflit, avec une brutalité et une violence qui ont souvent déconcerté. Le conflit franco-algérien a posé le problème colonial à l'échelle de l'Afrique. Les autres puissances coloniales en Afrique suivent avec anxiété et terreur l'évolution de la guerre de l'Algérie. Et de l'autre côté du Sahara voici que la Guinée indépendante allonge maintenant son ombre « subversive » vers les territoires les mieux tenus.

L'Algérie, tête de pont du colonialisme occidental en Afrique est rapidement devenue le guêpier où s'est enlisé l'impérialisme français et où se sont écroulés les espoirs insensés des oppresseurs occidentaux.

La guerre d'Algérie depuis quatre ans domine de façon tragique et décisive la vie politique intérieure et extérieure française. Les rapports de la France avec les autres pays occidentaux, ses difficultés diplomatiques ou quelquefois militaires avec les Etats arabes, l'évolution des structures colonialistes [171] de la vieille union française reflètent de façon très nette les différentes phases de la guerre d'Algérie.

Obsédés par la terreur de nouvelles guerres coloniales, les hommes politiques français ont multiplié les mises en garde et les invitations: repensons nos problèmes avec nos possessions coloniales, telle est la phrase qui à partir de 1955 résonne dans les assemblées françaises et dans les milieux politiques. La Loi-Cadre de M. Defferre fut créée dans le dessin d'éviter des revendications nationales intempestives.

Mais l'existence de la guerre d'Algérie, les détails sur la répression colonialiste, l'héroïsme du peuple algérien ont éveillé et enhardi la conscience des hommes et des femmes d'Afrique.

Au début de 1958, dans tous les territoires africains occupés par la France, la volonté nationale se fait jour et des partis de plus en plus nombreux et de plus en plus décidés posent le problème nécessaire de la lutte armée.

Au Togo et au Cameroun, les événements ont effectivement pris l'allure de guerre larvée. Ailleurs, les garde-chiournes du colonialisme multiplient les déclarations lénifiantes. Or, l'observateur décèle parfaitement sous ces affirmations tranquilles une intense anxiété et une terreur de la colère populaire. 
La guerre d'Algérie a secoué en profondeur l'équilibre colonial en Afrique. Il n'y a pas un territoire occupé en Afrique qui n'ait été remanié dans ses perspectives d'avenir par la guerre d'Algérie. Le peuple algérien est conscient de l'importance du combat dans lequel il est engagé. Depuis 1954 il a posé comme mot d'ordre la libération nationale de l'Algérie et la libération du continent africain. Les faciles critiques contre le refus rigide d'accepter des étapes dans la décolonisation adressées périodiquement au F.L.N. ne tiennent pas suffisamment compte des dimensions originellement africaines de la lutte nationale algérienne.

\section{Le colonialisme français doit mourir}

L'appel au général De Gaulle fut, dans ces conditions, la dernière tentative du colonialisme français. On a vu que le général De Gaulle n'a pu que tirer les conséquences d'un mouvement qui le dépasse. La nouvelle constitution, dans ses considérations [172] sur la communauté laisse encore une place de choix à la métropole, mais admet l'indispensable reconnaissance d'Etats autonomes. La création de la République malgache est la première manifestation de cette réforme gaulliste.

Les milieux colonialistes qui avaient mis leur confiance dans l'intercession du général providentiel en arrivent à se demander aujourd'hui s'ils n'ont pas fait un marché de dupes. Faute d'avoir tiré les conclusions d'une exigence irréversible qui, si elle n'était satisfaite, risquerait d'engloutir la France elle-même, les colonialistes français ont tendance à considérer De Gaulle comme un traître ou comme un bradeur. En vérité le général sauve encore une fois les intérêts colonialistes en disposant une communauté qui, inégale, organisée au seul profit d'une métropole, maintient intactes d'importantes structures coloniales. 
Avec la constitution d'Etats autonomes le colonialisme français sort affaibli. Mais, sans l'intervention du général De Gaulle c'était l'éclatement de l'empire à très brève échéance. Apparemment traître à ses mandats, le général De Gaulle est en réalité le sauveur momentané d'une certaine réalité coloniale. 


\section{Pour la révolution africaine Écrits politiques}

Quatrième partie. Vers la libération de l'Afrique.

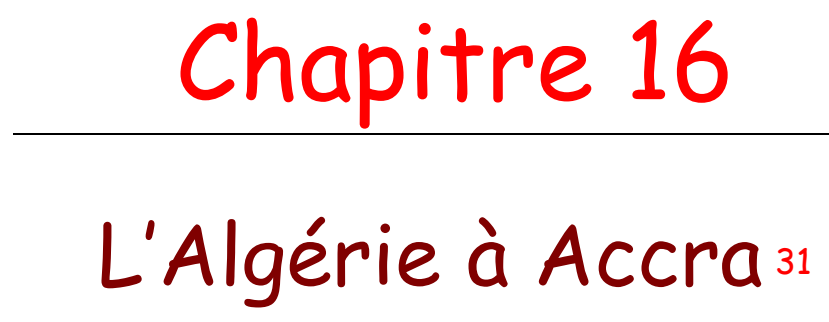

\section{Retour à la table des matières}

La délégation algérienne composée de cinq membres a reçu à Accra un accueil enthousiaste. Cette réception chaleureuse témoignait de l'importance accordée par les peuples africains depuis plusieurs années.

Nous nous sommes aperçus à Accra que les grandes figures de la Révolution algérienne Ben Bella, Ben M'Hidi, Djamila Bouhired sont entrées dans l'épopée africaine.

Une place de choix a été faite à plusieurs membres de notre délégation. C'est ainsi que l'un de nous a siégé au Comité-Directeur du Congrès et que tous les autres ont été élus par acclamation à la présidence ou à la vice-présidence des différentes commissions.

Une telle unanimité à l'égard de l'Algérie combattante a manifestement déplu aux colonialistes qui s'imaginent que la lutte du peuple algérien n'éveillait pas d'écho chez les hommes et les femmes 
d'Afrique Noire. En réalité, la Révolution algérienne n'a jamais été présente avec tant d'acuité et de poids que dans cette région d'Afrique: qu'il se soit agi des Sénégalais, des Camerounais ou des Sud-Africains, il était facile de constater l'existence d'une solidarité fondamentale de ces peuples avec la lutte du peuple algérien, ses méthodes ses objectifs.

La délégation algérienne a posé de façon très claire devant les congressistes le problème de la lutte armée. Certains observateurs, certains journalistes aux premières heures du [174] Congrès s'étaient crus autorisés à câbler à leurs journaux que l'Algérie avait décidé de mener le combat anti-colonialiste par des méthodes pacifiques.

Quelques-uns n'hésitaient d'ailleurs pas à laisser entendre qu'on assistait officiellement à une condamnation du mouvement révolutionnaire algérien.

Or, dès le premier jour, le Congrès était mis dans son orbite authentique et la lutte algérienne devenait à la fois le point faible du système colonial et le rempart des peuples africains.

C'est que les congressistes se sont rapidement convaincus que l'intérêt des colonialistes pour l'Afrique et les amorces de décolonisation apparues çà et là, ne sont pas le fait de la générosité ou de la subite intelligence des oppresseurs.

La guerre d'Algérie a pesé de façon décisive dans ce congrès car, pour la première fois, un colonialisme qui fait la guerre en Afrique se révèle impuissant à vaincre. C'est faute d'avoir analysé ce phénomène que les colonialistes se sont encore une fois étonnés du succès des représentants algériens.

Chaque délégué algérien fut reçu comme celui qui est en train d'expulser de la chair du colonisé la peur, le tremblement, le complexe d'infériorité.

La lutte du peuple algérien n'est pas saluée comme un acte d'héroïsme mais comme une action continue, soutenue, en perpétuel renforcement et qui contient dans son développement l'effondrement et la mort du colonialisme français en Afrique.

La Guinée fut également applaudie mais c'était comme la première conséquence importante du conflit franco-algérien. 
D'ailleurs, les camarades ministres de la Guinée présents à la Conférence nous ont demandé de faire connaître à notre gouvernement la reconnaissance profonde du peuple guinéen envers l'Algérie combattante.

D'autre part, le premier ministre du Ghana, le Dr N'Krumah a tenu à recevoir nos délégués parmi les premiers. Pendant plus d'une heure, le problème algérien dans ses rapports avec la libération du continent africain a été étudié. Encore une fois le Chef de l'Etat du Ghana a renouvelé au peuple algérien [175] en lutte l'appui et la solidarité agissante du peuple du Ghana et de son gouvernement.

Le Dr N'Krumah nous a fait part de l'intention de son gouvernement de reconnaître prochainement le Gouvernement Provisoire de la République Algérienne. 


\section{Pour la révolution africaine \\ Écrits politiques}

Quatrième partie. Vers la libération de l'Afrique.

\section{Chapitre 17}

\section{Accra: I'Afrique affirme son unité et définit sa stratégie 32}

\section{Retour à la table des matières}

En 1884, les nations occidentales réunies à Berlin décidaient de se partager le continent africain et fondaient légalement le régime colonial.

Depuis l'évolution des rapports de force dans le monde, la naissance de nouvelles puissances, ont obligé les nations occidentales à reculer et à se retirer d'une grande partie de leurs possessions.

\section{Après l'Asie, l'Afrique}

L'Asie est maintenant libérée du colonialisme et des territoires comme la Chine, frappés semble-t-il jusqu'à ce jour d'une sorte de misère absolue, créent comme une nouvelle civilisation, authentique cellelà, qui concerne l'homme et qui débouche immanquablement sur lui.

32 El Moudjahid, № 34 du 24 décembre 1958. 
Il reste que le continent africain est encore largement occupé par les puissances coloniales et après Bandoeng, après la conférence afroasiatique du Caire voici que les peuples africains se réunissent à Accra, capitale du Ghana indépendant pour jeter les bases, dans la perspective lointaine des Etats-Unis d'Afrique, d'une tactique et d'une stratégie de combat.

[178]

\section{Une solidarité « biologique »}

À Accra se sont retrouvées les organisations politiques et syndicales du continent africain: leur idéologie commune: volonté nationale contre la domination étrangère : leur tactique : affaiblir les uns après les autres colonisateurs ; leur stratégie: déjouer les manœuvres et les tentatives de camouflage de l'oppresseur.

Ce qui a frappé l'observateur à Accra, c'est l'existence au niveau le plus spontané d'une solidarité organique, biologique même. Mais audessus de cette sorte de communion affective il y avait bel et bien le souci d'affirmer une identité d'objectifs et aussi la volonté d'utiliser tous les moyens existants pour expulser le colonialisme du continent africain.

Ces hommes et ces femmes s'étaient réunis à la fois pour exposer la nature du colonialisme auquel ils étaient soumis, pour étudier le type de lutte possible, pour articuler leurs offensives, pour faire pression enfin, territoire après territoire, sur des colonialismes identiques.

C'est pourquoi très rapidement par delà les commissions, se sont créés des contacts entre pays sous tutelle, colonies de peuplement du type Afrique du Sud, Kenya, Algérie, Etats dans la communauté représentée essentiellement par les Afriques dites françaises.

Les Etats indépendants d'Afrique ont reçu à Accra un accueil enthousiaste. Ce sont ces Etats qui en avril 1958, avaient jugé indispensable cette réunion d'Accra pour accélérer la libération du continent africain. 
La R.A.U., la Tunisie, le Ghana, I'Ethiopie, etc... avaient tenu à déléguer au Congrès des hommes et des femmes qui témoignaient de l'appui inconditionnel de ces Etats aux différents peuples en lutte.

La jeune République guinéenne représentée par trois de ses ministres, fut acclamée avec enthousiasme par le Congrès.

\section{Violence et non-violence: la fin et les moyens}

Plusieurs problèmes ont été débattus au cours de cette Conférence.

Les deux plus importants semblent avoir été celui de la [179] nonviolence et celui de la collaboration avec l'ex-nation dominatrice.

Il est évident que les problèmes sont liés. La fin du régime colonial réalisée selon des formes pacifiques et rendue possible par la compréhension du colonialiste pourrait dans certaines circonstances déboucher sur une collaboration renouvelée des deux nations. Or l'histoire montre que nulle nation colonialiste n'accepte de se retirer sans que toutes ses possibilités de maintien n'aient été épuisées.

Poser le problème d'une décolonisation non-violente, c'est moins postuler une soudaine humanité du colonialiste que croire à la pression suffisante du nouveau rapport de forces à l'échelle internationale.

Il est clair par exemple que la France a amorcé un processus de décolonisation en Afrique Noire.

Cette innovation sans violence a été rendue possible par les échecs successifs du colonialisme français dans les autres territoires. Toutefois, les représentants des nations africaines sous domination française présents à Accra, ont dénoncé avec lucidité les manœuvres de l'impérialisme français. 


\section{Les pièges du néo-colonialisme}

Les congressistes ont condamné sans réserve les Africains qui, pour se maintenir, n'ont pas craint de mobiliser la police pour truquer les élections au dernier référendum et pour engager leurs territoires dans une association avec la France qui exclut pour de longues années la voie de l'indépendance. Les quelques délégués venus représenter ces gouvernements fantoches de l'Afrique française se sont vus plus ou moins expulser des commissions.

Par contre les représentants du Cameroun à la tête desquelles se trouvait le Dr Félix Moumié ont été chaleureusement applaudis au cours de la dernière Session de l'O.N.U. D'autres territoires ont arraché leur indépendance à terme : le Cameroun, le Tanganyka, la Somalie.

En 1960, près de 60 millions d'Africains seront de nouveau indépendants.

De plus, le gouvernement belge alarmé par les soubresauts qui agitent l'Afrique et par le durcissement des mouvements nationalistes du Congo Belge vient officiellement de reconnaître [180] la vocation nationale du Congo Belge et se propose au mois de janvier de présenter un programme par étapes pour l'indépendance de 20 millions de Congolais.

Il n'est pas exclu que les colonialistes belges essaient encore une fois de retarder cette échéance : il faut faire confiance aux masses congolaises pour imposer dans un délai rapproché la République Démocratique du Congo.

Si la Belgique, l'Angleterre avec le Nigéria et le Tanganyka, la France avec la Guinée ont reculé, le Portugal développe au contraire le régime policier dans ses possessions. Les délégués de l'Angola ont été accueillis avec émotion et une immense colère s'est exprimée à l'audition des mesures discriminatoires et inhumaines employées par les autorités portugaises. De toute évidence, l'Angola, l'A frique du Sud et l'Algérie sont les citadelles du colonialisme et probablement les territoires dans lesquels le peuplement européen se défend avec le plus d'acharnement et de férocité. 
À ce propos, il faut signaler que I'Union Sud-Africaine tente d'annexer le Basutoland, le Swiziland et de faire la jonction avec les Rhodésies, autres colonies de peuplement.

Cette colonisation après la colonisation est certainement l'un des phénomènes les plus remarquables de cette période de libération du continent.

\section{La légion africaine}

Dans les colonies de peuplement du type Kenya, Algérie Afrique du Sud, l'unanimité s'est faite: seule la lutte armée provoquera la défaite de la nation occupante. Et la légion africaine dont le principe a été adopté à Accra est la réponse concrète des peuples africains à la volonté de domination coloniale des Européens.

Les peuples africains en décidant la création dans tous les territoires d'un corps de volontaires, entendent manifester clairement leur solidarité aux autres peuples, exprimant ainsi que la libération nationale est liée à la libération du continent.

Les peuples en lutte, convaincus aujourd'hui que leurs frères africains partagent leur combat et sont prêts à intervenir directement au premier appel de leurs organismes dirigeants voient l'avenir de façon plus sereine et plus optimiste.

Dans les meetings populaires organisés au Ghana, en Éthiopie, [181] au Nigéria, des centaines d'hommes ont fait le serment de se porter au secours de leurs frères algériens ou Sud-Africains dès que ceux-ci en manifesteront le désir.

L'Afrique doit être libre, a dit le Dr N'Krumah dans son discours inaugural, nous n'avons rien à perdre que nos chaînes et nous avons à conquérir un continent immense. A Accra, les Africains se sont jurés fidélité et assistance. Aucune alliance ne sera rejetée, l'avenir du colonialisme n'a jamais été aussi sombre qu'au lendemain de la Conférence d'Accra. 


\section{Pour la révolution africaine \\ Écrits politiques}

Quatrième partie. Vers la libération de l'Afrique.

\section{Chapitre 18}

\section{Les tentatives désespérées de M. Debré ${ }_{33}$}

\section{Retour à la table des matières}

M. Michel Debré, chef du gouvernement français s'est rendu dernièrement en Algérie. Il a pris contact avec les autorités colonialistes et défini à leur intention, le programme de son gouvernement.

"L'autorité de la France en Algérie, dira-t-il, est une exigence de I'histoire, de la nature, de la morale ». Cette déclaration, éclairée par des prises de positions plus fermes telles que cette phrase prononcée devant les Anciens combattants: «Il faut que tous les Algériens sachent et comprennent définitivement que chaque habitant de ce pays est citoyen français au même titre que n'importe quel citoyen de la métropole et qu'en aucun cas le gouvernement n'accepterait que cette citoyenneté soit remise en question $»$, ou cette autre contenue dans l'allocution personnelle du Président du conseil français: «La vérité, 
c'est que l'Algérie est terre de souveraineté française » indique à la fois la non modification de la population française en Algérie et l'importance du hiatus qui existe actuellement entre la volonté nationale du peuple algérien et l'obstination colonialiste française.

\section{Un temps historique falsifié}

Le programme de M. Debré se situe dans un dogmatisme colonialiste éminemment orthodoxe.

La conquête crée des liens historiques, affirme-t-on. Le [184] temps nouveau inauguré par la conquête, ce temps colonialiste parce que habité par des valeurs colonialistes, parce que tirant sa raison d'être de la négation du temps national va se trouver affecté d'un coefficient absolu. L'histoire de la conquête, le développement historique de la colonisation et du dépouillement national vont se substituer au temps réel des hommes exploités. Et ce qui sera affirmé par les colonisés au moment de la lutte de libération nationale comme volonté de rompre avec l'exploitation et le mépris, sera rejeté, par la puissance colonialiste comme symbole de barbarie et de régression.

C'est que le colonialiste, par un mécanisme de pensée somme toute assez banal, en arrive à ne plus pouvoir imaginer un temps se faisant sans lui. Son irruption dans l'histoire du peuple colonisé est déifiée, transformée en nécessité absolue. Or un «regard historique sur l'histoire » exige au contraire que le colonialiste français se retire car il est devenu historiquement nécessaire que le temps national en Algérie existe.

L'exigence historique de M. Debré est l'équivalent intellectualisé de la vieille formule colonialiste: "C'est nous qui avons fait l'Algérie ».

En réalité, ce qui se donne comme fidélité à l'histoire n'est en dernière analyse qu'infidélité à l'histoire, que refus d'être à la mesure de la période de décolonisation, que désobéissance à l'histoire.

En 1959, le sens de l'histoire exige que les 10 millions d'Algériens prennent leur destinée en mains. Depuis plus de quatre ans, les gouvernements français successifs se sont révélés incapables d'interpréter objectivement ce problème. Les déclarations de M. Debré ne diffèrent 
en rien de celles prononcées par $M$. Léonard ou $M$. Soustelle: «La France est en Algérie et y restera », disait-ils en 1954.

En 1959, « la France demeurera».

Un autre thème est généralement évoqué par les doctrinaires du colonialisme, c'est celui de l'union indissoluble de l'Algérie et de la France.

\section{Une géographie d'intentions}

Ne pouvant être ni sentimentale, ni intellectuelle cette union sera géographique. Et tantôt la France se trouve être le prolongement européen de l'Algérie, tantôt l'Algérie le prolongement africain de la France.

[185]

Les difficultés internationales de l'Europe vont donner à cette union géographique une primauté de plus en plus marquée. Dans le cadre du système défensif de l'Occident le territoire algérien occupe une place privilégiée. C'est ce que feront ressortir les différents défenseurs de l'Algérie française. C'est également dans ce sens que le problème sera évoqué par $M$. Debré : « Nous devons d'autre part, comprendre que la liberté et le progrès, la sécurité et la paix sont liés dans cette partie du monde à l'unité, au-dessus de la Méditerranée de la France métropolitaine porte de l'Europe et de l'Algérie tête de l'Afrique. Toute atteinte à cette unité est un risque d'insécurité! Tout renforcement de cette unité est un gage de paix. La France se doit donc de faire que nul ne puisse douter de sa volonté de rendre cette unité plus ferme que jamais ».

Ainsi l'Algérie doit rester terre française car les nécessités stratégiques de l'Europe et de la France l'exigent. La géographie de M. Debré est donc une géographie animée d'intentions : l'autorité de la France en Algérie est une exigence de la nature. C'est l'ordre naturel qui impose à la France le maintien du régime colonial en Algérie. Dès que les régimes ou les hommes commencent à lire leurs actions politiques dans les sinuosités du terrain, ce sont le fascisme et le nazisme qui se trouvent en question. 
C'est en prolongeant par l'imagination, certaines lignes du terrain que les gouvernements remettent en cause la paix du monde. Qu'ils veuillent réellement donner libre cours à leurs fantaisies et étendre ce que depuis plusieurs siècles on appelle les frontières naturelles, et voici des peuples entiers, jetés dans le sang et la misère.

La géographie de l'Algérie, ce ne devrait être un secret pour personne, réclame d'abord que ce pays soit indépendant.

Et après, certes, elle a sa place à prendre dans le Maghreb en Afrique et dans le Monde. Mais nier le destin national de l'Algérie au nom d'une "plus grande union franco-algérienne » est de l'imposture. En cela M. Debré n'innove pas. Depuis quatre ans, les traditions sont établies dans les gouvernements français.

Echappant à l'histoire et à la nature, M. Debré débouche sur la morale. Là encore il retrouve, sans effort semble-t-il, les grands principes de l'ultra colonialisme: "Que feront les Algériens sans nous?» Les colons de la Mitidja disaient et disent [186] encore: « Ces champs de vignes, en 4 ans, seront transformés en marécages ».

M. Debré ne dit pas autre chose: «On veut installer l'Algérie dans la misère, dans la barbarie et dans le sang ».

\section{La morale au secours de l'exploitation}

Or, autre thème, seule la France est capable de prendre en charge bénéfiquement l'Algérie.

Seule la France peut prendre économiquement la charge de l'Algérie.

Tout cela, M. Debré le redira à Alger.

«La France a une obligation d'ordre économique... La France a une obligation d'ordre social... La France a une obligation humaine car elle seule est capable de maintenir et de renforcer... cette union et même fraternité des esprits qui donnent à l'Algérie un caractère et une force unique au monde. » 
Signe des temps! Au début de la conquête, c'est ce prétexte qui était évoqué. Lutte contre la barbarie, la misère l'arriération. Aujourd'hui, après 130 années d'exploitation, qui constituent paradoxalement un droit, et la lutte des blocs, c'est l'histoire et la stratégie qui prennent la première place.

La morale française, les valeurs françaises sont seules capables de maintenir l'Algérie dans le camp des « régions humaines». Le départ de la France, nous prévient $M$. Debré, serait le signe d'une replongée de l'Algérie dans l'archaïsme, l'arriération et l'abêtissement.

Huit mois après la prise du pouvoir par le général De Gaulle, nous voici revenus aux premiers mois de la lutte de libération de notre peuple: «La France est chez elle en Algérie car l'Algérie est l'œuvre de la France. La France a besoin de l'Algérie car sans l'Algérie que ferait la France. L'Algérie a besoin de la France car sans la France que ferait l'Algérie. »

Et pour conclure, cette précision contenue dans l'allocution personnelle de M. Debré : «Qui peut douter, sinon de mauvais esprits, avec des arrières-pensées, des résolutions du général De Gaulle? Qui ose, sinon de mauvais esprits, avec des arrières-pensées, douter lorsque le général De Gaulle a déclaré qu'il n'y aurait pas de négociations politiques?»

La Ve République française ne semble pas faire preuve de plus d'imagination que la précédente. Les mêmes affirmations [187] sont reprises avec la même obstination aveugle, le même mépris des événements, la même intelligence de l'histoire.

Prétendre en 1959 que tous les Algériens sont français, que la France demeurera en Algérie et qu'il ne saurait y avoir de négociations politiques, c'est s'engager en dépit du bon sens dans la voie de l'intensification de la guerre.

C'est incontestablement tourner le dos à la négociation et à la raison.

Il est vrai que cet aveuglement n'est pas le résultat d'une erreur d'appréciation. La France et son gouvernement sont encore dominés par des intérêts colonialistes. Or, depuis le 13 mai, nous assistons à l'alliance de ces intérêts traditionnels avec la poussée fasciste et mili- 
tariste toujours très forte en France (Boulanger, De La Rocque, Pétain...) et d'une certaine fraction du grand capital.

Tant que cette alliance n'aura pas été mise en cause, les présidents du conseil français sont condamnés à s'inspirer dans leurs déclarations de la tradition ultra-colonialiste.

Il faut savoir que le temps est loin qui verra l'éclatement, par le développement des contradictions, du bloc colonialiste.

Aujourd'hui comme depuis 4 ans, le mot d'ordre est toujours d'intensifier la lutte armée. Toutes les tentatives de diversion de l'adversaire doivent être anéanties.

Les conditions d'une négociation sont bien loin d'être réunies du côté français. Le programme de M. Debré est de faire la guerre, de nier la nation algérienne, d'approfondir l'annexion de notre pays.

Oui, comme le disait M. Yazid, ministre de l'Information parler de souveraineté française en Algérie en 1959, c'est de la folie. Il n'y a pas d'autre mot. 


\author{
Pour la révolution africaine \\ Écrits politiques
}

Quatrième partie. Vers la libération de l'Afrique.

\title{
Chapitre 19
}

\section{Fureur raciste en France ${ }^{34}$}

\section{Retour à la table des matières}

Il y a deux ans, à la suite de l'action des commandos de l'A.L.N. sur le territoire français, soit pour neutraliser la contre-révolution alors très active, soit pour réagir contre certains tortionnaires de la police française, on vit se développer des attitudes spontanées de racisme et de discrimination passionnelle à l'égard des Nord-Africains - De façon immédiate et globale la méfiance envers les Arabes devient une seconde nature. Un pas de plus et la chasse fut ouverte. - C'est la période, on s'en souvient où même un Sud-Américain était criblé de balles car il ressemblait à un Nord-Africain.

Les citoyens tunisiens et les citoyens marocains devaient souffrir également de cette conduite raciste. Ouvriers tunisiens arrêtés, internés, passés à tabac... étudiants marocains appréhendés, interrogés dans les locaux de la police judiciaire... Dans les rues, remarques désobligeantes, humiliantes et pour la personne et pour les jeunes Etats. 
C'est la période, on s'en souvient, où tout Nord-Africain était plusieurs fois interpellé et où de nombreux ouvriers tunisiens ou marocains travaillant en France décidaient de rejoindre le territoire national.

À cette occasion l'habitude fut prise de décréter que seuls les Algériens étaient responsables de cet état de choses et qu'il dépendait des Algériens de faire cesser cette suspicion généralisée à l'égard de la population nord-africaine.

[190]

Ainsi donc ce comportement fortement agressif et haineux n'était pas une composante des structures sociales et mentales du peuple français, mais simplement la réaction d'auto-défense d'organisme qui faisait difficilement la différence entre les habitants du Maghreb.

Les récents événements qui se sont déroulés sur le territoire français méritent d'être évoqués ici. Ils nous montreront sans nul doute que la confusion dans la perception du « métèque » n'était pas à mettre sur le compte d'une ignorance regrettable, mais trouvait sa justification dans un principe, banal celui-là, qui veut que les formes les plus brutales du racisme s'installent en France à une cadence véritablement explosive.

\section{Un écrivain poignardé}

Le premier fait est l'attentat dirigé contre le jeune écrivain Oyono, il y a trois semaines. L'auteur d' "Une vie de Boy » sortait d'un restaurant en compagnie d'une femme. Le couple fut pris d'assaut, la femme insultée de façon obscène, giflée, piétinée. Quant à Oyono, après avoir résisté à ses assaillants, il devait s'écrouler, un poignard planté entre les omoplates et admis d'urgence dans un hôpital parisien. Après une transfusion sanguine, il devait se remettre. Aujourd'hui rétabli, il dit à qui veut l'entendre qu'il ne se sent pas en sécurité sur le territoire français et prépare son départ.

Comment interpréter cet événement? Il ne s'agit pas d'un attentat dirigé contre cet homme de couleur, ni d'une tentative de vol. Il s'agit, les auteurs l'ont suffisamment proclamé, d'une correction donnée à la 
femme (qui était blanche), et d'un avertissement donné au nègre. La meute s'est jetée sur Oyono aux cris de «A mort les nègres! 》.

Oyono avant de s'évanouir avait pu remettre à la police l'un de ses agresseurs. On ignore jusqu'à ce jour les noms des assaillants et malgré des prises de positions fermes de plusieurs associations, il est douteux qu'une suite judiciaire soit donnée à cette affaire. A signaler que cela se passait en plein quartier latin, c'est-à-dire en plein quartier intellectuel habitué à la présence d'étudiants de toutes origines. Personne ne s'est porté [191] au secours de l'agressé et, l'opération terminée, les assaillants ont pu tranquillement disparaître.

\section{Un film anti-raciste pris à partie...}

Dans le souci de lutter contre le racisme ambiant aux Etats-Unis et ailleurs, plusieurs films ont déjà été tournés. Le dernier en date « Tripes au soleil » sur lequel on pourrait dire beaucoup de choses vient d'être projeté à Paris. Dès la première séance, des jeunes parisiens déchaînèrent un vacarme extraordinaire, brisèrent des fauteuils, souillèrent l'écran et manifestèrent leur hostilité à la fin de la séance. Les cris $\dot{A}$ bas les nègres, à mort les nègres, vive Hitler, furent lancés et la police « dispersa les manifestants».

Depuis plusieurs semaines, de façon systématique, les organisations anti-fascistes sont prises à partie. L'un des mouvements les plus actifs, le M.R.A.P., contre le racisme, l'antisémitisme et pour la paix, qui l'un des premiers a pris position contre le principe de la guerre d'Algérie et pour la reconnaissance de la nation algérienne, est l'objet d'attaques ininterrompues. Ses locaux sont assaillis presque quotidiennement et ses membres dirigeants l'objet de menaces et de voies de fait. Sur les murs de Paris, depuis quelques semaines également, des croix gammées ont fait leur apparition. Ces croix à Paris ne sont que les répliques de celles qu'on peut voir depuis plus longtemps à Alger et à Constantine.

Lorsque en France, un film anti-raciste est pris à partie, en plein jour par une organisation qui ne craint pas de proclamer le slogan : A mort les nègres! on peut dire que la démocratie va très mal en France et que les nègres feraient bien de quitter le navire. 
Mais alors, dit-on, ne faut-il pas se garder de généraliser à partir de ces faits?

Ne s'agit-il pas simplement de manifestations épisodiques réprimées par la loi et unanimement condamnées par la conscience nationale française? Peut-être convient-il de s'attarder davantage. Et peutêtre que de telles manifestations ne surgissent pas spontanément. Pour qu'elles existent, pour qu'elles prennent corps il faut que dans une certaine mesure, dans la conscience nationale précisément, il se soit produit une sédimentation suffisante de racisme, de complexe de supériorité, [192] de discrimination. Ces manifestations, issues en droite ligne du cœur, c'est-à-dire du cœur de l'individu, expriment et le vice de l'éducation française à l'égard du reste de l'humanité, et aussi, les conséquences de dizaines d'années de domination coloniale.

Le général De Gaulle lui-même, lors de son dernier discours à Blois, n'a-t-il pas, miraculeusement, retrouvé ce chemin du cœur?

Parlant de la nécessité de trouver un accord en Europe, n'a-t-il pas dit que « nous blancs et civilisés, nous nous devions de trouver un terrain d'entente? $»$.

On pense immanquablement à ce passage du poète noir Césaire :

«Ce qu'il (le bourgeois humaniste du $X X^{e}$ siècle) ne pardonne pas à Hitler, ce n'est pas le crime en soi, le crime contre l'homme blanc, c'est d'avoir appliqué à l'Europe des procédés colonialistes dont ne relevaient jusqu'ici que les Arabes d'Algérie, les coolies de l'Inde et les nègres d'Afrique ».

Oui, lorsque le racisme atteint en France de telles dimensions, il est temps que les nègres quittent le navire. Aux membres de la «Communauté française » de décider si leur place est encore aux côtés de ceux qui ne sont pas encore débarrassés à l'égard de la race noire ni des bassesses ni de la haine. 
[193]

\author{
Pour la révolution africaine \\ Écrits politiques
}

Quatrième partie. Vers la libération de l'Afrique.

\title{
Chapitre 20
}

\section{Le sang coule aux Antilles sous domination française ${ }_{35}$}

\section{Retour à la table des matières}

Ainsi donc les vieilles colonies elles aussi empruntent le chemin de la « rébellion». Ces fleurons de l'empire, ces pays castrés qui donnèrent tant de bons et loyaux serviteurs se mettent à bouger.

Tout Antillais, tout Guyannais où qu'il se trouve aujourd'hui se sentira ébranlé de façon violente. Les Français en effet après avoir péjorativement catégorisé les Arabes et les Africains, les Malgaches et les Indochinois concédaient, reconnaissaient qu'avec les Antillais les choses prenaient une autre tournure. Les Antillais entendait-on de partout, sont des Français, comme les Corses. Et il y avait d'énormes masses d'Antillais et d'Antillaises à le croire. Bien sûr de temps à autre le racisme éclatait, bien sûr le colonat antillais opprimait et condamnait à la famine endémique les ouvriers agricoles mais le titre 
de citoyen français valait bien ces quelques désagréments. Bien sûr chaque année trois cents tonnes d'or quittaient le territoire guyannais pour renflouer les caves de la Banque de France mais M. Monnerville deuxième ou troisième citoyen français n'était-ce pas à la fois un symbole et le paiement d'une dette?

Pourtant malgré cette intoxication immense, en dépit de cette énorme imposture, il s'est trouvé des Martiniquais pour [194] entrer en lutte ouverte contre les forces françaises, investir des commissariats, couper des routes. Submergeant ces trois cents ans de présence française il s'est trouvé des Martiniquais à sortir leurs armes et à occuper Fort-de-France pendant plus de six heures. Des morts! Il y en a eu. Des blessés aussi.

Quinze morts, nous dit-on, plusieurs dizaines de blessés et des centaines d'arrestations.

Des renforts sont acheminés en direction des Antilles et afin de briser un mouvement qui doit paraître suffisamment imminent on inonde la Guadeloupe de fusiliers-marins, de C.R.S. et de soldats.

Les services français d'information prétendent que l'origine de l'émeute serait un banal incident de circulation. Peut-être. Mais alors pourquoi cette subite ampleur? D'où vient qu'une population réagisse avec tant de violence, tant de rage. D'où vient que les C.R.S. réagissent avec tant de précipitation tant de désinvolture pour la vie de « concitoyens ».

En réalité le problème est posé. Et c'est tant mieux. La fiction Antilles françaises, la formule «pour l'Antillais, il n'y a pas de problème » sont remis en question. Et c'est tant mieux.

Les vieux politiciens, assimilés, infestés du dedans, qui depuis longtemps ne représentaient que leurs intérêts médiocres et leur propre médiocrité doivent aujourd'hui être très inquiets. Ils découvrent soudain que des Martiniquais peuvent parfaitement être traités en rebelles par la France. Ils découvrent aussi l'existence d'un esprit rebelle, d'un esprit national.

Lors du référendum organisé par la France on avait demandé à Césaire la raison de son Oui à De Gaulle. C'est que, avait-il répondu, les Martiniquais ont conclu un pari avec la Ve République. Notre Oui, disait 
Césaire est un Oui circonstancié. La France s'engage à améliorer notre condition et à nous reconnaître certaines prérogatives sur le plan local.

Eh bien! il semble que le peuple remette ce pari en cause et pose le problème national. La question antillaise, la question de la fédération caraïbe ne peut plus être longtemps dissimulée. Les Guyanes exhollandaise et ex-britannique, aujourd'hui indépendantes exercent un attrait sur la Guyane sous domination française. Les Antilles sous domination britannique [195] accèdent à l'indépendance. Castro à Cuba donne un nouveau visage aux Caraïbes. Oui la question est posée.

Dans l'immédiat, les forces françaises et leurs alliées, les hommes politiques actuels, députés et sénateurs, briseront sans nul doute cette première manifestation de l'esprit national martiniquais. Mais nous savons maintenant que les Guadeloupéens, les Martiniquais et les Guyanais seront indépendants et construiront leurs pays respectifs comme ils l'entendent. Le peuple algérien assure les Antillais et les Guyanais de sa fraternelle sympathie et les encourage à aiguiser leur combativité. Les Antillais et les Guyanais soldats, sous-officiers et officiers qui luttent contre leurs frères algériens pendant que les troupes françaises mitraillent leurs peuples à Fort-de-France ou à Basse Terre doivent refuser de se battre et déserter.

On sait maintenant que des liens existent entre la guerre d'Algérie et les récents événements qui ont ensanglanté la Martinique. Ce sont d'anciens fonctionnaires français d'Afrique du Nord, les expulsés du Maroc, de Tunisie et ceux qui étaient trop compromis en Algérie qui ont provoqué la riposte des masses martiniquaises. La brutale réaction du peuple martiniquais indique simplement que l'heure est venue de clarifier les problèmes et de dissiper les malentendus. 


\author{
Pour la révolution africaine \\ Écrits politiques
}

Quatrième partie. Vers la libération de l'Afrique.

\title{
Chapitre 21
}

\section{Unité et solidarité effective sont les conditions de la libération africaine 36}

\section{Retour à la table des matières}

Pour un observateur qui suit l'évolution du continent africain depuis deux ans, une conclusion s'impose avec une particulière évidence: progressivement les peuples dépendants sur qui pèse une domination étrangère accèdent à la souveraineté nationale.

Après le Ghana, la Guinée, voici que le Cameroun sous mandat français, le Togo, la Somalie sous mandat italien, la Nigéria deviennent indépendants. Les remous qui agitent la récente communauté française, les revendications franches, ou parfois voilées des dirigeants des différents pays de la communauté ne permettent aucun doute. Un processus est engagé, irréversible pourrait-on dire, si l'on faisait confiance aux formules stéréotypées. 


\section{La main de l'histoire, c'est la main de l'homme}

Les observateurs non étrangers se contentent donc de l'espoir généralisé dans le développement historique de ce que l'on a appelé le processus objectif de décolonisation, tandis que plus ou moins implicitement, on demande aux Africains de faire confiance à la bonne volonté des anciens maîtres et de ne [198] pas désespérer en tout cas des nécessités historiques qui rythment la reconversion de l'oppression coloniale.

Il est rigoureusement vrai que la décolonisation s'effectue, mais il est rigoureusement faux de prétendre et de croire que cette décolonisation soit le fruit d'une dialectique objective qui prend plus ou moins rapidement les apparences d'un mécanisme absolument inacceptable.

L'optimisme qui règne aujourd'hui en Afrique n'est pas un optimisme né du spectacle de forces de la nature enfin bénéfiques aux Africains. Cet optimisme n'est pas non plus dû à la constatation chez l'ancien oppresseur de dispositions moins inhumaines et plus bienveillantes. L'optimisme en Afrique est le produit direct de l'action révolutionnaire, politique ou armée - souvent les deux à la fois - des masses africaines.

On comprend maintenant pourquoi chaque nationaliste africain a une telle hantise de donner constamment une dimension africaine à son action. C'est que la lutte pour la liberté et l'indépendance nationale est dialectiquement liée à la lutte contre le colonialisme en Afrique.

L'ennemi de l'Africain sous domination française, ce n'est pas le colonialisme en tant qu'il s'exerce dans les limites strictes de sa nation, mais ce sont les formes du colonialisme, ce sont les manifestations du colonialisme, quels que soient le pavillon sous lequel il s'exerce et sévit.

Une grande partie de l'humanité a récemment tremblé dans ses assises les plus profondes devant le déferlement d'une idéologie: le nazisme qui fit ressurgir les méthodes de torture et de génocide des temps les plus reculés. 
Les pays les plus immédiatement visés par les manifestations du nazisme se liguèrent et prirent l'engagement, non pas seulement de libérer leur territoire occupé, mais de briser littéralement les reins au nazisme, d'extirper le mal là où il avait pris naissance, de liquider les régimes qu'il avait suscités.

Eh bien! les peuples africains doivent pareillement se souvenir qu'ils ont été confrontés à une forme de nazisme, à une forme d'exploitation de l'homme, de liquidation physique et spirituelle lucidement menée, que les manifestations française, anglaise, sud-africaine de ce mal là doivent les retenir, mais qu'ils doivent également s'engager à affronter ce mal en tant que mal sur l'ensemble du territoire africain.

Les pays européens sont aujourd'hui préoccupés par le problème [199] de la paix. Après s'être armés à outrance les blocs de l'Est et de l'Ouest s'aperçoivent avec effroi que tout nouveau conflit mondial mettrait en question l'existence même de la vie sur terre. Aussi une confrontation pacifique des deux conceptions du monde devient-elle indispensable.

C'est dans cette perspective que le général Eisenhower a entrepris son dernier périple, que le conseil de l'O.T.A.N. s'est réuni à Paris et qu'une confrontation au sommet est fixée pour les premiers mois de 1960.

Nous Africains disons que le problème de la paix entre les hommes, en l'occurrence non-africains, est fondamental, mais disons aussi que la libération de l'Afrique, des derniers bastions du colonialisme constitue le premier problème.

Quand nous Africains, nous disons que nous sommes neutres sur les rapports Est-Ouest, nous signifions que pour l'instant la seule question qui nous préoccupe est celle de notre combat contre le colonialisme. Cela veut dire que nous ne sommes absolument pas neutres devant le génocide mené par la France en Algérie ou devant l'apartheid en Afrique du Sud.

Notre neutralité signifie que nous n'avons pas à prendre position pour ou contre I'O.T.A.N., pour ou contre le pacte de Varsovie.

Dans le cadre de notre combat anti-colonialiste, nous ne tenons compte que de la fermeté de notre engagement et des appuis que nous 
accorde tel ou tel pays. Et dans ce cadre, nous pouvons dire que les peuples groupés sous le terme de pays de l'Est nous soutiennent de manière très ferme et que les pays dits de l'Ouest multiplient les ambiguiités.

\section{Des armes et des hommes}

Les peuples africains sont concrètement engagés dans une lutte globale contre le colonialisme et nous, Algériens, ne dissocions pas le combat que nous menons de celui des Rhodésiens ou des Kenyans. Notre solidarité à l'égard de nos frères Africains n'est pas verbale. Elle ne se traduit pas par le vote, par l'acclamation dans une réunion internationale de résolutions ou de condamnations. Les pays colonialistes, quand ils étaient en danger et que le fascisme, le nazisme les submergeaient, donc quand leur liberté et leur indépendance étaient menacées, n'ont pas hésité à ponctionner dans les masses africaines [200] et à lancer contre les positions nazies une majorité de leurs «coloniaux ». Aujourd'hui, ce sont la liberté et l'indépendance nationale des Africains qui se trouvent en question.

La solidarité interafricaine doit être une solidarité de fait, une solidarité d'action, une solidarité concrète en hommes, en matériel, en argent.

L'Afrique sera libre. Oui, mais il faut qu'elle se mette au travail, qu'elle ne perde pas de vue sa propre unité. C'est dans cet esprit qu'avait, entre autres, été, adopté l'un des points les plus importants du premier Congrès des peuples africains à Accra en 1958. Les peuples africains, était-il dit dans cette résolution, s'engagent à constituer une milice qui serait chargée d'appuyer les peuples africains en lutte pour leur indépendance.

Ce n'est pas un hasard si cette résolution a été escamotée par la presse occidentale. La violence des démocraties occidentales pendant leur guerre contre le nazisme, la violence des Etats-Unis d'Amérique à Hiroshima avec la bombe atomique, sans être un exemple, donnent une mesure de ce que les démocraties peuvent entreprendre quand leur vie est en danger. 
Nous Africains disons que depuis plus de 100 ans la vie de 200.000.000 d'Africains est une vie au rabais, contestée, une vie hantée perpétuellement par la mort. Nous disons que nous ne devrons pas faire confiance dans la bonne foi des colonialistes, mais que nous devons nous armer de fermeté et de combativité. L'Afrique ne sera pas libre par le développement mécanique des forces matérielles, mais c'est la main de l'Africain et son cerveau qui déclenchent et mèneront à bien la dialectique de la libération du continent.

À quelques jours de la $2^{e}$ Conférence des peuples africains qui doit se tenir aux portes de l'Algérie en feu et en sang, les Africains doivent se rappeler qu'il n'y a pas un optimisme objectif qui s'impose de manière plus ou moins mécanique, mais que l'optimisme doit être le sentiment qui accompagne l'engagement révolutionnaire et le combat.

Dans ces conditions, oui nous pouvons être optimistes. 
[201]

Pour la révolution africaine Écrits politiques

\section{Cinquième partie}

Unité africaine

Retour à la table des matières 
[203]

\author{
Pour la révolution africaine \\ Écrits politiques
}

Cinquième partie. Unité africaine.

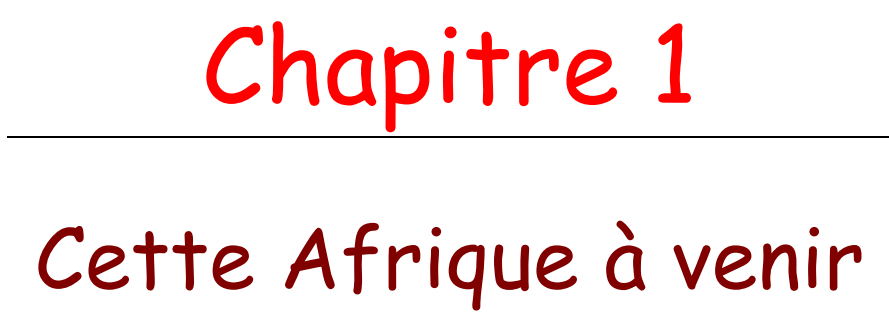

Retour à la table des matières

[À la fin de l'année 1958 les colonels de wilayas de l'A.L.N. ont tenu une réunion dans le Nord-Constantinois. Ils ont constaté à cette occasion le danger d'un étouffement progressif de la lutte armée à l'intérieur par suite du dispositif mis en place par l'ennemi (zones interdites, camps de regroupements tendant à couper l'A.L.N. de la population).

On décida d'envoyer à Tunis le colonel Amirouche (colonel de la wilaya III) pour expliquer la situation au G.P.R.A. et définir les moyens qui permettraient de ravitailler l'intérieur en armes, en munitions et en finances.

Le colonel Amirouche ne parvint jamais à rejoindre Tunis puisqu'il devait être tué par l'ennemi au cours de ce voyage dans la région de Bou-Saâda en mars 59.

C'est pour faire face à cette situation que le C.N.R.A. décida au cours de sa réunion de l'automne 1959 la création de l'Etat-Major. 
L'armée française ayant renforcé son dispositif aux frontières (ligne (halle) il devenait difficile de ravitailler l'intérieur par le Maroc et la Tunisie.

En mars 1960 Fanon était nommé à Accra. Pendant son séjour en Afrique occidentale il put constater qu'il existait une possibilité de renforcer la situation à l'intérieur à partir de la frontière sud c'est-àdire la frontière du Mali. Il prit également dans ce sens des contacts avec les responsables maliens et fit part de ses suggestions aux dirigeants algériens qui décidèrent la création d'une troisième base au sud du Sahara pour l'acheminement des armes vers les wilayas I et $V$.

Les notes qui suivent ont été écrites par Fanon au cours de la mission de reconnaissance et d'installation de cette base pendant l'été 1960.

$\grave{A}$ ce journal de bord sont jointes quelques indications techniques sous forme de notes hâtives et inachevées ou Fanon étudie les différentes solutions qui pourraient être adoptées sur le plan strictement opérationnel].

[204]

Mettre l'Afrique en branle, collaborer à son organisation, à son regroupement, derrière des principes révolutionnaires. Participer au mouvement ordonné d'un continent, c'était cela, en définitive, le travail que j'avais choisi. La première base de départ, le premier socle était représenté par la Guinée. Puis le Mali décidé à tout, fervent et brutal, cohérent et singulièrement acéré, étendait la tête de pont et ouvrait de précieuses perspectives. A l'Est Lumumba piétinait. Le Congo qui constituait la deuxième plage de débarquement des idées révolutionnaires, se trouvait pris dans un lacis pénible de contradictions stériles. Il fallait encore attendre avant d'investir efficacement les citadelles colonialistes qui s'appellent Angola, Mozambique, Kenya, Union SudAfricaine.

Tout était pourtant en place. Et voici que le système de défense colonialiste, quoique discordant, ranimait les vieux particularismes et émiettait la lave libératrice. Pour l'instant, il fallait donc tenir au Congo et avancer à l'Ouest. Pour nous Algériens, la situation était claire. Mais le terrain demeurait difficile, très difficile. A partir de l'Ouest, il nous fallait prouver par des manifestations concrètes que ce continent était un. Que derrière les options générales des dirigeants, 
il était possible de déterminer les points précis où les peuples, les hommes et les femmes, pouvaient se rencontrer, s'épauler, construire en commun. Le spectre de l'Occident, les teintes européennes étaient partout présents et actifs. Les zones française, anglaise, espagnole, portugaise demeuraient vivantes. Oxford s'opposait à la Sorbonne, Lisboa à Bruxelles, les patrons anglais aux patrons portugais, la livre au franc, l'Eglise catholique au protestantisme ou à l'Islam. Et au-dessus de tout cela, les Etats-Unis qui s'enfonçaient partout, dollars en tête, avec Armstrong comme héraut et les diplomates noirs américains, les bourses, les émissaires de la voix de l'Amérique... Et n'oublions pas l'Allemagne travailleuse, Israël défrichant le désert...

Travail difficile. Heureusement, dans chaque coin, des bras nous font signe, des voix nous répondent, des mains nous empoignent. Ça colle.

Le bruit rapide et tranquillisant des cités libérées qui rompent leurs amarres et s'avancent grandiloquentes mais nullement grandioses, ces anciens militants aujourd'hui admis définitivement [205] à tous leurs examens qui s'asseyent et... se souviennent, mais le soleil est encore très haut dans le ciel et si l'on écoute l'oreille collée au sol rouge, on entend très distinctement des bruits de chaînes rouillées, des « han » de détresse et les épaules vous en tombent tant est toujours présente la chair meurtrie dans ce midi assommant. L'Afrique de tous les jours, oh! pas celle des poètes, pas celle qui endort, mais celle qui empêche de dormir, car le peuple est impatient de faire, de jouer, de dire. Le peuple qui dit: Je veux me construire en tant que peuple, je veux bâtir, aimer, respecter, créer. Ce peuple qui pleure quand vous dites: je viens d'un pays où les femmes sont sans enfants et les enfants sans mères et qui chante: l'Algérie, pays frère, pays qui appelle, pays qui espère.

C'est bien l'Afrique, cette Afrique-là qu'il nous fallait lâcher dans le sillon continental, dans la direction continentale. Cette Afrique-là qu'il fallait orienter, mobiliser, lancer à l'offensive. Cette Afrique à venir.

L'Ouest. Conakry, Bamako. Deux villes mortes en surfaces, mais endessous, la température est insupportable pour ceux qui calculent, qui manoeuvrent, qui s'installent. A Conakry et à Bamako des hommes et des femmes frappent l'Afrique, la forgent avec amour et enthousiasme. 
Moumié. Le 30 septembre, nous nous sommes rencontrés à l'aérodrome d'Accra. Il allait à Genève pour de très importants entretiens. Dans trois mois me disait-il, on assistera à un reflux en masse du colonialisme au Cameroun.

$\grave{A}$ Tripoli, un brouillard interdisait tout atterrissage et trois heures durant, l'avion tourna au-dessus de l'aérodrome. Coûte que coûte, le pilote voulait atterrir. La tour de contrôle refusait la permission sollicitée mais le pilote courageux et inconscient avait décidé de poser ses dizaines de milliers de tonnes. «Ces gars-là jouent avec la vie des gens » me dit Félix.

C'était vrai. Mais nous, ne jouions-nous pas également avec la nôtre? Qu'était cette intrépidité du pilote en comparaison de nos vies perpétuellement en suspens? Aujourd'hui, Félix est mort. À Rome, quinze jours après, on devait se retrouver. Il était absent. Son père debout à l'arrivée d'Accra me voyait venir, seul, et une grande tristesse s'installait sur son visage.

Deux jours après une dépêche nous apprenait que Félix [206] était hospitalisé. Puis qu'on soupçonnait un empoisonnement. Kingué, viceprésident de I'U.P.C. et Marthe Moumié décidaient de se rendre à Genève. Quelques jours après la nouvelle nous parvenait: Félix Moumié était mort.

Nous n'avons guère senti cette mort. Un assassinat, mais exangue. Il n'y a eu ni rafales de mitraillettes, ni bombes. Empoisonnement au thallium. Ça n'a pas de sens. Thallium! Comment saisir cette cause? Une mort abstraite frappant l'homme le plus concret, le plus vivant, le plus impétueux. Le ton de Félix était constamment haut. Agressif, violent, coléreux, amoureux de son pays, haineux pour les lâches et les manœuvriers. Austère, dur, incorruptible. De l'essence révolutionnaire prise dans $60 \mathrm{kgs}$ de muscles et d'os.

Le soir, nous sommes allés réconforter les camarades du Cameroun. Le père, le visage buriné, impassible, inexpressif, m'écoutait lui parler de son fils. Et progressivement, le père cédait la place au militant. Oui, disait-il, le programme est net. Il faut coller au programme. Le père de Moumié, en cet instant, me rappelait ces parents d'Algérie qui écoutent dans une sorte d'hébétude le récit de la mort de leurs enfants. Qui de temps à autre interrogent, réclament une précision puis retom- 
bent dans cette inertie de communion qui semble les happer vers où, croient-ils, leurs fils se sont rendus.

L'action est là pourtant. Demain, tout à l'heure, il faudra porter la guerre chez l'ennemi, ne lui laisser aucun repos, le talonner, lui couper la respiration.

Partons. Notre mission: ouvrir le front Sud. De Bamako acheminer armes et munitions. Soulever la population saharienne, s'infiltrer jusqu'aux hauts plateaux algériens. Après avoir porté l'Algérie aux quatre coins d'Afrique, remonter avec toute l'Afrique vers I'Algérie africaine, vers le Nord, vers Alger, ville continentale. Ce que je voudrais: de grandes lignes, de grands canaux de navigations à travers le désert. Abrutir le désert, le nier, rassembler l'Afrique, créer le continent. Que du Mali s'engouffrent sur notre territoire des Maliens, des Sénégalais, des Guinéens, des Ivoiriens, des Ghanéens. Et ceux du Nigéria, du Togo. Que tous grimpent les pentes du désert et déferlent sur le bastion colonialiste. Prendre l'absurde et l'impossible à rebrousse-poil et lancer un continent à l'assaut des derniers remparts de la puissance coloniale.

[207]

Nous sommes huit: un commando; l'Armée, les transmissions, les commissaires politiques, le corps sanitaire. Chacun des couples doit prospecter selon sa propre discipline les possibilités de travail. Faire vite. Le temps presse. L'ennemi est encore tenace. En réalité, il ne croit pas à la défaite militaire. Mais moi je ne l'ai jamais sentie aussi possible, aussi à portée de pas. Il suffit de marcher, de foncer. Il ne s'agit même pas de stratégie. Nous avons des cohortes mobilisées et furieuses, amoureuses de notre combat, ardentes au travail. Nous avons l'Afrique avec nous. Mais qui s'en préoccupe. Un continent va se mettre en branle et l'Europe est langoureusement endormie. Il y a 15 ans, c'était l'Asie qui s'ébrouait. Alors on s'amusait. Aujourd'hui, l'Europe et les Etats-Unis se hérissent. Les 650 millions de Chinois, tranquilles possesseurs d'un secret immense, bâtissent à eux seuls un monde. Accouchement d'un monde.

Chawki. Drôle d'homme. Commandant de l'A.L.N. originaire du Souf. Petit, sec, les yeux implacables comme les ont en général les vieux maquisards. Depuis longtemps j'identifie l'ancienneté d'un maquisards au 
brillant de ses yeux. Ces yeux ne trompent pas. Ils disent ouvertement qu'ils ont assisté à des choses dures : répressions, tortures, canonnades, poursuites, liquidations... On note dans ces yeux une sorte de hauteur, de dureté quasi meurtrière. D'inintimidation aussi. Avec de pareils hommes on prend assez vite l'habitude de faire attention. Tout peut leur être dit mais il leur faut sentir et toucher la Révolution dans les paroles prononcées. Très difficile de les tromper, de les contourner, de les noyauter.

Pour l'instant Chawki et moi partageons le même lit. Nos discussions se prolongent assez tard dans la nuit et je suis en perpétuel émerveillement devant l'intelligence et la netteté de ses pensées. Diplômé de I'Université islamique de la Zitouna en Tunisie il a voulu entrer en contact avec la civilisation occidentale. Il s'est installé à Alger pour y apprendre le français, voir, juger, discriminer. Mais l'atmosphère d'Alger avec les colons méprisants, son ignorance totale de la langue française, l'hermétisme du milieu européen le décident à se rendre en France. Pendant deux ans il vit à Paris, s'introduit dans les cercles européens, hante les bibliothèques et dévore des centaines de livres.

[208]

Enfin il rentre en Algérie et se propose de mettre en valeur les terres de son père. 1954. Il décroche son fusil de chasse et rejoint les frères. Il connaît à merveille le Sahara. Cette immensité désertique et inhumaine revêt quand il en parle une infinité de détails. Coins hospitaliers, voies dangereuses, régions mortelles, directions de pénétration, le Sahara est un véritable monde ou Chawki évolue avec une témérité et une perspicacité de grand stratège. Les Français ne se doutent pas des tours que cet homme s'apprête à leur jouer.

Il faut dire que notre mission a failli se terminer dans les salles d'interrogatoire d'Algérie. D'Accra l'employé de Ghana Airways, Mensah qui exige à chaque réservation quelques dizaines de milliers de francs nous avait confirmé le trajet Monrovia-Conakry. Or à l'aérodrome libérien on nous apprit que l'avion était boudé et qu'il nous fallait attendre le lendemain pour rejoindre Conakry par un avion d'Air France. Les employés furent anormalement prévenants avec nous et nous proposèrent de prendre à la charge de la Compagnie tous les frais de séjour. Cette exemplaire sollicitude, la nationalité française de plu- 
sieurs employés et l'allure de cantinière d'une française empressée et on ne peut plus endormante nous amenèrent à changer de route. Nous décidâmes de quitter Monrovia par la route et d'entrer en Guinée la nuit par N'Zérékoré.

Jusqu'à la dernière minute les employés furent persuadés que nous prenions l'avion qui ce jour là avait deux heures de retard.

Le deuxième bureau français avait bel et bien pris l'affaire en mains. L'avion en quittant Robertsfield au lieu de se diriger sur Freetown rebroussa chemin et atterrit à Abidjan où il fut fouillé par les forces françaises.

Il est clair que le gouvernement ivoirien a une responsabilité de premier plan dans cette affaire. Une telle opération n'aurait pas pu se dérouler sans sa complicité ou du moins sa bienveillance. HouphouëtBoigny que certains tentent de blanchir continue à jouer dans le système colonial français un rôle de premier plan et les peuples africains gagneraient à l'isoler et à précipiter sa chute. Houphouët-Boigny est objectivement le frein le plus conscient à l'évolution et à la libération de l'Afrique. Enfin le deuxième bureau a dû se mordre les doigts. Pareille opération n'est rentable que si elle réussit.

[209]

L'échec public dans ces conditions révèle des méthodes de brigandage qui risquent de provoquer un raidissement chez ceux-là mêmes qui avaient accepté de fermer les yeux.

J'espère en tout cas que les services français ont perdu notre trace.

Nous voici à Bamako capitale du Mali. Modibo Keita toujours militant comprend rapidement. Pas besoin de grands discours. Nos séances de travail vont vite. Très rapidement les frères des transmissions discutent avec lui et décident d'installer à Kayes un service d'écoute. Je pense que d'ici le 5 décembre il commencera à fonctionner. Pour le moment nous sommes logés au centre d'accueil de la caserne de Bamako. Branle-bas de combat tous ces jours. N'Krumah arrive le 21 en visite officielle.

À Bamako l'élément français est encore important. Librairies, pharmacies, maisons de commerce appartiennent en majorité aux co- 
lons français. Çà et là on croise un commandant, deux sergents... Hier dimanche 20 un adjudant français servant dans l'Armée Malienne venant de Ségou avec une compagnie est arrivé au centre d'accueil. Très poliment il s'est présenté et nous a serré la main. Il voulait savoir si nous ne pouvions pas mettre un lit à sa disposition. Il faut un peu d'humour dans tout cela. En tout cas nous avons fait placer une sentinelle armée à partir de 20 heures. De temps à autre des voitures pilotées par des Européens croisent autour de la villa. Pas très sûr, ce coin. Heureusement les choses se précipitent. Mardi 22 à 5 heures du matin nous partons pour Gao. La route Bamako-Tombouctou est impraticable.

De Bamako nous atteignons Segou où Jouanelle nous accueille. Plein d'essence et nous atteignons San. Puis Mopti. A Mopti une anicroche. A la sortie de la ville: un barrage de gendarmerie, et les sentinelles exigent nos passeports. Discussions pénibles car malgré le papier du Ministre de l'Intérieur les gendarmes veulent connaître nos identités. Enfin le Chef de poste arrive et force m'est de me présenter. Mais il semble que nous soyions en présence d'un homme qui cherche le renseignement. Il veut savoir la nature de la mission et les qualités de ceux qui m'accompagnent.

Pour le coup je me mets en colère et lui demande de me retenir et de marrêter pour refus d'exhiber les papiers. Placé [210] dans cette situation il comprend qu'il a gaffé et nous laisse partir non sans avoir promis le secret absolu.

La route Mopti-Douentza est une galéjade. Au milieu d'une forêt on suit en devinant les traces d'une voiture qui a dû passer par là il y a 6 mois. Une telle exploration en pleine nuit est très pénible et plus d'une fois nous nous perdons. Enfin à 2 heures du matin nous arrivons. Personne dans le village. Le commandant est absent et sa femme nous envoie au campement... qui est fermé. Quelques-uns dans la voiture, d'autres ailleurs, on arrive tout de même à prendre un peu de repos. $A$ 7 heures, départ sur Gao via Hombori. A 21 heures nous frappons chez le commandant. Dix minutes plus tard nous sommes en plein travail. L'affaire se présente bien et les Maliens sont absolument décidés à nous aider dans la création de ce $3^{e}$ front. On a dans le temps parlé de l'odyssée Leclerc. Celle que nous préparons, si le gouvernement français ne comprend pas à temps, transformera l'épisode Leclerc en une banale excursion. A Gao nous trouvons une documentation complète 
laissée par les services secrets français sur les confins algéromarocains. Tous les noms d'Algériens y vivant sont mentionnés. Egalement sont mentionnés en marge leur plus ou moins grande bienveillance à l'égard des idées nationalistes. Facilement nous trouvons en négatif l'ossature d'une cellule de travail et de passage. Merci commandant Cardaire.

Après deux jours à Gao nous nous dirigeons sur Aguerhoc. Le commandant de Gao nous fait abandonner nos vêtements peuhls et nous offre à chacun une bonne tenue de goumier avec un Mas 36 et 20 cartouches. On aura l'occasion d'ailleurs de tuer une outarde et plusieurs biches.

À Aguerhoc nous rencontrons vers 23 heures le chef de la subdivision de Kidal qui est en compagnie du Chef de poste de Tessalit. Présentation. Trente minutes plus tard nous discutons stratégie, terrain, passage...

C'est palpitant de vivre ces instants. Il a fallu que ces deux responsables sachent qui nous étions pour que toute une immense complicité, latente jusqu'ici, s'exprime. Ce que nous voulons, ils nous le donnent. Nous voulons voir de près la frontière, Tessalit, Bouressa en face de Tir Zaouaten où les Français pris de court aménagent un aérodrome...? D'accord.

[211]

Et nous voilà partis sur près de $1000 \mathrm{kms}$ de piste. Cette partie du Sahara n'est point monotone. Même le ciel là-haut change constamment. Il y a quelques jours nous avons assisté à un coucher de soleil qui faisait violette la robe du ciel. Aujourd'hui c'est un rouge très dur qui limite le regard. Aguerhoc, Tessalit, Bouressa. À Tessalit nous traversons le camp militaire français. Un militaire français buste nu nous fait des signes amicaux. Ses bras lui tomberaient s'il pouvait deviner qui se dissimule sous ces tenues de goumiers.

À Bouressa nous prenons contact avec un groupe nomade malien. Nous apprenons de plus en plus de détails sur les forces françaises. Bordj le Prieur, Tir Zaouaten, Bidon V.

Et, au-delà, Tamanrasset où par des recoupements nous arrivons à nous faire une idée assez exacte des effectifs français. Les guides 
que nous trouvons à Bouressa ont l'air sérieux et décidés. Il faudra les utiliser en priorité.

$\grave{A}$ Kidal je me jette sur quelques bouquins d'histoire du Soudan. Je revis avec l'intensité que confèrent les circonstances et les lieux les vieux empires du Ghana, du Mali, de Gao et l'impressionnante odyssée des troupes marocaines avec le fameux Djouder. Tout n'est pas simple. Voici que l'Algérie en guerre vient solliciter l'aide du Mali. Or pendant ce temps le Maroc exige la Mauritanie et une partie du Mali... Egalement une partie de l'Algérie.

Cette région saharienne travaillée par tant d'influences et où les officiers français ne cessent de créer des noyaux de dissidence voici que nous nous préparons à la remuer de fond en comble autour d'un champ de bataille où il faudra beaucoup de rigueur et de sang-froid. Quelques réflexions recueillies çà et là, l'accent toujours appuyé dès qu'il est question de l'Islam et de la race exigent une prudence redoublée.

Le colonialisme et ses dérivés ne constituent pas à vrai dire les ennemis actuels de l'Afrique. A brève échéance ce continent sera libéré. Pour ma part plus je pénètre les cultures et les cercles politiques plus la certitude s'impose à moi que le grand danger qui menace l'Afrique est l'absence d'idéologie. La vieille Europe a peiné des siècles avant de parfaire l'unité nationale des Etats. Et lors même qu'un point final pouvait y être mis, que de guerres encore! Avec le triomphe du socialisme en Europe orientale on assiste à une disparition [212] spectaculaire des vieilles rivalités, des traditionnelles revendications territoriales. $\mathrm{Ce}$ foyer de guerres et d'assassinats politiques que représentaient la Bulgarie, la Hongrie, l'Estonie, la Slovaquie, l'Albanie ont fait place à un monde cohérent dont l'objectif est l'édification de la société socialiste.

En Afrique par contre les pays qui accèdent à l'indépendance sont aussi instables que leurs neuves bourgeoisies ou leurs princes rénovés. Après quelques pas hésitants dans l'arène internationale, les bourgeoisies nationales ne sentant plus la menace de la puissance coloniale traditionnelle se découvrent soudain de grands appétits. Et comme elles n'ont pas encore la pratique politique elles entendent mener cette affaire comme leur négoce. Prébendes, menaces, voire littéralement dé- 
pouillement de la victime. Tout cela est évidemment regrettable car les petits Etats n'ont d'autre ressource que de supplier l'ancienne Métropole de demeurer encore un peu. Egalement dans ces pseudo-Etats impérialistes une politique militariste à outrance entraîne la diminution des investissements publics dans des pays encore par endroits moyenâgeux. Les ouvriers mécontents subissent une répression aussi impitoyable que celle des périodes coloniales. Syndicats et partis politiques d'opposition sont confinés dans une quasi-clandestinité. Le peuple, le peuple qui avait tout donné aux heures difficiles de la lutte de libération nationale s'interroge mains et ventre vides sur le degré de réalité, de sa victoire.

Depuis près de trois ans j'essaie de faire sortir la fumeuse idée d'Unité Africaine des marasmes subjectivistes voire carrément fantasmatiques de la majorité de ses supporters. L'Unité Africaine est un principe à partir duquel on se propose de réaliser les Etats-Unis d'Afrique sans passer par la phase nationale chauvine bourgeoise avec son cortège de guerres et de deuils.

Pour amorcer cette Unité toutes les combinaisons sont possibles.

Certains comme la Guinée, le Ghana, le Mali, et demain peut-être l'Algérie mettent au premier plan l'action politique. D'autres comme le Libéria et le Nigéria insistent sur la coopération économique. La R.A.U. de son côté insisterait [213] davantage sur le plan culturel. Tout est possible et les uns et les autres devraient éviter de discréditer ou de dénoncer ceux qui voient cette unité, ce rapprochement des Etats africains d'une façon qui diffère de la leur. Ce qu'il faut éviter c'est la tension ghanéo-sénégalaise, la tension somalo-éthiopienne, marocomauritanienne, congolo-congolaise... En réalité les Etats colonisés qui ont accédé, à l'indépendance par la voie politique semblent n'avoir d'autres préoccupations que de se trouver un vrai champ de bataille avec des blessures et des destructions. Il est clair toutefois que cette explication psychologique, qui fait appel à un hypothétique besoin de défoulement de l'agressivité, ne nous satisfait pas. Il nous faut encore une fois revenir aux schémas marxistes. Les bourgeoisies triomphantes sont les plus impétueuses, les plus entreprenantes, les plus annexionnistes qui soient (ce n'est pas pour rien que la bourgeoisie française de 1789 mit l'Europe à feu et à sang). 


\section{Indications techniques}

$1^{\circ}$ Passages par camions: difficile à réaliser dans l'immédiat. Il faut préparer la chose. Contacter le chauffeur. Puis étudier le processus. Etudier les dépôts. Demandera, si l'on veut s'entourer du minimum de précaution et s'assurer le maximum de succès, au moins 3 mois de préparation à partir du début réel de l'application du projet.

$2^{\circ}$ Tout le problème est de savoir ce que l'on veut :

a) ou alimenter des forces déjà existantes au Sahara

b) ou alimenter les wilayas $I, V$ et les restes de la VI

c) ou créer littéralement une série de lignes d'attaque perpendiculaires à l'atlas tellien qui éventuellement rencontreraient et travailleraient de concert avec les wilayas déjà existantes. Evidemment on peut répondre que ces choix ne sont pas exclusifs et que ces trois options peuvent se contenir dans un même programme. De toutes façons il faut une priorité à l'une de ces trois possibilités même si l'ensemble de l'opération saharienne devait les contenir toutes les trois. Personnellement je penche vers le point $c$.

comment le réaliser?

Avant toutes choses porter sur la frontière le maximum de matériel. Dans les 2 mois qui viennent:

$\begin{array}{ll}10.000 & \text { fusils : } \\ 4.000 & \text { P.M. : } \\ 1.500 & \text { F.M. : } \\ 600 & \text { mitrailleuses : } \\ 3 \text { à } 400 & \text { lance-rockets. }\end{array}$


Les mines et les grenades non directement utilisables au Sahara devraient être réservées à l'alimentation des wilayas du Nord.

Que faire de ces armes c'est-à-dire comment réaliser l'action?

Je vois la chose selon deux directions :

l'une verticale:

l'autre horizontale.

La direction horizontale est la direction d'implantation tandis que la verticale est de pénétration.

Une quarantaine d'éléments connaissant le Sahara et excellents militants pourraient être nommés chefs de commando.

Ces commando évolueraient par tranches de 10. Chaque commando pourrait comporter au départ 20 à 25 membres, à charge pour les chefs d'en porter rapidement le nombre à 100 , même 150 . Le recrutement se ferait au départ localement. Soit éléments algériens résidant au Mali soit carrément éléments touareg maliens. Cela peut se faire en un mois et demi. D'ici le 15 janvier on peut armer et introduire en Algérie 500 à 800 hommes armés.

La première vague est de politisation, de mobilisation. Elle devra éviter l'accrochage et laisser passer les occasions de frapper l'ennemi, même si le succès est assuré. Son rôle est de réveiller les populations, de les rassurer sur l'avenir, de montrer l'armement de l'A.L.N., de les détacher psychologiquement et mentalement de l'emprise ennemie.

Dans chaque tribu importante rencontrée, le commando doit recruter 3 à 4 nouveaux membres et laisser 3 ou 4 de ses membres d'origine. Pourquoi :

a) les nouvelles recrues connaissent le terrain au-delà et servent au début de trait-d'union, d'interprètes politiques avec les tribus du Nord: 
b) les membres du commando laissés sur place préparent [215] les différents canaux de liaisons qui accueilleront les vagues suivantes.

On aurait donc alors le schéma suivant :

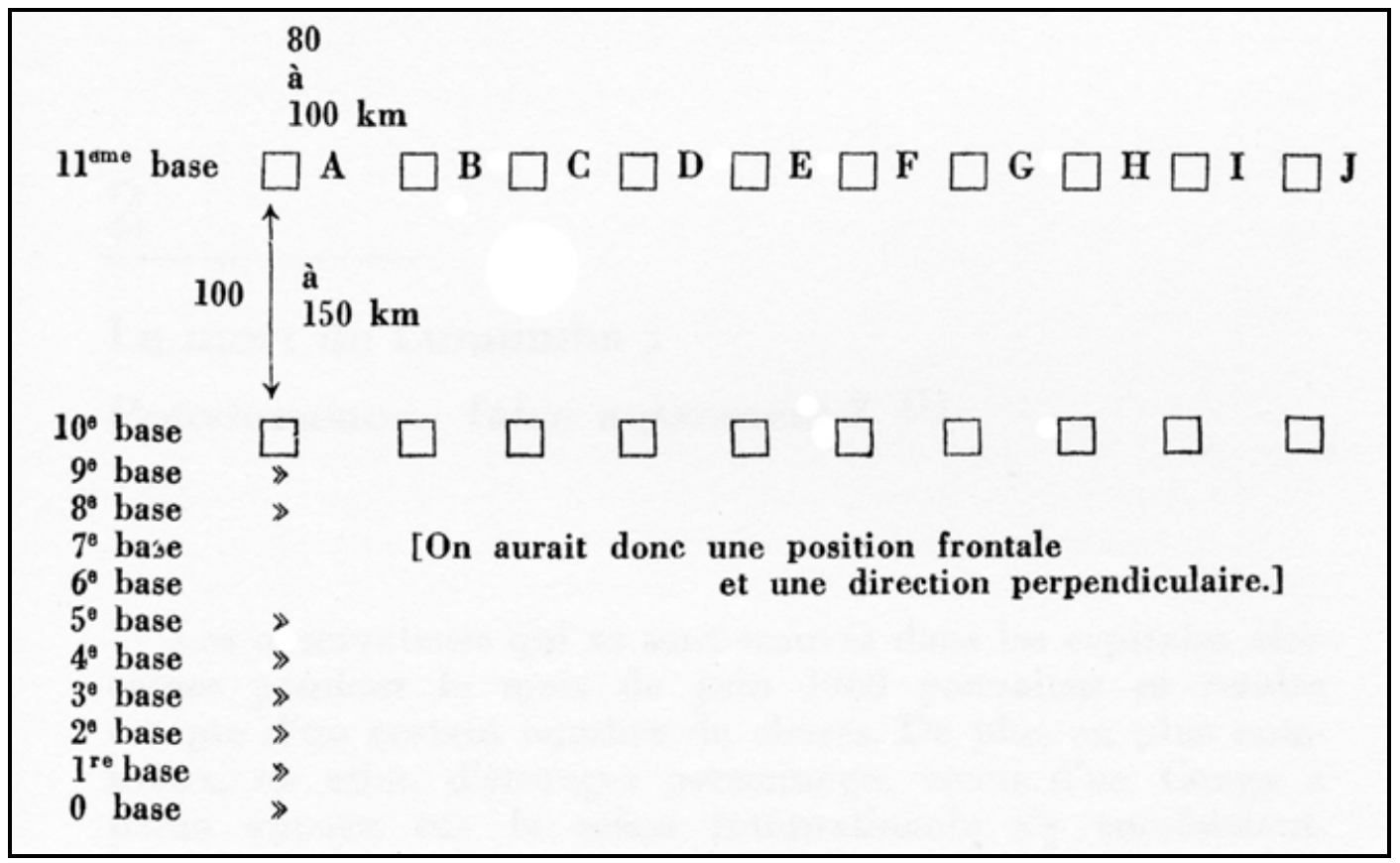

Dans le même temps des colonnes de ravitaillement monteraient en base 1.

La base 2 enverrait en base 1 des colonnes de ravitaillement.

La base 3 en base 2... ainsi de suite. Ce n'est que lorsque les bases avancées auront reçu 3 ou 4 stocks de ravitaillement que pourra se poser la question du déclenchement des opérations.

$\grave{A}$ ce moment d'ailleurs les contacts avec les routiers et peut-être une situation meilleure au Fezzan nous permettront d'alimenter régulièrement les groupes de l'A.L.N. 
Chaque groupe de 25 aurait l'armement suivant :

2 lance-rockets et 20 obus :

2 mitrailleuses dont 1 anti-aérienne: 3 F.M.

Les groupes partiraient à 2 jours d'intervalle. Il faudrait prévoir 1 poste de radio au départ pour la base 0 qui se trouverait en $D$

[216]

pour la $4 e$ base qui se trouverait en $\mathrm{J}$ pour la $9 e$ base qui se trouverait en $A$ et 2 ou 3 postes le long de la frontière.

Ces postes frontière auront des heures d'écoute avec l'État-Major Nord et chacun des postes de base 0,4 et 9 . 
Pour la révolution africaine

Écrits politiques

Cinquième partie. Unité africaine.

\section{Chapitre 2}

\section{La mort de Lumumba: \\ Pouvions-nous faire autrement? ${ }_{37}$}

\section{Retour à la table des matières}

Les observateurs qui se sont trouvés dans les capitales africaines pendant le mois de juin 1960 pouvaient se rendre compte d'un certain nombre de choses. De plus en plus nombreux, en effet, d'étranges personnages venus d'un Congo à peine apparu sur la scène internationale s'y succédaient. Que disaient ces Congolais? Ils disaient n'importe quoi. Que Lumumba était vendu aux Ghanéens. Que Gizenga était acheté par les Guinéens, Kashamura par les Yougoslaves. Que les civilisateurs belges partaient trop tôt, etc...

Mais si l'on s'avisait d'attraper dans un coin un de ces Congolais, de l'interroger, alors on s'apercevait que quelque chose de très grave se tramait contre l'indépendance du Congo et contre l'Afrique.

37 Afrique Action, №19, 20 février 1961. 
Des sénateurs, des députés congolais aussitôt après les fêtes de l'indépendance se sauvaient hors du Congo et se rendaient... aux EtatsUnis. D'autres s'installaient pour plusieurs semaines à Brazzaville. Des syndicalistes étaient invités à New-York. Là encore, si l'on prenait l'un de ces députés ou de ces sénateurs dans un coin et qu'on l'interrogeait, il devenait patent que tout un processus très précis allait se mettre en route.

[218]

Dès avant le $1^{\text {er }}$ juillet 1960, l'opération Katanga était lancée. Son but? Bien sûr, sauvegarder l'Union Minière. Mais au-delà de cette opération, c'est une conception belge qui était défendue. Un Congo unifié, avec un gouvernement central, allait à l'encontre des intérêts belges. Appuyer les revendications décentralisatrices des diverses provinces, susciter ces revendications, les alimenter, telle était la politique belge avant l'indépendance.

Dans leur tâche, les Belges étaient aidés par les autorités de la Fédération Rhodésies-Nyassaland. On sait aujourd'hui, et M. Hammarskjoeld mieux que quiconque, qu'avant le 30 juin 1960, un pont aérien Salisbury-Elizabethville alimentait le Katanga en armes. Lumumba avait certain jour proclamé que la libération du Congo serait la première phase de la complète indépendance de l'Afrique Centrale et Méridionale et il avait très précisément fixé ses prochains objectifs: soutien des mouvements nationalistes en Rhodésie, en Angola, en Afrique du Sud.

Un Congo unifié ayant à sa tête un anticolonialiste militant constituait un danger réel pour cette Afrique sudiste, très proprement sudiste, devant laquelle le reste du monde se voile la face. Nous voulons dire devant laquelle le reste du monde se contente de pleurer, comme à Sharpville, ou de réussir des exercices de style à l'occasion des journées anticolonialistes. Lumumba, parce qu'il était le chef du premier pays de cette région à obtenir l'indépendance, parce qu'il savait concrètement le poids du colonialisme, avait pris l'engagement au nom de son peuple de contribuer physiquement à la mort de cette Afriquelà. Que les autorités du Katanga et celles du Portugal aient tout mis en œuvre pour saboter l'indépendance du Congo ne nous étonne point. Qu'elles aient renforcé l'action des Belges et augmenté la poussée des forces centrifuges au Congo est un fait. Mais ce fait n'explique pas la 
détérioration qui s'est installée progressivement au Congo, ce fait n'explique pas l'assassinat froidement décidé, froidement mené de Lumumba, cette collaboration colonialiste au Congo est insuffisante à expliquer pourquoi en février 1961 l'Afrique va connaître autour du Congo sa première grande crise.

Sa première grande crise car il faudra qu'elle dise si [219] elle avance ou si elle recule. Il faudra qu'elle comprenne qu'il ne lui est plus possible d'avancer par régions, que, comme un grand corps qui refuse toute mutilation, il lui faudra avancer en totalité, qu'il n'y aura pas une Afrique qui se bat contre le colonialisme et une autre qui tente de s'arranger avec le colonialisme. Il faudra que l'Afrique, c'est-à-dire les Africains, comprennent qu'il n'y a jamais de grandeur à atermoyer et qu'il n'y a jamais de déshonneur à dire ce que l'on est et ce que l'on veut et qu'en réalité l'habileté du colonisé ne peut être en dernier ressort que son courage, la conception lucide de ses objectifs et de ses alliances, la ténacité qu'il apporte à sa libération.

Lumumba croyait en sa mission. Il avait une confiance exagérée dans le peuple. Ce peuple, pour lui, non seulement ne pouvait se tromper, mais ne pouvait être trompé. Et de fait, tout semblait lui donner raison. Chaque fois par exemple que dans une région les ennemis du Congo arrivaient à soulever contre lui l'opinion, il lui suffisait de paraître, d'expliquer, de dénoncer, pour que la situation redevienne normale. Il oubliait singulièrement qu'il ne pouvait être partout à la fois et que le miracle de l'explication était moins la vérité de ce qu'il exposait que la vérité de sa personne.

Lumumba avait perdu la bataille pour la présidence de la République. Mais parce qu'il incarnait d'abord la confiance que le peuple congolais avait mise en lui, parce que confusément les peuples africains avaient compris que lui seul était soucieux de la dignité de son pays, Lumumba n'en continua pas moins à exprimer le patriotisme congolais et le nationalisme africain dans ce qu'ils ont de plus rigoureux et de plus noble.

Alors d'autres pays beaucoup plus importants que la Belgique ou le Portugal décidèrent de s'intéresser directement à la question. Lumumba fut contacté, interrogé. Après soit périple aux États-Unis la décision était prise : Lumumba devait disparaître.

Pourquoi? Parce que les ennemis de l'Afrique ne s'y étaient pas trompés. Ils s'étaient parfaitement rendus compte que Lumumba était 
vendu, vendu à l'Afrique s'entend. C'est-à-dire qu'il n'était plus à acheter.

Les ennemis de l'Afrique se sont rendu compte avec un [220] certain effroi que si Lumumba réussissait, en plein cœur du dispositif colonialiste, avec une Afrique française se transformant en communauté rénovée, une Angola « province portugaise » et enfin l'Afrique orientale, c'en était fini de « leur » Afrique au sujet de laquelle ils avaient des plans très précis.

Le grand succès des ennemis de l'Afrique, c'est d'avoir compromis les Africains eux-mêmes. Il est vrai que ces Africains étaient directement intéressés par le meurtre de Lumumba. Chefs de gouvernements fantoches, au sein d'une indépendance fantoche, confrontés jour après jour à une opposition massive de leurs peuples, ils n'ont pas été longs à se convaincre que l'indépendance réelle du Congo les mettrait personnellement en danger.

Et il y eut d'autres Africains, un peu moins fantoches, mais qui s'effraient dès qu'il est question de désengager l'Afrique de l'Occident. On dirait que ces Chefs d'Etat africains ont toujours peur de se trouver en face de l'Afrique. Ceux-là aussi, moins activement, mais consciemment, ont contribué à la détérioration de la situation au Congo. Petit à petit, on se mettait d'accord en Occident qu'il fallait intervenir au Congo, qu'on ne pouvait pas laisser les choses évoluer à ce rythme.

Petit à petit, l'idée d'une intervention de l'O.N.U. prenait corps. Alors on peut dire aujourd'hui que deux erreurs simultanées ont été commises par les Africains.

Et d'abord par Lumumba quand il sollicita l'intervention de l'O.N.U. Il ne fallait pas faire appel à l'O.N.U. L'O.N.U. n'a jamais été capable de régler valablement un seul des problèmes posés à la conscience de l'homme par le colonialisme, et chaque fois qu'elle est intervenu, c'était pour venir concrètement au secours de la puissance colonialiste du pays oppresseur.

Voyez le Cameroun. De quelle paix jouissent les sujets de M. Ahidjo tenus en respect par un corps expéditionnaire français qui, la plupart du temps, a fait ses premières armes en Algérie? L'O.N.U. a cependant contrôlé l'autodétermination du Cameroun et le gouvernement français y a installé un « exécutif provisoire». 
Voyez le Viet-Nam.

[221]

Voyez le Laos.

Il n'est pas vrai de dire que l'O.N.U. échoue parce que les causes sont difficiles.

En réalité, I'O.N.U. est la carte juridique qu'utilisent les intérêts impérialistes quand la carte de la force brute a échoué.

Les partages, les commissions mixtes contrôlées, les mises sous tutelle sont des moyens légaux internationaux de torturer, de briser la volonté d'indépendance des peuples, de cultiver l'anarchie, le banditisme et la misère.

Car enfin, avant l'arrivée de l'O.N.U., il n'y avait pas de massacres au Congo. Après les bruits hallucinants propagés à dessein à l'occasion du départ des Belges, on ne comptait qu'une dizaine de morts. Mais depuis l'arrivée de l'O.N.U. on a pris l'habitude chaque matin d'apprendre que les Congolais par centaines s'entremassacraient.

On nous dit aujourd'hui que des provocations répétées furent montées par des Belges déguisés en soldats de l'Organisation des Nations Unies. On nous révèle aujourd'hui que des fonctionnaires civils de I'O.N.U. avaient en fait mis en place un nouveau gouvernement le troisième jour de l'investiture de Lumumba. Alors on comprend beaucoup mieux ce que l'on a appelé la violence, la rigidité, la susceptibilité de Lumumba.

Tout montre en fait que Lumumba fut anormalement calme.

Les chefs de mission de l'O.N.U. prenaient contact avec les ennemis de Lumumba et avec eux arrêtaient des décisions qui engageaient l'Etat du Congo. Comment un chef de gouvernement doit-il réagir dans ce cas? Le but recherché et atteint est le suivant: manifester l'absence d'autorité, prouver la carence de l'Etat.

Donc motiver la mise sous séquestre du Congo.

Le tort de Lumumba a été alors dans un premier temps de croire en l'impartialité amicale de l'O.N.U. Il oubliait singulièrement que l'O.N.U. dans l'état actuel n'est qu'une assemblée de réserve, mise sur pied par les Grands, pour continuer entre deux conflits armés la « lutte pacifique » pour le partage du monde. Si M. Iléo en août 1960 affirmait à qui 
voulait l'entendre qu'il fallait pendre Lumumba, si les membres du cabinet Lumumba ne savaient que faire des dollars [222] qui, à partir de cette époque, envahirent Léopoldville, enfin si Mobutu tous les soirs se rendait à Brazzaville pour y faire et $y$ entendre ce que l'on devine mieux aujourd'hui, pourquoi alors s'être tourné avec une telle sincérité, une telle absence de réserve vers l'O.N.U. ?

Les Africains devront se souvenir de cette leçon. Si une aide extérieure nous est nécessaire, appelons nos amis. Eux seuls peuvent réellement et totalement nous aider à réaliser nos objectifs parce que précisément, l'amitié qui nous lie à eux est une amitié de combat.

Mais les pays africains de leur côté, ont commis une faute en acceptant d'envoyer leurs troupes sous le couvert de l'O.N.U. En fait, ils admettaient d'être neutralisés et sans s'en douter, permettaient aux autres de travailler.

Il fallait bien sûr envoyer des troupes à Lumumba, mais pas dans le cadre de l'O.N.U. Directement. De pays ami à pays ami. Les troupes africaines au Congo ont essuyé une défaite morale historique. L'arme au pied, elles ont assisté sans réagir (parce que troupes de l'O.N.U.) à la désagrégation d'un Etat et d'une nation que l'Afrique entière avait pourtant salués et chantés. Une honte.

Notre tort à nous, Africains, est d'avoir oublié que l'ennemi ne recule jamais sincèrement. Il ne comprend jamais. Il capitule, mais ne se convertit pas.

Notre tort est d'avoir cru que l'ennemi avait perdu de sa combativité et de sa nocivité. Si Lumumba gêne, Lumumba disparaît. L'hésitation dans le meurtre n'a jamais caractérisé l'impérialisme.

Voyez Ben M'Hidi, voyez Moumié, voyez Lumumba. Notre tort est d'avoir été légèrement confus dans nos démarches. Il est de fait qu'en Afrique, aujourd'hui, les traîtres existent. Il fallait les dénoncer et les combattre. Que cela soit dur après le rêve magnifique d'une Afrique ramassée sur elle-même et soumise aux mêmes exigences d'indépendance véritable ne change rien à la réalité.

Des Africains ont cautionné la politique impérialiste au Congo, ont servi d'intermédiaires, ont cautionné les activités et les singuliers silences de I'O.N.U. au Congo. 
Aujourd'hui ils ont peur. Ils rivalisent de tartufferies au [223] tour de Lumumba déchiqueté. Ne nous y trompons point, ils expriment la peur de leurs mandants. Les impérialistes eux aussi ont peur. Et ils ont raison car beaucoup d'Africains, beaucoup d'Afro-Asiatiques ont compris. Les impérialistes vont marquer un temps d'arrêt. Ils vont attendre que "l'émotion légitime» se calme. Nous devons profiter de ce court répit pour abandonner nos craintives démarches et décider de sauver le Congo et l'Afrique.

Les impérialistes ont décidé d'abattre Lumumba. Ils l'ont fait. Ils ont décidé de constituer des légions de volontaires. Elles sont déjà sur place.

L'aviation katangaise sous les ordres de pilotes sud-africains et belges a commencé depuis plusieurs jours les mitraillages au sol. De Brazzaville, des avions étrangers se rendent bondés de volontaires et d'officiers parachutistes au secours d'un certain Congo.

Si nous décidons de soutenir Gizenga, nous devons le faire résolument.

Car nul ne connaît le nom du prochain Lumumba. Il y a en Afrique une certaine tendance représentée par certains hommes. C'est cette tendance dangereuse pour l'impérialisme qui est en cause. Gardonsnous de ne jamais l'oublier: c'est notre sort à tous qui se joue au Congo.

Fin du texte 